\title{
Bayesian Multivariate Time Series Methods for Empirical Macroeconomics*
}

\author{
Gary Koop \\ University of Strathclyde \\ Dimitris Korobilis \\ University of Strathclyde
}

April 2010

\begin{abstract}
Macroeconomic practitioners frequently work with multivariate time series models such as VARs, factor augmented VARs as well as timevarying parameter versions of these models (including variants with multivariate stochastic volatility). These models have a large number of parameters and, thus, over-parameterization problems may arise. Bayesian methods have become increasingly popular as a way of overcoming these problems. In this monograph, we discuss VARs, factor augmented VARs and time-varying parameter extensions and show how Bayesian inference proceeds. Apart from the simplest of VARs, Bayesian inference requires the use of Markov chain Monte Carlo methods developed for state space models and we describe these algorithms. The focus is on the empirical macroeconomist and we offer advice on how to use these models and methods in practice and include empirical illustrations. A website provides Matlab code for carrying out Bayesian inference in these models.
\end{abstract}

\section{Introduction}

The purpose of this monograph is to offer a survey of the Bayesian methods used with many of the models used in modern empirical macroeconomics. These models have been developed to address the fact that most questions of interest to empirical macroeconomists involve several variables and, thus, must be addressed using multivariate time series methods. Many different multivariate time series models have been used in macroeconomics, but since the pioneering work of Sims (1980), Vector Autoregressive (VAR) models have been among the most popular. It soon became apparent that, in many applications, the assumption that the VAR coefficients were constant over time might be a poor one. For instance, in practice, it is often found that the macroeconomy of the

*Both authors are Fellows at the Rimini Centre for Economic Analysis. Address for correspondence: Gary Koop, Department of Economics, University of Strathclyde, 130 Rottenrow, Glasgow G4 0GE, UK. Email: Gary.Koop@strath.ac.uk 
1960s and 1970s was different from the 1980s and 1990s. This led to an interest in models which allowed for time variation in the VAR coefficients and time-varying parameter VARs (TVP-VARs) arose. In addition, in the 1980s many industrialized economies experienced a reduction in the volatility of many macroeconomic variables. This Great Moderation of the business cycle led to an increasing focus on appropriate modelling of the error covariance matrix in multivariate time series models and this led to the incorporation of multivariate stochastic volatility in many empirical papers. In 2008 many economies went into recession and many of the associated policy discussions suggest that the parameters in VARs may be changing again.

Macroeconomic data sets typically involve monthly, quarterly or annual observations and, thus are only of moderate size. But VARs have a great number of parameters to estimate. This is particularly true if the number of dependent variables is more than two or three (as is required for an appropriate modelling of many macroeconomic relationships). Allowing for time-variation in VAR coefficients causes the number of parameters to proliferate. Allowing for the error covariance matrix to change over time only increases worries about over-parameterization. The research challenge facing macroeconomists is how to build models that are flexible enough to be empirically relevant, capturing key data features such as the Great Moderation, but not so flexible as to be seriously over-parameterized. Many approaches have been suggested, but a common theme in most of these is shrinkage. Whether for forecasting or estimation, it has been found that shrinkage can be of great benefit in reducing over-parameterization problems. This shrinkage can take the form of imposing restrictions on parameters or shrinking them towards zero. This has initiated a large increase in the use of Bayesian methods since prior information provides a logical and formally consistent way of introducing shrinkage. ${ }^{1}$ Furthermore, the computational tools necessary to carry out Bayesian estimation of high dimensional multivariate time series models have become well-developed and, thus, models which may have been difficult or impossible to estimate ten or twenty years ago can now be routinely used by macroeconomic practitioners.

A related class of models, and associated worries about over-parameterization, has arisen due to the increase in data availability. Macroeconomists are able to work with hundreds of different time series variables collected by government statistical agencies and other policy institutes. Building a model with hundreds of time series variables (with at most a few hundred observations on each) is a daunting task, raising the issue of a potential proliferation of parameters and a need for shrinkage or other methods for reducing the dimensionality of the model. Factor methods, where the information in the hundreds of variables is distilled into a few factors, are a popular way of dealing with this problem. Combining factor methods with VARs results in Factor-augmented VARs

\footnotetext{
${ }^{1}$ Prior information can be purely subjective. However, as will be discussed below, often empirical Bayesian or hierarchical priors are used by macroeconomists. For instance, the state equation in a state space model can be interpreted as a hierarchical prior. But, when we have limited data information relative to the number of parameters, the role of the prior becomes increasingly influential. In such cases, great care must to taken with prior elicitation.
} 
or FAVARs. However, just as with VARs, there is a need to allow for timevariation in parameters, which leads to an interest in TVP-FAVARs. Here, too, Bayesian methods are popular and for the same reason as with TVP-VARs: Bayesian priors provide a sensible way of avoiding over-parameterization problems and Bayesian computational tools are well-designed for dealing with such models.

In this monograph, we survey, discuss and extend the Bayesian literature on VARs, TVP-VARs and TVP-FAVARs with a focus on the practitioner. That is, we go beyond simply defining each model, but specify how to use them in practice, discuss the advantages and disadvantages of each and offer some tips on when and why each model can be used. In addition to this, we discuss some new modelling approaches for TVP-VARs. A website contains Matlab code which allows for Bayesian estimation of the models discussed in this monograph. Bayesian inference often involves the use of Markov chain Monte Carlo (MCMC) posterior simulation methods such as the Gibbs sampler. For many of the models, we provide complete details in this monograph. However, in some cases we only provide an outline of the MCMC algorithm. Complete details of all algorithms are given in a manual on the website.

Empirical macroeconomics is a very wide field and VARs, TVP-VARs and factor models, although important, are only some of the tools used in the field. It is worthwhile briefly mentioning what we are not covering in this monograph. There is virtually nothing in this monograph about macroeconomic theory and how it might infuse econometric modelling. For instance, Bayesian estimation of dynamic stochastic general equilibrium (DSGE) models is very popular. There will be no discussion of DSGE models in this monograph (see An and Schorfheide, 2007 or Del Negro and Schorfheide, 2010 for excellent treatments of Bayesian DSGE methods with Chib and Ramamurthy, 2010 providing a recent important advance in computation). Also, macroeconomic theory is often used to provide identifying restrictions to turn reduced form VARs into structural VARs suitable for policy analysis. We will not discuss structural VARs, although some of our empirical examples will provide impulse responses from structural VARs using standard identifying assumptions.

There is also a large literature on what might, in general, be called regimeswitching models. Examples include Markov switching VARs, threshold VARs, smooth transition VARs, floor and ceiling VARs, etc. These, although important, are not discussed here.

The remainder of this monograph is organized as follows. Section 2 provides discussion of VARs to develop some basic insights into the sorts of shrinkage priors (e.g. the Minnesota prior) and methods of finding empirically-sensible restrictions (e.g. stochastic search variable selection, or SSVS) that are used in empirical macroeconomics. Our goal is to extend these basic methods and priors used with VARs, to TVP variants. However, before considering these extensions, Section 3 discusses Bayesian inference in state space models using MCMC methods. We do this since TVP-VARs (including variants with multivariate stochastic volatility) are state space models and it is important that the practitioner knows the Bayesian tools associated with state space models before 
proceeding to TVP-VARs. Section 4 discusses Bayesian inference in TVP-VARs, including variants which combine the Minnesota prior or SSVS with the standard TVP-VAR. Section 5 discusses factor methods, beginning with the dynamic factor model, before proceeding to the factor augmented VAR (FAVAR) and TVP-FAVAR. Empirical illustrations are used throughout and Matlab code for implementing these illustrations (or, more generally, doing Bayesian inference in VARs, TVP-VARs and TVP-FAVARs) is available on the website associated with this monograph. ${ }^{2}$

\section{Bayesian VARs}

\subsection{Introduction and Notation}

The $\operatorname{VAR}(\mathrm{p})$ model can be written as:

$$
y_{t}=a_{0}+\sum_{j=1}^{p} A_{j} y_{t-j}+\varepsilon_{t}
$$

where $y_{t}$ for $t=1, . ., T$ is an $M \times 1$ vector containing observations on $M$ time series variables, $\varepsilon_{t}$ is an $M \times 1$ vector of errors, $a_{0}$ is an $M \times 1$ vector of intercepts and $A_{j}$ is an $M \times M$ matrix of coefficients. We assume $\varepsilon_{t}$ to be i.i.d. $N(0, \Sigma)$. Exogenous variables or more deterministic terms (e.g. deterministic trends or seasonals) can easily be added to the VAR and included in all the derivations below, but we do not do so to keep the notation as simple as possible.

The VAR can be written in matrix form in different ways and, depending on how this is done, some of the literature expresses results in terms of the multivariate Normal and others in terms of the matric-variate Normal distribution (see, e.g. Canova, 2007, and Kadiyala and Karlsson, 1997). The former arises if we use an $M T \times 1$ vector $y$ which stacks all $T$ observations on the first dependent variable, then all $T$ observations on the second dependent variable, etc.. The latter arises if we define $Y$ to be a $T \times M$ matrix which stacks the $T$ observations on each dependent variable in columns next to one another. $\varepsilon$ and $E$ denote stackings of the errors in a manner conformable to $y$ and $Y$, respectively. Define $x_{t}=\left(1, y_{t-1}^{\prime}, . ., y_{t-p}^{\prime}\right)$ and

$$
X=\left[\begin{array}{l}
x_{1} \\
x_{2} \\
\vdots \\
x_{T}
\end{array}\right]
$$

Note that, if we let $K=1+M p$ be the number of coefficients in each equation of the VAR, then $X$ is a $T \times K$ matrix.

Finally, if $A=\left(a_{0} A_{1} . . A_{p}\right)^{\prime}$ we define $\alpha=\operatorname{vec}(A)$ which is a $K M \times 1$ vector which stacks all the VAR coefficients (and the intercepts) into a vector. With all these definitions, we can write the VAR either as:

\footnotetext{
${ }^{2}$ The website address is: http://personal.strath.ac.uk/gary.koop/bayes_matlab_code_by_koop_and_korobilis.html
} 


$$
Y=X A+E
$$

or

$$
y=\left(I_{M} \otimes X\right) \alpha+\varepsilon,
$$

where $\varepsilon \sim N\left(0, \Sigma \otimes I_{T}\right)$.

The likelihood function can be derived from the sampling density, $p(y \mid \alpha, \Sigma)$. If it is viewed as a function of the parameters, then it can be shown to be of a form that breaks into two parts: one a distribution for $\alpha$ given $\Sigma$ and another where $\Sigma^{-1}$ has a Wishart distribution. ${ }^{3}$ That is,

$$
\alpha \mid \Sigma, y \sim N\left(\widehat{\alpha}, \Sigma \otimes\left(X^{\prime} X\right)^{-1}\right)
$$

and

$$
\Sigma^{-1} \mid y \sim W\left(S^{-1}, T-K-M-1\right),
$$

where $\widehat{A}=\left(X^{\prime} X\right)^{-1} X^{\prime} Y$ is the OLS estimate of $A$ and $\widehat{\alpha}=\operatorname{vec}(\widehat{A})$ and

$$
S=(Y-X \widehat{A})^{\prime}(Y-X \widehat{A})
$$

\subsection{Priors}

A variety of priors can be used with the VAR, of which we discuss some useful ones below. They differ in relation to three issues.

First, VARs are not parsimonious models. They have a great many coefficients. For instance, $\alpha$ contains $K M$ parameters which, for a $\operatorname{VAR}(4)$ involving 5 dependent variables is 105 . With quarterly macroeconomic data, the number of observations on each variable might be at most a few hundred. Without prior information, it is hard to obtain precise estimates of so many coefficients and, thus, features such as impulse responses and forecasts will tend to be imprecisely estimated (i.e. posterior or predictive standard deviations can be large). For this reason, it can be desirable to "shrink" forecasts and prior information offers a sensible way of doing this shrinkage. The priors discussed below differ in the way they achieve this goal.

Second, the priors used with VARs differ in whether they lead to analytical results for the posterior and predictive densities or whether MCMC methods are required to carry out Bayesian inference. With the VAR, natural conjugate priors lead to analytical results, which can greatly reduce the computational burden. Particularly if one is carrying out a recursive forecasting exercise which requires repeated calculation of posterior and predictive distributions, non-conjugate priors which require MCMC methods can be very computationally demanding.

\footnotetext{
${ }^{3}$ In this monograph, we use standard notational conventions to define all distributions such as the Wishart. See, among many other places, the appendix to Koop, Poirier and Tobias (2007). Wikipedia is also a quick and easy source of information about distributions.
} 
Third, the priors differ in how easily they can handle departures from the unrestricted VAR given in (1) such as allowing for different equations to have different explanatory variables, allowing for VAR coefficients to change over time, allowing for heteroskedastic structures for the errors of various sorts, etc. Natural conjugate priors typically do not lend themselves to such extensions.

\subsubsection{The Minnesota Prior}

Early work with Bayesian VARs with shrinkage priors was done by researchers at the University of Minnesota or the Federal Reserve Bank of Minneapolis (see Doan, Litterman and Sims, 1984 and Litterman, 1986). The priors they used have come to be known as Minnesota priors. They are based on an approximation which leads to great simplifications in prior elicitation and computation. This approximation involves replacing $\Sigma$ with an estimate, $\widehat{\Sigma}$. The original Minnesota prior simplifies even further by assuming $\Sigma$ to be a diagonal matrix. In this case, each equation of the VAR can be estimated one at a time and we can set $\widehat{\sigma}_{i i}=s_{i}^{2}$ (where $s_{i}^{2}$ is the standard OLS estimate of the error variance in the $i^{t h}$ equation and $\widehat{\sigma}_{i i}$ is the $i i^{t h}$ element of $\widehat{\Sigma}$ ). When $\Sigma$ is not assumed to be diagonal, a simple estimate such as $\widehat{\Sigma}=\frac{S}{T}$ can be used. A disadvantage of this approach is that it involves replacing an unknown matrix of parameters by an estimate (and potentially a poor one) rather than integrating it out in a Bayesian fashion. The latter strategy will lead to predictive densities which more accurately reflect parameter uncertainty. However, as we shall see below, replacing $\Sigma$ by an estimate simplifies computation since analytical posterior and predictive results are available. And it allows for a great range of flexibility in the choice of prior. If $\Sigma$ is not replaced by an estimate, the only fully Bayesian approach which leads to analytical results involves the use of a natural conjugate prior. As we shall see, the natural conjugate prior has some restrictive properties that may be unattractive in some cases.

When $\Sigma$ is replaced by an estimate, we only have to worry about a prior for $\alpha$ and the Minnesota prior assumes:

$$
\alpha \sim N\left(\underline{\alpha}_{M n}, \underline{V}_{M n}\right) .
$$

The Minnesota prior can be thought of as a way of automatically choosing $\underline{\alpha}_{M n}$ and $\underline{V}_{M n}$ in a manner which is sensible in many empirical contexts. To explain the Minnesota prior, note first that the explanatory variables in the VAR in any equation can be divided into the own lags of the dependent variable, the lags of the other dependent variables and exogenous or deterministic variables (in equation 1 the intercept is the only exogenous or deterministic variable, but in general there can be more such variables).

For the prior mean, $\underline{\alpha}_{M n}$, the Minnesota prior involves setting most or all of its elements to zero (thus ensuring shrinkage of the VAR coefficients towards zero and lessening the risk of over-fitting). When using growth rates data (e.g. GDP growth, the growth in the money supply, etc., which are typically found to be stationary and exhibit little persistence), it is sensible to simply set $\underline{\alpha}_{M n}=$ 
$0_{K M}$. However, when using levels data (e.g. GDP, the money supply, etc.) the Minnesota prior uses a prior mean expressing a belief that the individual variables exhibit random walk behavior. Thus, $\underline{\alpha}_{M n}=0_{K M}$ except for the elements corresponding to the first own lag of the dependent variable in each equation. These elements are set to one. These are the traditional choices for $\underline{\alpha}_{M n}$, but anything is possible. For instance, in our empirical illustration we set the prior mean for the coefficient on the first own lag to be 0.9 , reflecting a prior belief that our variables exhibit a fair degree of persistence, but not unit root behavior.

The Minnesota prior assumes the prior covariance matrix, $\underline{V}_{M n}$, to be diagonal. If we let $\underline{V}_{i}$ denote the block of $\underline{V}_{M n}$ associated with the $K$ coefficients in equation $i$ and $\underline{V}_{i, j j}$ be its diagonal elements, then a common implementation of the Minnesota prior would set:

$$
\underline{V}_{i, j j}=\left\{\begin{array}{l}
\frac{\underline{a}_{1}}{\underline{r}_{2}^{2}} \text { for coefficients on own lag } r \text { for } r=1, . ., p \\
\underline{\underline{a}}_{2} \sigma_{i i} \text { for coefficients on lag } r \text { of variable } j \neq i \text { for } r=1, . ., p . \\
\underline{a}_{3}^{2} \sigma_{i i} \text { for coefficients on exogenous variables }
\end{array}\right.
$$

This prior simplifies the complicated choice of fully specifying all the elements of $\underline{V}_{M n}$ to choosing three scalars, $\underline{a}_{1}, \underline{a}_{2}, \underline{a}_{3}$. This form captures the sensible properties that, as lag length increases, coefficients are increasingly shrunk towards zero and that (by setting $\underline{a}_{1}>\underline{a}_{2}$ ) own lags are more likely to be important predictors than lags of other variables. The exact choice of values for $\underline{a}_{1}, \underline{a}_{2}, \underline{a}_{3}$ depends on the empirical application at hand and the researcher may wish to experiment with different values for them. Typically, the researcher sets $\sigma_{i i}=s_{i}^{2}$. Litterman (1986) provides much additional motivation and discussion of these choices (e.g. an explanation for how the term $\frac{\sigma_{i i}}{\sigma_{j j}}$ adjusts for differences in the units that the variables are measured in).

Many variants of the Minnesota prior have been used in practice (e.g. Kadiyala and Karlsson, 1997, divide prior variances by $r$ instead of the $r^{2}$ which is used in (8)) as researchers make slight adjustments to tailor the prior for their particular application. The Minnesota prior has enjoyed a recent boom in popularity because of its simplicity and success in many applications, particularly involving forecasting. For instance, Banbura, Giannone and Reichlin (2010) use a slight modification of the Minnesota prior in a large VAR with over 100 dependent variables. Typically, factor methods are used with such large panels of data, but Banbura, Giannone and Reichlin (2010) find that the Minnesota prior leads to even better forecasting performance than factor methods.

A big advantage of the Minnesota prior is that it leads to simple posterior inference involving only the Normal distribution. It can be show that the posterior for $\alpha$ has the form:

$$
\alpha \mid y \sim N\left(\bar{\alpha}_{M n}, \bar{V}_{M n}\right)
$$

where 


$$
\bar{V}_{M n}=\left[\underline{V}_{M n}^{-1}+\left(\widehat{\Sigma}^{-1} \otimes\left(X^{\prime} X\right)\right)\right]^{-1}
$$

and

$$
\bar{\alpha}_{M n}=\bar{V}_{M n}\left[\underline{V}_{M n}^{-1} \underline{\alpha}_{M n}+\left(\widehat{\Sigma}^{-1} \otimes X\right)^{\prime} y\right] .
$$

But, as stressed above, a disadvantage of the Minnesota prior is that it does not provide a full Bayesian treatment of $\Sigma$ as an unknown parameter. Instead it simply plugs in $\Sigma=\widehat{\Sigma}$, ignoring any uncertainty in this parameter. In the remainder of this section we will discuss methods which treat $\Sigma$ as an unknown parameter. However, as we shall see, this means (apart from one restrictive special case) that analytical methods are not available and MCMC methods are required).

\subsubsection{Natural conjugate priors}

Natural conjugate priors are those where the prior, likelihood and posterior come from the same family of distributions. Our previous discussion of the likelihood function (see equations 5 and 6 ) suggests that, for the VAR, the natural conjugate prior has the form:

$$
\alpha \mid \Sigma \sim N(\underline{\alpha}, \Sigma \otimes \underline{V})
$$

and

$$
\Sigma^{-1} \sim W\left(\underline{S}^{-1}, \underline{\nu}\right)
$$

where $\underline{\alpha}, \underline{V}, \underline{\nu}$ and $\underline{S}$ are prior hyperparameters chosen by the researcher.

With this prior the posterior becomes:

$$
\alpha \mid \Sigma, y \sim N(\bar{\alpha}, \Sigma \otimes \bar{V})
$$

and

$$
\Sigma^{-1} \mid y \sim W\left(\bar{S}^{-1}, \bar{\nu}\right)
$$

where

$$
\begin{gathered}
\bar{V}=\left[\underline{V}^{-1}+X^{\prime} X\right]^{-1}, \\
\bar{A}=\bar{V}\left[\underline{V}^{-1} \underline{A}+X^{\prime} X \widehat{A}\right],
\end{gathered}
$$

$\bar{\alpha}=\operatorname{vec}(\bar{A})$

$$
\bar{S}=S+\underline{S}+\widehat{A}^{\prime} X^{\prime} X \widehat{A}+\underline{A}^{\prime} \underline{V}^{-1} \underline{A}-\bar{A}^{\prime}\left(\underline{V}^{-1}+X^{\prime} X\right) \bar{A}
$$

and

$$
\bar{\nu}=T+\underline{\nu} .
$$


In the previous formulae, we use notation where $\underline{A}$ is a $K \times M$ matrix made by unstacking the $K M \times 1$ vector $\underline{\alpha}$.

Posterior inference about the VAR coefficients can be carried out using the fact that the marginal posterior (i.e. after integrating out $\Sigma$ ) for $\alpha$ is a multivariate t-distribution. The mean of this t-distribution is $\bar{\alpha}$, its degrees of freedom parameter is $\bar{\nu}$ and its covariance matrix is:

$$
\operatorname{var}(\alpha \mid y)=\frac{1}{\bar{\nu}-M-1} \bar{S} \otimes \bar{V}
$$

These facts can be used to carry out posterior inference in this model.

The predictive distribution for $y_{T+1}$ in this model has an analytical form and, in particular, is multivariate-t with $\bar{\nu}$ degrees of freedom. The predictive mean of $y_{T+1}$ is $\left(x_{T+1} \bar{A}\right)^{\prime}$ which can be used to produce point forecasts. The predictive covariance matrix is $\frac{1}{\bar{\nu}-2}\left[1+x_{T+1} \bar{V} x_{T+1}^{\prime}\right] \bar{S}$. When forecasting more than one period ahead, an analytical formula for the predictive density does not exist. This means that either the direct forecasting method must be used (which turns the problem into one which only involves one step ahead forecasting) or predictive simulation is required.

Any values for the prior hyperparameters, $\underline{\alpha}, \underline{V}, \underline{\nu}$ and $\underline{S}$, can be chosen. The noninformative prior is obtained by setting $\underline{\nu}=\underline{S}=\underline{V}^{-1}=c I$ and letting $c \rightarrow 0$. It can be seen that this leads to posterior and predictive results which are based on familiar OLS quantities. The drawback of the noninformative prior is that it does not do any of the shrinkage which we have argued is so important for VAR modelling.

Thus, for the natural conjugate prior, analytical results exist which allow for Bayesian estimation and prediction. There is no need to use posterior simulation algorithms unless interest centers on nonlinear functions of the parameters (e.g. impulse response analysis such as those which arise in structural VARs, see Koop, 1992). The posterior distribution of, e.g., impulse responses can be obtained by Monte Carlo integration. That is, draws of $\Sigma^{-1}$ can be obtained from (13) and, conditional on these, draws of $\alpha$ can be taken from (12). ${ }^{4}$ Then draws of impulse responses can be calculated using these drawn values of $\Sigma^{-1}$ and $\alpha$.

However, there are two properties of this prior that can be undesirable in some circumstances. The first is that the $\left(I_{M} \otimes X\right)$ form of the explanatory variables in (4) means that every equation must have the same set of explanatory variables. For an unrestricted VAR this is fine, but is not appropriate if the researcher wishes to impose restrictions. Suppose, for instance, the researcher is working with a VAR involving variables such as output growth and the growth in the money supply and wants to impose a strict form of the neutrality of money. This would imply that the coefficients on the lagged money growth variables in the output growth equation are zero (but coefficients of lagged money growth in

\footnotetext{
${ }^{4}$ Alternatively, draws of $\alpha$ can be directly taken from its multivariate-t marginal posterior distribution.
} 
other equations would not be zero). Such restrictions cannot be imposed with the natural conjugate prior described here.

To explain the second possibly undesirable property of this prior, we introduce notation where individual elements of $\Sigma$ are denoted by $\sigma_{i j}$. The fact that the prior covariance matrix has the form $\Sigma \otimes \underline{V}$ (which is necessary to ensure natural conjugacy of the prior), implies that the prior covariance of the coefficients in equation $i$ is $\sigma_{i i} \underline{V}$. This means that the prior covariance of the coefficients in any two equations must be proportional to one another, a possibly restrictive feature. In our example, the researcher believing in the neutrality of money may wish to proceed as follows: in the output growth equation, the prior mean of the coefficients on lagged money growth variables should be zero and the prior covariance matrix should be very small (i.e. expressing a prior belief that these coefficients are very close to zero). In other equations, the prior covariance matrix on the coefficients on lagged money growth should be much larger. The natural conjugate prior does not allow us to use prior information of this form. It also does not allow us to use the Minnesota prior. That is, the Minnesota prior covariance matrix in (8) is written in terms of blocks which were labelled $\underline{V}_{i, j j}$ involving $i$ subscripts. That is, these blocks vary across equations which is not allowed for in the natural conjugate prior.

These two properties should be kept in mind when using the natural conjugate prior. There are generalizations of this natural conjugate prior, such as the extended natural conjugate prior of Kadiyala and Karlsson (1997), which surmount these problems. However, these lose the huge advantage of the natural conjugate prior described in this section: that analytical results are available and so no posterior simulation is required.

A property of natural conjugate priors is that, since the prior and likelihood have the same distributional form, the prior can be considered as arising from a fictitious sample. For instance, a comparison of (5) and (10) shows that $\widehat{\alpha}$ and $\left(X^{\prime} X\right)^{-1}$ in the likelihood play the same role as $\underline{\alpha}$ and $\underline{V}$ in the prior. The latter can be interpreted as arising from a fictitious sample (also called "dummy observations"), $Y_{0}$ and $X_{0}$ (e.g. $\underline{V}=\left(X_{0}^{\prime} X_{0}\right)^{-1}$ and $\underline{\alpha}$ based on an OLS estimate $\left.\left(X_{0}^{\prime} X_{0}\right)^{-1} X_{0}^{\prime} Y_{0}\right)$. This interpretation is developed in papers such as Sims (1993) and Sims and Zha (1998). On one level, this insight can simply serve as another way of motivating choices for $\underline{\alpha}$ and $\underline{V}$ as arising from particular choices for $Y_{0}$ and $X_{0}$. But papers such as Sims and Zha (1998) show how the dummy observation approach can be used to elicit priors for structural VARs. In this monograph, we will focus on the econometric as opposed to the macroeconomic issues. Accordingly, we will work with reduced form VARs and not say much about structural VARs. Here we only note that posterior inference in structural VARs is usually based on a reduced form VAR such as that discussed here, but then coefficients are transformed so as to give them a structural interpretation (see, e.g., Koop, 1992, for a simple example). For instance, structural VARs are often written as: 


$$
C_{0} y_{t}=c_{0}+\sum_{j=1}^{p} C_{j} y_{t-j}+u_{t}
$$

where $u_{t}$ is i.i.d. $N(0, I)$. Often appropriate identifying restrictions will provide a one-to-one mapping from the parameters of the reduced form VAR in (1) to the structural VAR. In this case, Bayesian inference can be done by using posterior simulation methods in the reduced form VAR and transforming each draw into a draw from the structural VAR. In models where such a one-to-one mapping does not exist (e.g. over-identified structural VARs) alternative methods of posterior inference exist (see Rubio-Ramirez, Waggoner and Zha, 2010).

While discussing such macroeconomic issues, it is worth noting that there is a growing literature that uses the insights of economic theory (e.g. from real business cycle or DSGE models) to elicit priors for VARs. Prominent examples include Ingram and Whiteman (1994) and Del Negro and Schorfheide (2004). We will not discuss this work in this monograph.

Finally, it is also worth mentioning the work of Villani (2009) on steady state priors for VARs. We have motivated prior information as being important as a way of ensuring shrinkage in an over-parameterized VAR. However, most of the shrinkage discussed previously relates to the VAR coefficients. Often researchers have strong prior information about the unconditional means (i.e. the steady states) of the variables in their VARs. It is desirable to include such information as an additional source of shrinkage in the VAR. However, it is not easy to do this in the VAR in (1) since the intercepts cannot be directly interpreted as the unconditional means of the variables in the VAR. Villani (2009) recommends writing the VAR as:

$$
\widetilde{A}(L)\left(y_{t}-\widetilde{a}_{0}\right)=\varepsilon_{t}
$$

where $\widetilde{A}(L)=I-\widetilde{A}_{1} L-. .-\widetilde{A}_{p} L^{p}, L$ is the lag operator and $\varepsilon_{t}$ is i.i.d. $N(0, \Sigma)$. In this parameterization, $\widetilde{a}_{0}$ can be interpreted as the vector of unconditional means of the dependent variables and a prior placed on it reflecting the researcher's beliefs about steady state values for them. For $\widetilde{A}(L)$ and $\Sigma$ one of the priors described previously (or below) can be used. A drawback of this approach is that an analytical form for the posterior no longer exists. However, Villani (2009) develops a Gibbs sampling algorithm for carrying out Bayesian inference in this model.

\subsubsection{The Independent Normal-Wishart Prior}

The natural conjugate prior has the large advantage that analytical results are available for posterior inference and prediction. However, it does have the drawbacks noted previously (i.e. it assumes each equation to have the same explanatory variables and it restricts the prior covariance of the coefficients in any two equations to be proportional to one another). Accordingly, in this section, we introduce a more general framework for VAR modelling. Bayesian inference in these models will require posterior simulation algorithms such as the Gibbs sampler. The natural conjugate prior had $\alpha \mid \Sigma$ being Normal and $\Sigma^{-1}$ being 
Wishart. Note that the fact that the prior for $\alpha$ depends on $\Sigma$ implies that $\alpha$ and $\Sigma$ are not independent of one another. In this section, we work with a prior which has VAR coefficients and the error covariance being independent of one another (hence the name "independent Normal-Wishart prior").

To allow for different equations in the VAR to have different explanatory variables, we have to modify our previous notation slightly. To avoid any possibility of confusion, we will use " $\beta$ " as notation for VAR coefficients in this restricted VAR model instead of $\alpha$. We write each equation of the VAR as:

$$
y_{m t}=z_{m t}^{\prime} \beta_{m}+\varepsilon_{m t},
$$

with $t=1, . ., T$ observations for $m=1, . ., M$ variables. $y_{m t}$ is the $t^{t h}$ observation on the $m^{t h}$ variable, $z_{m t}$ is a $k_{m}$-vector containing the $t^{t h}$ observation of the vector of explanatory variables relevant for the $m^{t h}$ variable and $\beta_{m}$ is the accompanying $k_{m}$-vector of regression coefficients. Note that if we had $z_{m t}=$ $\left(1, y_{t-1}^{\prime}, . ., y_{t-p}^{\prime}\right)^{\prime}$ for $m=1, . ., M$ then we would obtain the unrestricted VAR of the previous section. However, by allowing for $z_{m t}$ to vary across equations we are allowing for the possibility of a restricted VAR (i.e. it allows for some of the coefficients on the lagged dependent variables to be restricted to zero).

We can stack all equations into vectors/matrices as $y_{t}=\left(y_{1 t}, . ., y_{M t}\right)^{\prime}, \varepsilon_{t}=$ $\left(\varepsilon_{1 t}, . ., \varepsilon_{M t}\right)^{\prime}$

$$
\begin{gathered}
\beta=\left(\begin{array}{c}
\beta_{1} \\
\vdots \\
\beta_{M}
\end{array}\right), \\
Z_{t}=\left(\begin{array}{cccc}
z_{1 t}^{\prime} & 0 & \cdots & 0 \\
0 & z_{2 t}^{\prime} & \ddots & \vdots \\
\vdots & \ddots & \ddots & 0 \\
0 & \cdots & 0 & z_{M t}^{\prime}
\end{array}\right),
\end{gathered}
$$

where $\beta$ is a $k \times 1$ vector and $Z_{t}$ is $M \times k$ where $k=\sum_{j=1}^{M} k_{j}$. As before, we assume $\varepsilon_{t}$ to be i.i.d. $N(0, \Sigma)$.

Using this notation, we can write the (possibly restricted) VAR as:

$$
y_{t}=Z_{t} \beta+\varepsilon_{t} .
$$

Stacking as:

$$
\begin{aligned}
& y=\left(\begin{array}{c}
y_{1} \\
\vdots \\
y_{T}
\end{array}\right), \\
& \varepsilon=\left(\begin{array}{c}
\varepsilon_{1} \\
\vdots \\
\varepsilon_{T}
\end{array}\right),
\end{aligned}
$$




$$
Z=\left(\begin{array}{c}
Z_{1} \\
\vdots \\
Z_{T}
\end{array}\right)
$$

we can write

$$
y=Z \beta+\varepsilon
$$

and $\varepsilon$ is $N(0, I \otimes \Sigma)$.

It can be seen that the restricted VAR can be written as a Normal linear regression model with an error covariance matrix of a particular form. A very general prior for this model (which does not involve the restrictions inherent in the natural conjugate prior) is the independent Normal-Wishart prior:

$$
p\left(\beta, \Sigma^{-1}\right)=p(\beta) p\left(\Sigma^{-1}\right)
$$

where

$$
\beta \sim N\left(\underline{\beta}, \underline{V}_{\beta}\right)
$$

and

$$
\Sigma^{-1} \sim W\left(\underline{S}^{-1}, \underline{\nu}\right) .
$$

Note that this prior allows for the prior covariance matrix, $\underline{V}_{\beta}$, to be anything the researcher chooses, rather than the restrictive $\Sigma \otimes \underline{V}$ form of the natural conjugate prior. For instance, the researcher could set $\beta$ and $\underline{V}_{\beta}$ exactly as in the Minnesota prior. A noninformative prior can be obtained by setting $\underline{\nu}=\underline{S}=\underline{V}_{\beta}^{-1}=0$.

Using this prior, the joint posterior $p\left(\beta, \Sigma^{-1} \mid y\right)$ does not have a convenient form that would allow easy Bayesian analysis (e.g. posterior means and variances do not have analytical forms). However, the conditional posterior distributions $p\left(\beta \mid y, \Sigma^{-1}\right)$ and $p\left(\Sigma^{-1} \mid y, \beta\right)$ do have convenient forms:

$$
\beta \mid y, \Sigma^{-1} \sim N\left(\bar{\beta}, \bar{V}_{\beta}\right),
$$

where

$$
\bar{V}_{\beta}=\left(\underline{V}_{\beta}^{-1}+\sum_{t=1}^{T} Z_{t}^{\prime} \Sigma^{-1} Z_{t}\right)^{-1}
$$

and

$$
\bar{\beta}=\bar{V}_{\beta}\left(\underline{V}_{\beta}^{-1} \underline{\beta}+\sum_{i=1}^{T} Z_{t}^{\prime} \Sigma^{-1} y_{t}\right) .
$$

Furthermore, 


$$
\Sigma^{-1} \mid y, \beta \sim W\left(\bar{S}^{-1}, \bar{\nu},\right)
$$

where

$$
\bar{\nu}=T+\underline{\nu}
$$

and

$$
\bar{S}=\underline{S}+\sum_{t=1}^{T}\left(y_{t}-Z_{t} \beta\right)\left(y_{t}-Z_{t} \beta\right)^{\prime} .
$$

Accordingly, a Gibbs sampler which sequentially draws from the Normal $p(\beta \mid y, \Sigma)$ and the Wishart $p\left(\Sigma^{-1} \mid y, \beta\right)$ can be programmed up in a straightforward fashion. As with any Gibbs sampler, the resulting posterior simulator output can be used to calculate posterior properties of any function of the parameters, marginal likelihoods (for model comparison) and/or to do prediction.

Note that, for the VAR, $Z_{\tau}$ will contain lags of variables and, thus, contain information dated $\tau-1$ or earlier. The one-step ahead predictive density (i.e. the one for predicting at time $\tau$ given information through $\tau-1$ ), conditional on the parameters of the model is:

$$
y_{\tau} \mid Z_{\tau}, \beta, \Sigma \sim N\left(Z_{t} \beta, \Sigma\right) .
$$

This result, along with a Gibbs sampler producing draws $\beta^{(r)}, \Sigma^{(r)}$ for $r=1, \ldots, R$ allows for predictive inference. ${ }^{5}$ For instance, the predictive mean (a popular point forecast) could be obtained as:

$$
E\left(y_{\tau} \mid Z_{\tau}\right)=\frac{\sum_{r=1}^{R} Z_{t} \beta^{(r)}}{R}
$$

and other predictive moments can be calculated in a similar fashion. Alternatively, predictive simulation can be done at each Gibbs sampler draw, but this can be computationally demanding. For forecast horizons greater than one, the direct method can be used. This strategy for doing predictive analysis can be used with any of the priors or models discussed below.

\subsubsection{Stochastic Search Variable Selection (SSVS) in VARs}

SSVS as Implemented in George, Sun and Ni (2008) In the previous sections, we have described various priors for unrestricted and restricted VARs which allow for shrinkage of VAR coefficients. However, these approaches required substantial prior input from the researcher (although this prior input can be of an automatic form such as in the Minnesota prior). There is another prior that, in a sense, does shrinkage and leads to restricted VARs, but does so in an automatic fashion that requires only minimal prior input from the researcher.

\footnotetext{
${ }^{5}$ Typically, some initial draws are discarded as the "burn in". Accordingly, $r=1, . ., R$ should be the post-burn in draws.
} 
The methods associated with this prior are called SSVS and are enjoying increasing popularity and, accordingly, we describe them here in detail. SSVS can be done in several ways. Here we describe the implementation of George, Sun and $\mathrm{Ni}(2008)$.

The basic idea underlying SSVS can be explained quite simply. Suppose $\alpha_{j}$ is a VAR coefficient. Instead of simply using a prior for it as before (e.g. as in (10), SSVS specifies a hierarchical prior (i.e. a prior expressed in terms of parameters which in turn have a prior of their own) which is a mixture of two Normal distributions:

$$
\alpha_{j} \mid \gamma_{j} \sim\left(1-\gamma_{j}\right) N\left(0, \kappa_{0 j}^{2}\right)+\gamma_{j} N\left(0, \kappa_{1 j}^{2}\right),
$$

where $\gamma_{j}$ is a dummy variable. If $\gamma_{j}$ equals one then $\alpha_{j}$ is drawn from the second Normal and if it equals zero then $\alpha_{j}$ is drawn from the first Normal. The prior is hierarchical since $\gamma_{j}$ is treated as an unknown parameter and estimated in a data-based fashion. The SSVS aspect of this prior arises by choosing the first prior variance, $\kappa_{0 j}^{2}$, to be "small" (so that the coefficient is constrained to be virtually zero) and the second prior variance, $\kappa_{1 j}^{2}$, to be "large" (implying a relatively noninformative prior for the corresponding coefficient). Below we describe what George, Sun and Ni (2008) call a "default semi-automatic approach" to choosing $\kappa_{0 j}^{2}$ and $\kappa_{1 j}^{2}$ which requires minimal subjective prior information from the researcher.

The SSVS approach can be thought of as automatically selecting a restricted VAR since it can, in a data-based fashion, set $\gamma_{j}=0$ and (to all intents and purposes) delete the corresponding lagged dependent variable form the model. Alternatively, SSVS can be thought of as a way of doing shrinkage since VAR coefficients can be shrunk to zero.

The researcher can also carry out a Bayesian unrestricted VAR analysis using an SSVS prior, then use the output from this analysis to select a restricted VAR (which can then be estimated using, e.g., a noninformative or an independent Normal-Wishart prior). This can be done by using the posterior $p(\gamma \mid Y)$ where $\gamma=\left(\gamma_{1}, . ., \gamma_{K M}\right)^{\prime}$. One common strategy is to use $\widehat{\gamma}$, the mode of $p(\gamma \mid Y)$. This will be a vector of zeros and ones and the researcher can simply omit explanatory variables corresponding to the zeros. The relationship between such a strategy and conventional model selection techniques using an information criteria (e.g. the Akaike or Bayesian information criteria) is discussed in Fernandez, Ley and Steel (2001). Alternatively, if the MCMC algorithm described below is simply run and posterior results for the VAR coefficients calculated using the resulting MCMC output, the result will be Bayesian model averaging (BMA).

In this section, we focus on SSVS, but it is worth mentioning that there are many other Bayesian methods for selecting a restricted model or doing BMA. In cases where the number of models under consideration is small, it is possible to simply calculate the marginal likelihoods for every model and use these as weights when doing BMA or simply to select the single model with the highest marginal likelihood. Marginal likelihoods for the multivariate time series models discussed in this monograph can be calculated in several ways (see Section 3). 
In cases (such as the present ones) where the number of restricted models is very large, various other approaches have been suggested, see Green (1995) and Carlin and Chib (1995). See also Chipman, George and McCulloch (2001) for a survey on Bayesian model selection and, in particular, the discussion of practical issues is prior elicitation and posterior simulation that arise.

SSVS allows us to work with the unrestricted VAR and have the algorithm pick out an appropriate restricted VAR. Accordingly we will return to our notation for the unrestricted VAR (see Section 2.1). The unrestricted VAR is written in (3) and $\alpha$ is the $K M \times 1$ vector of VAR coefficients. SSVS can be interpreted as defining a hierarchical prior for all of the elements of $\alpha$ and $\Sigma$. The prior for $\alpha$ given in (21) can be written more compactly as:

$$
\alpha \mid \gamma \sim N(0, D D)
$$

where $D$ is a diagonal matrix with $(j, j)^{t h}$ element given by $d_{j}$ where

$$
d_{j}=\left\{\begin{array}{l}
\kappa_{0 j} \text { if } \gamma_{j}=0 \\
\kappa_{1 j} \text { if } \gamma_{j}=1
\end{array}\right.
$$

Note that this prior implies a mixture of two Normals as written in (21).

George, Sun and Ni (2008) describe a "default semi-automatic approach" to selecting the prior hyperparameters $\kappa_{0 j}$ and $\kappa_{1 j}$ which involves setting $\kappa_{0 j}=$ $c_{0} \sqrt{\widehat{\operatorname{var}}\left(\alpha_{j}\right)}$ and $\kappa_{1 j}=c_{1} \sqrt{\widehat{\operatorname{var}}\left(\alpha_{j}\right)}$ where $\widehat{\operatorname{var}}\left(\alpha_{j}\right)$ is an estimate of the variance of the coefficient in an unrestricted VAR (e.g. the ordinary least squares quantity or an estimate based on a preliminary Bayesian estimation the VAR using a noninformative prior). The pre-selected constants $c_{0}$ and $c_{1}$ must have $c_{0} \ll c_{1}$ (e.g. $c_{0}=0.1$ and $c_{1}=10$ ).

For $\gamma=\left(\gamma_{1}, . ., \gamma_{K M}\right)^{\prime}$, the SSVS prior assumes that each element has a Bernoulli form (independent of the other elements of $\gamma$ ) and, hence, for $j=$ $1, . ., K M$, we have

$$
\begin{aligned}
& \operatorname{Pr}\left(\gamma_{j}=1\right)=\underline{q}_{j} \\
& \operatorname{Pr}\left(\gamma_{j}=0\right)=1-\underline{q}_{j} .
\end{aligned}
$$

A natural default choice is $\underline{q}_{j}=0.5$ for all $j$, implying each coefficient is a priori equally likely to be included as excluded.

So far, we have said nothing about the prior for $\Sigma$ and (for the sake of brevity) we will not provide details relating to it. Suffice it to note here that if a Wishart prior for $\Sigma^{-1}$ like (18) is used, then a formula very similar to (20) can be used as a block in a Gibbs sampling algorithm. Alternatively, George, Sun and $\mathrm{Ni}$ (2008) use a prior for $\Sigma$ which allows for them to do SSVS on the error covariance matrix. That is, although they always assume the diagonal elements of $\Sigma$ are positive (so as to ensure a positive definite error covariance matrix), they allow for parameters which determine the off-diagonal elements to have an SSVS prior thus allowing for restrictions to be imposed on $\Sigma$. We refer the interested reader to George, Sun and Ni (2008) or the manual on the website associated with this monograph for details. 
Posterior computation in the VAR with SSVS prior can be carried out using a Gibbs sampling algorithm. For the VAR coefficients we have

$$
\alpha \mid y, \gamma, \Sigma \sim N\left(\bar{\alpha}_{\alpha}, \bar{V}_{\alpha}\right),
$$

where

$$
\begin{gathered}
\bar{V}_{\alpha}=\left[\Sigma^{-1} \otimes\left(X^{\prime} X\right)+(D D)^{-1}\right]^{-1}, \\
\bar{\alpha}_{\alpha}=\bar{V}_{\alpha}\left[\left(\Psi \Psi^{\prime}\right) \otimes\left(X^{\prime} X\right) \hat{\alpha}\right], \\
\hat{A}=\left(X^{\prime} X\right)^{-1} X^{\prime} Y
\end{gathered}
$$

and

$$
\hat{\alpha}=\operatorname{vec}(\hat{A}) .
$$

The conditional posterior for $\gamma$ has $\gamma_{j}$ being independent Bernoulli random variables:

$$
\begin{aligned}
& \operatorname{Pr}\left[\gamma_{j}=1 \mid y, \alpha\right]=\bar{q}_{j}, \\
& \operatorname{Pr}\left[\gamma_{j}=0 \mid y, \alpha\right]=1-\bar{q}_{j},
\end{aligned}
$$

where

$$
\bar{q}_{j}=\frac{\frac{1}{\kappa_{1 j}} \exp \left(-\frac{\alpha_{j}^{2}}{2 \kappa_{1 j}^{2}}\right) \underline{q}_{j}}{\frac{1}{\kappa_{1 j}} \exp \left(-\frac{\alpha_{j}^{2}}{2 \kappa_{1 j}^{2}}\right) \underline{q}_{j}+\frac{1}{\kappa_{0 j}} \exp \left(-\frac{\alpha_{j}^{2}}{2 \kappa_{0 j}^{2}}\right)\left(1-\underline{q}_{j}\right)} .
$$

Thus, a Gibbs sampler involving the Normal distribution and the Bernoulli distribution (and either the Gamma or Wishart distributions depending on what prior is used for $\Sigma^{-1}$ ) allows for posterior inference in this model.

SSVS as Implemented in Korobilis (2009b) The implementation of SSVS just described is a popular one. However, there are other similar methods for automatic model selection in VARs. In particular, the approach of George, Sun and Ni (2008) involves selecting values for the "small" prior variance $\kappa_{0 j}$. The reader may ask why not set "small" exactly equal to zero? This has been done in regression models in papers such as Kuo and Mallick (1997) through restricting coefficients to be precisely zero if $\gamma_{j}=0$. There are some subtle statistical issues which arise when doing this. ${ }^{6}$ Korobilis (2009b) has extended the use of such methods to VARs. Since, unlike the implementation of George, Sun and

\footnotetext{
${ }^{6}$ For instance, asympotically such priors will always set $\gamma_{j}=1$ for all $j$.
} 
Ni (2008), this approach leads to restricted VARs (as opposed to unrestricted VARs with very tight priors on some of the VAR coefficients), we return to our notation for restricted VARs and modify it slightly. In particular, replace (16) by

$$
y_{t}=Z_{t} \widetilde{\beta}+\varepsilon_{t} .
$$

where $\widetilde{\beta}=\widetilde{D} \beta$ and $\widetilde{D}$ is a diagonal matrix with the $j^{\text {th }}$ diagonal element being $\gamma_{j}$ (where, as before, $\gamma_{j}$ is a dummy variable). In words, this model allows for each VAR coefficient to be set to zero (if $\gamma_{j}=0$ ) or included in an unrestricted fashion (if $\gamma_{j}=1$ ).

Bayesian inference using the prior can be carried out in a straightforward fashion. For exact details on the necessary MCMC algorithm, see Korobilis (2009b) and the manual on the website associated with this book. However, the idea underlying this algorithm can be explained quite simply. Conditional on $\gamma$, this model is a restricted VAR and the MCMC algorithm of Section 2.2.2 for the independent Normal-Wishart prior can be used. Thus, all that is required is a method for taking draws from $\gamma$ (conditional on the parameters of the VAR). Korobilis (2009b) derives the necessary distribution.

\subsection{Empirical Illustration of Bayesian VAR Methods}

To illustrate Bayesian VAR methods using some of the priors and methods described above, we use a quarterly US data set on the inflation rate $\Delta \pi_{t}$ (the annual percentage change in a chain-weighted GDP price index), the unemployment rate $u_{t}$ (seasonally adjusted civilian unemployment rate, all workers over age 16) and the interest rate $r_{t}$ (yield on the three month Treasury bill rate). Thus $y_{t}=\left(\Delta \pi_{t}, u_{t}, r_{t}\right)^{\prime}$. The sample runs from 1953Q1 to 2006Q3. These three variables are commonly used in New Keynesian VARs. ${ }^{7}$ Examples of papers which use these, or similar, variables include Cogley and Sargent (2005), Primiceri (2005) and Koop, Leon-Gonzalez and Strachan (2009). The data are plotted in Figure 1.

\footnotetext{
${ }^{7}$ The data are obtained from the Federal Reserve Bank of St. Louis website, http://research.stlouisfed.org/fred2/.
} 


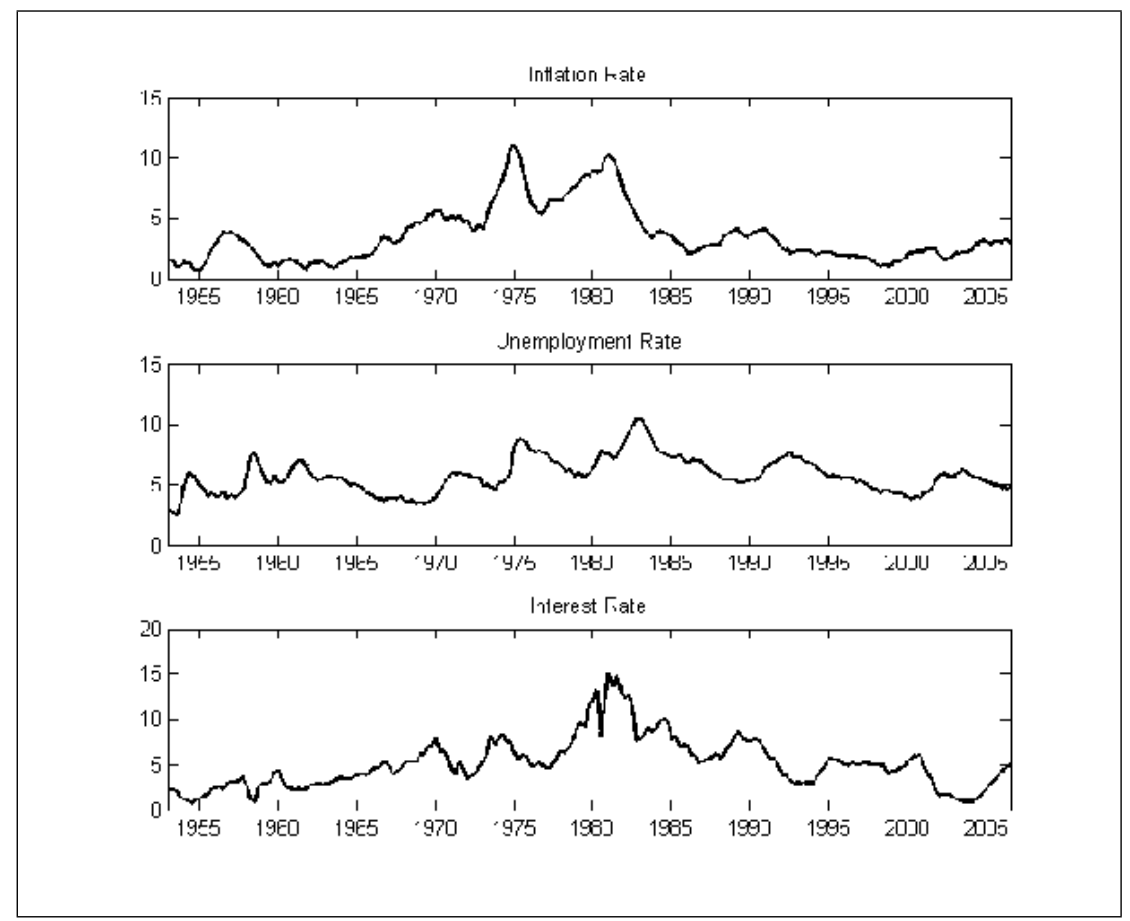

Figure 1: Data Used In Empirical Illustration

To illustrate Bayesian VAR analysis using this data, we work with an unrestricted VAR with an intercept and four lags of all variables included in every equation and consider the following six priors:

- Noninformative: Noninformative version of natural conjugate prior (equations 10 and 11 with $\underline{\alpha}=0_{K M \times 1}, \underline{V}=100 I_{K \times K}, \underline{v}=0$ and $\left.\underline{S}=0_{M \times M}\right)$.

- Natural conjugate: Informative natural conjugate prior with subjectively chosen prior hyperparameters (equations 10 and 11 with $\underline{\alpha}=0_{K M \times 1}$, $\underline{V}=10 I_{K}, \underline{v}=M+1$ and $\left.\underline{S}^{-1}=I_{M}\right)$.

- Minnesota: Minnesota prior (equations 7 and 8, where $\underline{\alpha}_{M n}$ is zero, except for the first own lag of each variable which is $0.9 . \Sigma$ is diagonal with elements $s_{i}^{2}$ obtained from univariate regressions of each dependent variable on an intercept and four lags of all variables).

- Independent Normal-Wishart: Independent Normal-Wishart prior with subjectively chosen prior hyperparameters (equations 17 and 18 with $\underline{\beta}=$ $0_{K M \times 1}, \underline{V}_{\beta}=10 I_{K M}, \underline{v}=M+1$ and $\left.\underline{S}^{-1}=I_{M}\right)$.

- SSVS-VAR: SSVS prior for VAR coefficients (with default semi-automatic approach prior with $c_{0}=0.1$ and $c_{1}=10$ ) and Wishart prior for $\Sigma^{-1}$ (equation 18 with $\underline{v}=M+1$ and $\underline{S}^{-1}=I_{M}$ ). 
- SSVS: SSVS on both VAR coefficients and error covariance (default semiautomatic approach). ${ }^{8}$

For the first three priors, analytical posterior and predictive results are available. For the last three, posterior and predictive simulation is required. The results below are based on 50000 MCMC draws, for which the first 20000 are discarded as burn-in draws. For impulse responses (which are nonlinear functions of the VAR coefficients and $\Sigma$ ), posterior simulation methods are required for all six priors.

With regards to impulse responses, they are identified by assuming $C_{0}$ in (14) is lower triangular and the dependent variables are ordered as: inflation, unemployment and interest rate. This is a standard identifying assumption used, among many others, by Bernanke and Mihov (1998), Christiano, Eichanbaum and Evans (1999) and Primiceri (2005). It allows for the interpretation of the interest rate shock as a monetary policy shock.

With VARs, the parameters themselves (as opposed to functions of them such as impulse responses) are rarely of direct interest. In addition, the fact that there are so many of them makes it hard for the reader to interpret tables of VAR coefficients. Nevertheless, Table 1 presents posterior means of all the VAR coefficients for two priors: the noninformative one and SSVS prior. Note that they are yielding similar results, although there is some evidence that SSVS is slightly shrinking the coefficients towards zero.

\begin{tabular}{|l|l|l|l|l|l|l|}
\hline \multicolumn{7}{|c|}{ Table 1. Posterior mean of VAR Coefficients for Two Priors } \\
\hline & Noninformative & \multicolumn{4}{l|}{ SSVS - VAR } \\
\hline & $\Delta \pi_{t}$ & $u_{t}$ & $r_{t}$ & $\Delta \pi_{t}$ & $u_{t}$ & $r_{t}$ \\
\hline Intercept & 0.2920 & 0.3222 & -0.0138 & 0.2053 & 0.3168 & 0.0143 \\
\hline$\Delta \pi_{t-1}$ & 1.5087 & 0.0040 & 0.5493 & 1.5041 & 0.0044 & 0.3950 \\
\hline$u_{t-1}$ & -0.2664 & 1.2727 & -0.7192 & -0.142 & 1.2564 & -0.5648 \\
\hline$r_{t-1}$ & -0.0570 & -0.0211 & 0.7746 & -0.0009 & -0.0092 & 0.7859 \\
\hline$\Delta \pi_{t-2}$ & -0.4678 & 0.1005 & -0.7745 & -0.5051 & 0.0064 & -0.226 \\
\hline$u_{t-2}$ & 0.1967 & -0.3102 & 0.7883 & 0.0739 & -0.3251 & 0.5368 \\
\hline$r_{t-2}$ & 0.0626 & -0.0229 & -0.0288 & 0.0017 & -0.0075 & -0.0004 \\
\hline$\Delta \pi_{t-3}$ & -0.0774 & -0.1879 & 0.8170 & -0.0074 & 0.0047 & 0.0017 \\
\hline$u_{t-3}$ & -0.0142 & -0.1293 & -0.3547 & 0.0229 & -0.0443 & -0.0076 \\
\hline$r_{t-3}$ & -0.0073 & 0.0967 & 0.0996 & -0.0002 & 0.0562 & 0.1119 \\
\hline$\Delta \pi_{t-4}$ & 0.0369 & 0.1150 & -0.4851 & -0.0005 & 0.0028 & -0.0575 \\
\hline$u_{t-4}$ & 0.0372 & 0.0669 & 0.3108 & 0.0160 & 0.0140 & 0.0563 \\
\hline$r_{t-4}$ & -0.0013 & -0.0254 & 0.0591 & -0.0011 & -0.0030 & 0.0007 \\
\hline
\end{tabular}

Remember that SSVS allows to the calculation of $\operatorname{Pr}\left(\gamma_{j}=1 \mid y\right)$ for each VAR coefficient and such posterior inclusion probabilities can be used either in model averaging or as an informal measure of whether an explanatory variable should be included or not. Table 2 presents such posterior inclusion probabilities

\footnotetext{
${ }^{8} \mathrm{SSVS}$ on the non-diagonal elements of $\Sigma$ is not fully described in this monograph. See George, Sun and Ni (2008) for complete details.
} 
using the SSVS-VAR prior. The empirical researcher may wish to present such a table for various reasons. For instance, if the researcher wishes to select a single restricted VAR which only includes coefficients with $\operatorname{Pr}\left(\gamma_{j}=1 \mid y\right)>\frac{1}{2}$, then he would work with a model which restricts 25 of 39 coefficients to zero. Table 2 shows which coefficients are important. Of the 14 included coefficients two are intercepts and three are first own lags in each equation. The researcher using SSVS to select a single model would restrict most of the remaining VAR coefficients to be zero. The researcher using SSVS to do model averaging would, in effect, be restricting them to be approximately zero. Note also that SSVS can be used to do lag length selection in an automatic fashion. None of the coefficients on the fourth lag variables is found to be important and only one of nine possible coefficients on third lags is found to be important.

\begin{tabular}{|l|l|l|l|}
\hline \multicolumn{4}{|c|}{ Table 2. Posterior Inclusion Probabilities for } \\
VAR Coefficients: SSVS-VAR Prior \\
\hline & $\Delta \pi_{t}$ & $u_{t}$ & $r_{t}$ \\
\hline Intercept & 0.7262 & 0.9674 & 0.1029 \\
\hline$\Delta \pi_{t-1}$ & 1 & 0.0651 & 0.9532 \\
\hline$u_{t-1}$ & 0.7928 & 1 & 0.8746 \\
\hline$r_{t-1}$ & 0.0612 & 0.2392 & 1 \\
\hline$\Delta \pi_{t-2}$ & 0.9936 & 0.0344 & 0.5129 \\
\hline$u_{t-2}$ & 0.4288 & 0.9049 & 0.7808 \\
\hline$r_{t-2}$ & 0.0580 & 0.2061 & 0.1038 \\
\hline$\Delta \pi_{t-3}$ & 0.0806 & 0.0296 & 0.1284 \\
\hline$u_{t-3}$ & 0.2230 & 0.2159 & 0.1024 \\
\hline$r_{t-3}$ & 0.0416 & 0.8586 & 0.6619 \\
\hline$\Delta \pi_{t-4}$ & 0.0645 & 0.0507 & 0.2783 \\
\hline$u_{t-4}$ & 0.2125 & 0.1412 & 0.2370 \\
\hline$r_{t-4}$ & 0.0556 & 0.1724 & 0.1097 \\
\hline
\end{tabular}

With VARs, the researcher is often interested in forecasting. It is worth mentioning that often recursive forecasting exercises, which involve forecasting at time $\tau=\tau_{0}, . ., T$, are often done. These typically involve estimating a model $T-\tau_{0}$ times using appropriate sub-samples of the data. If MCMC methods are required, this can be computationally demanding. That is, running an MCMC algorithm $T-\tau_{0}$ times can (depending on the model and application) be very slow. If this is the case, then the researcher may be tempted to work with methods which do not require MCMC such as the Minnesota or natural conjugate priors. Alternatively, sequential importance sampling methods such as the particle filter (see, e.g. Doucet, Godsill and Andrieu, 2000 or Johannes and Polson, 2009) can be used which do not require the MCMC algorithm to be run at each point in time. ${ }^{9}$

Table 3 presents predictive results for an out-of-sample forecasting exercise based on the predictive density $p\left(y_{T+1} \mid y_{1} . ., y_{T}\right)$ where $T=2006 Q 3$. It can be

\footnotetext{
${ }^{9}$ Although the use of particle filtering raises empirical challenges of its own which we will not discuss here.
} 
seen that for this empirical example, which involves a moderately large data set, the prior is having relatively little impact. That is, predictive means and standard deviations are similar for all six priors, although it can be seen that the predictive standard deviations with the Minnesota prior do tend to be slightly smaller than the other priors.

\begin{tabular}{|l|c|c|c|}
\hline \multicolumn{4}{|c|}{ Table 3. Predictive mean of $y_{T+1}$ (st. dev. in parentheses) } \\
\hline PRIOR & $\Delta \pi_{T+1}$ & $u_{T+1}$ & $r_{T+1}$ \\
\hline \multirow{2}{*}{ Noninformative } & 3.105 & 4.610 & 4.382 \\
& $(0.315)$ & $(0.318)$ & $(0.776)$ \\
\hline \multirow{2}{*}{ Minnesota } & 3.124 & 4.628 & 4.350 \\
& $(0.302)$ & $(0.319)$ & $(0.741)$ \\
\hline \multirow{2}{*}{ Natural conjugate } & 3.106 & 4.611 & 4.380 \\
& $(0.313)$ & $(0.314)$ & $(0.748)$ \\
\hline \multirow{2}{*}{ Indep. Normal-Wishart } & 3.110 & 4.622 & 4.315 \\
& $(0.322)$ & $(0.324)$ & $(0.780)$ \\
\hline \multirow{2}{*}{ SSVS - VAR } & 3.097 & 4.641 & 4.281 \\
& $(0.323)$ & $(0.323)$ & $(0.787)$ \\
\hline \multirow{2}{*}{ SSVS } & 3.108 & 4.639 & 4.278 \\
& $(0.304)$ & $(0.317)$ & $(0.785)$ \\
\hline True value, $y_{T+1}$ & 3.275 & 4.700 & 4.600 \\
\hline
\end{tabular}

Figures 2 and 3 present impulse responses of all three of our variables to all three of the shocks for two of the priors: the noninformative one and the SSVS prior. In these figures the posterior median is the solid line and the dotted lines are the $10^{t h}$ and $90^{t h}$ percentiles. These impulse responses all have sensible shapes, similar to those found by other authors. The two priors are giving similar results, but a careful examination of them do reveal some differences. Especially at longer horizons, there is evidence that SSVS leads to slightly more precise inferences (evidenced by a narrower band between the $10^{\text {th }}$ and $90^{\text {th }}$ percentiles) due to the shrinkage it provides. 


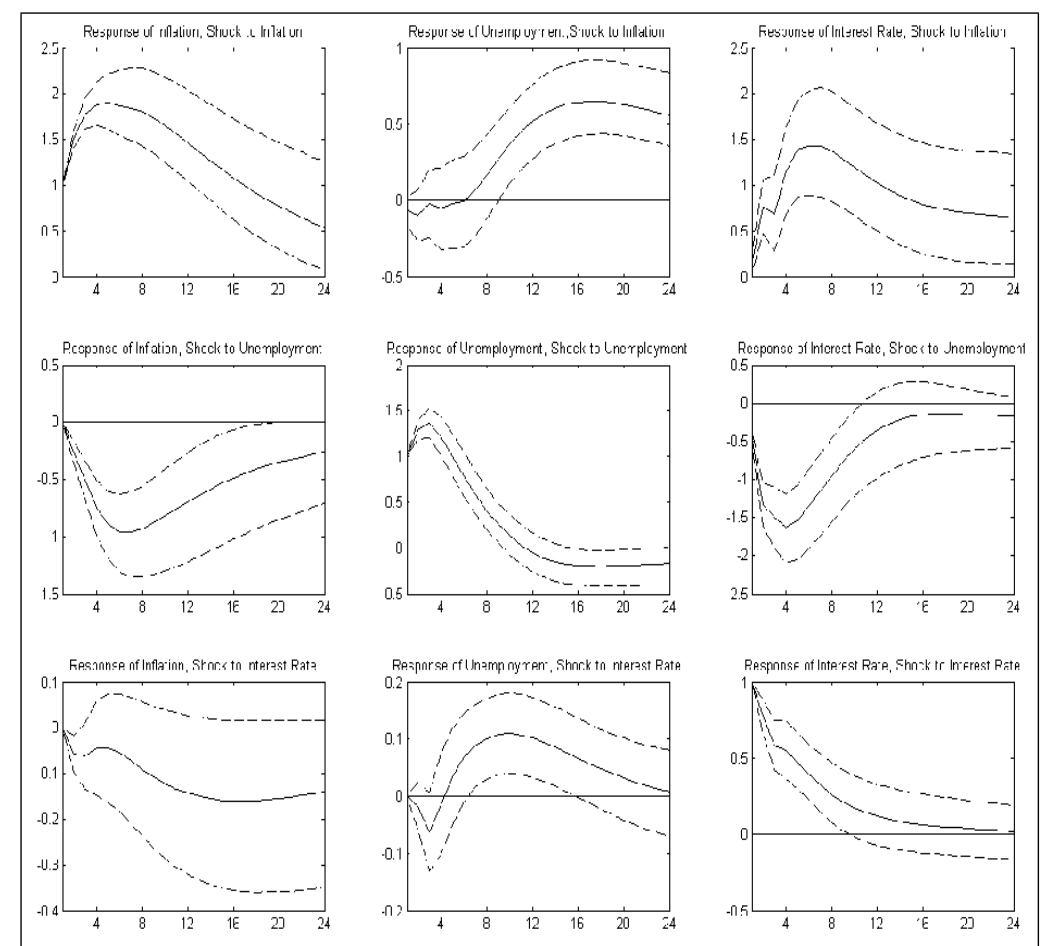

Figure 2: Posterior of impulse responses - Noninformative prior. 


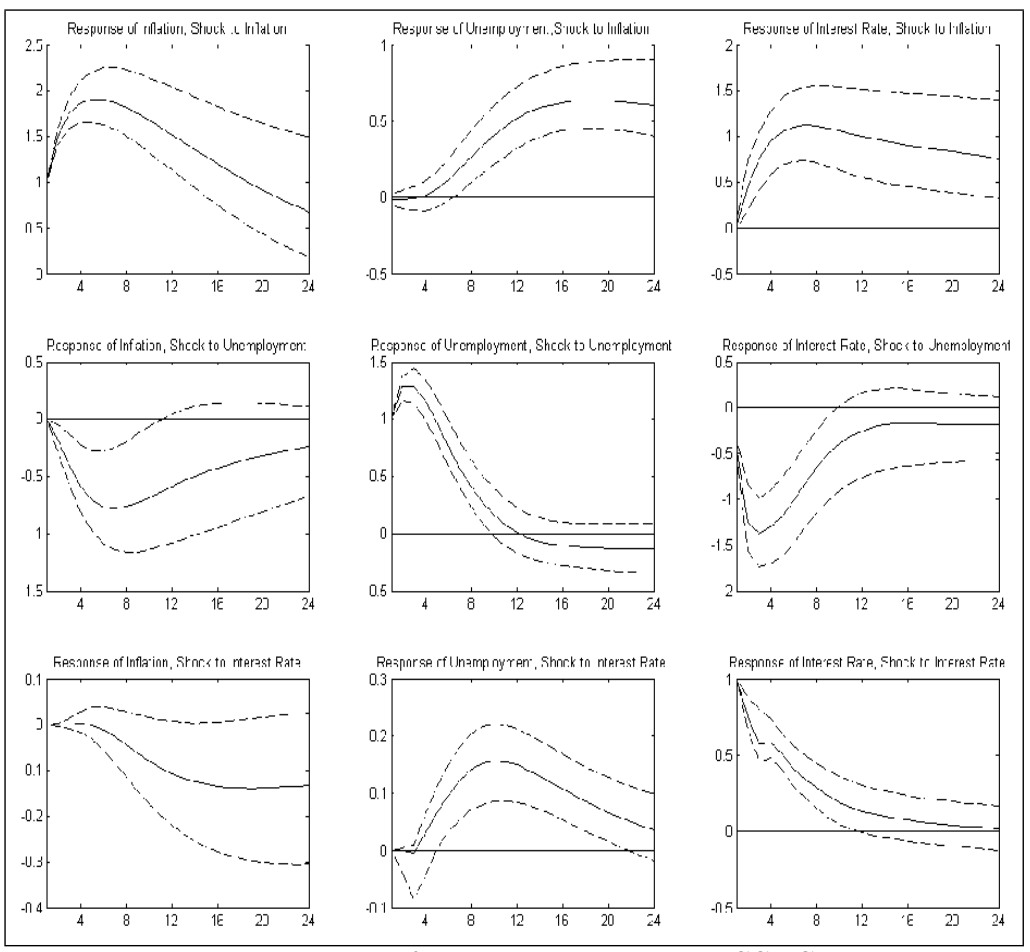

Figure 3: Posterior of impulse responses - SSVS prior.

\subsection{Empirical Illustration: Forecasting with VARs}

Our previous empirical illustration used a small VAR and focussed largely on impulse response analysis. However, VARs are commonly used for forecasting and, recently, there has been a growing interest in larger Bayesian VARs. Hence, we provide a forecasting application with a larger VAR.

Banbura, Giannone and Reichlin (2010) work with VARs with up to 130 dependent variables and find that they forecast well relative to popular alternatives such as factor models (a class of models which is discussed below). They use a Minnesota prior. Koop (2010) carries out a similar forecasting exercise, with a wider range of priors and wider range of VARs of different dimensions. Note that a 20-variate $\operatorname{VAR}(4)$ with quarterly data would have $M=20$ and $p=4$ in which case $\alpha$ contains over 1500 coefficients. $\Sigma$, too, will be parameter rich, containing over 200 distinct elements. A typical macroeconomic quarterly data set might have approximately two hundred observations and, hence, the number of coefficients will far exceed the number of observations. But Bayesian methods combine likelihood function with prior. It is well-known that, even if some parameters are not identified in the likelihood function, under weak conditions the use of a proper prior will lead to a valid posterior density and, thus, 
Bayesian inference is possible. However, prior information becomes increasingly important as the number of parameters increases relative to sample size.

The present empirical illustration uses the same US quarterly data set as Koop (2010). ${ }^{10}$ The data runs from 1959Q1 through 2008Q4. We consider small VARs with three dependent variables $(M=3)$ and larger VARs which contain the same three dependent variables plus 17 more $(M=20)$. All our VARs have four lags. For brevity, we do not provide a precise list of variables or data definitions (see Koop, 2010, for complete details). Here we note only the three main variables used with both VARs are the ones we are interested in forecasting. These are a measure of economic activity (GDP, real GDP), prices (CPI, the consumer price index) and an interest rate (FFR, the Fed funds rate). The remaining 17 variables used with the larger VAR are other common macroeconomic variables which are thought to potentially be of some use for forecasting the main three variables.

Following Stock and Watson (2008) and many others, the variables are all transformed to stationarity (usually by differencing or log differencing). With data transformed in this way, we set the prior means for all coefficients in all approaches to zero (instead of setting some prior means to one so as to shrink towards a random walk as might be appropriate if we were working with untransformed variables). We consider three priors: a Minnesota prior as implemented by Banbura, Giannone and Reichlin (2010), an SSVS prior as implemented in George, Sun and Ni (2008) as well as a prior which combines the Minnesota prior with the SSVS prior. This final prior is identical to the SSVS prior with one exception. To explain the one exception, remember that the SSVS prior involves setting the diagonal elements of the prior covariance matrix in (22) to be $\kappa_{0 j}=c_{0} \sqrt{\widehat{\operatorname{var}}\left(\alpha_{j}\right)}$ and $\kappa_{1 j}=c_{1} \sqrt{\widehat{\operatorname{var}}\left(\alpha_{j}\right)}$ where $\widehat{\operatorname{var}}\left(\alpha_{j}\right)$ is based on a posterior or OLS estimate. In our final prior, we set $\widehat{\operatorname{var}}\left(\alpha_{j}\right)$ to be the prior variance of $\alpha_{j}$ from the Minnesota prior. Other details of implementation (e.g. the remaining prior hyperparameter choices not specified here) are as in Koop (2010). Suffice it to note here that they are the same as those used in Banbura, Giannone and Reichlin (2010) and George, Sun and Ni (2008).

Previously we have shown how to obtain predictive densities using these priors. We also need a way of evaluating forecast performance. Here we consider two measures of forecast performance: one is based on point forecasts, the other involves entire predictive densities.

We carry out a recursive forecasting exercise using the direct method. That is, for $\tau=\tau_{0}, . ., T-h$, we obtain the predictive density of $y_{\tau+h}$ using data available through time $\tau$ for $h=1$ and 4. $\tau_{0}$ is 1969Q4. In this forecasting section, we will use notation where $y_{i, \tau+h}$ is a random variable we are wishing to forecast (e.g. GDP, CPI or FFR), $y_{i . \tau+h}^{o}$ is the observed value of $y_{i, \tau+h}$ and $p\left(y_{i, \tau+h} \mid D a t a_{\tau}\right)$ is the predictive density based on information available at time $\tau$.

Mean square forecast error, MSFE, is the most common measure of forecast

\footnotetext{
${ }^{10}$ This is an updated version of the data set used in Stock and Watson (2008). We would like to thank Mark Watson for making this data available.
} 
comparison. It is defined as:

$$
M S F E=\frac{\sum_{\tau=\tau_{0}}^{T-h}\left[y_{i, \tau+h}^{o}-E\left(y_{i, \tau+h} \mid D a t a_{\tau}\right)\right]^{2}}{T-h-\tau_{0}+1} .
$$

In Table 4, MSFE is presented as a proportion of the MSFE produced by random walk forecasts.

MSFE only uses the point forecasts and ignores the rest of the predictive distribution. Predictive likelihoods evaluate the forecasting performance of the entire predictive density. Predictive likelihoods are motivated and described in many places such as Geweke and Amisano (2010). The predictive likelihood is the predictive density for $y_{i, \tau+h}$ evaluated at the actual outcome $y_{i, \tau+h}^{o}$. The sum of log predictive likelihoods can be used for forecast evaluation:

$$
\sum_{\tau=\tau_{0}}^{T-h} \log \left[p\left(y_{i, \tau+h}=y_{i, \tau+h}^{o} \mid D^{\circ} a t a_{\tau}\right)\right] .
$$

Table 4 presents MSFEs and sums of log predictive likelihoods for our three main variables of interest for forecast horizons one quarter and one year in the future. All of the Bayesian VARs forecast substantially better than a random walk for all variables. However, beyond that it is hard to draw any general conclusion from Table 4. Each prior does well for some variable and/or forecast horizon. However, it is often the case that the prior which combines SSVS features with Minnesota prior features forecasts best. Note that in the larger VAR the SSVS prior can occasionally perform poorly. This is because coefficients which are included (i.e. have $\gamma_{j}=1$ ) are not shrunk to any appreciable degree. This lack of shrinkage can sometimes lead to a worsening of forecast performance. By combining the Minnesota prior with the SSVS prior we can surmount this problem.

It can also be seen that usually (but not always), the larger VARs forecast better than the small VAR. If we look only at the small VARs, the SSVS prior often leads to the best forecast performance.

Our two forecast metrics, MSFEs and sums of log predictive likelihoods, generally point towards the same conclusion. But there are exceptions where sums of log predictive likelihoods (the preferred Bayesian forecast metric) can paint a different picture than MSFEs. 


\begin{tabular}{|c|c|c|c|c|c|c|}
\hline \multirow[t]{2}{*}{ Variable } & \multicolumn{2}{|c|}{ Minnesota Prior } & \multicolumn{2}{|l|}{ SSVS Prior } & \multicolumn{2}{|c|}{ SSVS+Minnesota } \\
\hline & $M=3$ & $M=20$ & $M=3$ & $M=20$ & $M=3$ & $M=20$ \\
\hline & \multicolumn{6}{|c|}{ Forecast Horizon of One Quarter } \\
\hline GDP & $\begin{array}{l}0.650 \\
(-206.4)\end{array}$ & $\begin{array}{l}0.552 \\
(-192.3)\end{array}$ & $\begin{array}{l}0.606 \\
(-198.40)\end{array}$ & $\begin{array}{l}0.641 \\
(-205.1)\end{array}$ & $\begin{array}{l}0.698 \\
(-204.7)\end{array}$ & $\begin{array}{l}0.647 \\
(-203.9)\end{array}$ \\
\hline CPI & $\begin{array}{l}0.347 \\
(-201.2)\end{array}$ & $\begin{array}{l}0.303 \\
(-195.9)\end{array}$ & $\begin{array}{l}0.320 \\
(-193.9)\end{array}$ & $\begin{array}{l}0.316 \\
(-196.5)\end{array}$ & $\begin{array}{l}0.325 \\
(-191.5)\end{array}$ & $\begin{array}{l}0.291 \\
(-187.6)\end{array}$ \\
\hline FFR & $\begin{array}{l}0.619 \\
(-238.4)\end{array}$ & $\begin{array}{l}0.514 \\
(-229.1)\end{array}$ & $\begin{array}{l}0.844 \\
(-252.4)\end{array}$ & $\begin{array}{l}0.579 \\
(-237.2)\end{array}$ & $\begin{array}{l}0.744 \\
(-252.7)\end{array}$ & $\begin{array}{l}0.543 \\
(-228.9)\end{array}$ \\
\hline & \multicolumn{6}{|c|}{ Forecast Horizon of One Year } \\
\hline GDP & $\begin{array}{l}0.744 \\
(-220.6)\end{array}$ & $\begin{array}{l}0.609 \\
(-214.7)\end{array}$ & $\begin{array}{l}0.615 \\
(-207.8)\end{array}$ & $\begin{array}{l}0.754 \\
(-293.2)\end{array}$ & $\begin{array}{l}0.844 \\
(-221.6)\end{array}$ & $\begin{array}{l}0.667 \\
(-219.0)\end{array}$ \\
\hline CPI & $\begin{array}{l}0.525 \\
(-209.5) \\
\end{array}$ & $\begin{array}{l}0.522 \\
(-219.4) \\
\end{array}$ & $\begin{array}{l}0.501 \\
(-208.3) \\
\end{array}$ & $\begin{array}{l}0.772 \\
(-276.4) \\
\end{array}$ & $\begin{array}{l}0.468 \\
(-194.4) \\
\end{array}$ & $\begin{array}{l}0.489 \\
(-201.6) \\
\end{array}$ \\
\hline FFR & $\begin{array}{l}0.668 \\
(-243.3)\end{array}$ & $\begin{array}{l}0.587 \\
(-249.6)\end{array}$ & $\begin{array}{l}0.527 \\
(-231.2)\end{array}$ & $\begin{array}{l}0.881 \\
(-268.1)\end{array}$ & $\begin{array}{l}0.618 \\
(-228.8)\end{array}$ & $\begin{array}{l}0.518 \\
(-233.7)\end{array}$ \\
\hline
\end{tabular}

\section{Bayesian State Space Modeling and Stochas- tic Volatility}

\subsection{Introduction and Notation}

In the section on Bayesian VAR modeling, we showed that the (possibly restricted) VAR could be written as:

$$
y_{t}=Z_{t} \beta+\varepsilon
$$

for appropriate definitions of $Z_{t}$ and $\beta$. In many macroeconomic applications, it is undesirable to assume $\beta$ to be constant, but it is sensible to assume that $\beta$ evolves gradually over time. A standard version of the TVP-VAR which will be discussed in the next section extends the VAR to:

$$
y_{t}=Z_{t} \beta_{t}+\varepsilon_{t}
$$

where

$$
\beta_{t+1}=\beta_{t}+u_{t}
$$

Thus, the VAR coefficients are allowed to vary gradually over time. This is a state space model.

Furthermore, previously we assumed $\varepsilon_{t}$ to be i.i.d. $N(0, \Sigma)$ and, thus, the model was homoskedastic. In empirical macroeconomics, it is often important to allow for the error covariance matrix to change over time (e.g. due to the Great Moderation of the business cycle) and, in such cases, it is desirable to 
assume $\varepsilon_{t}$ to be i.i.d. $N\left(0, \Sigma_{t}\right)$ so as to allow for heteroskedasticity. This raises the issue of stochastic volatility which, as we shall see, also leads us into the world of state space models.

These considerations provide a motivation for why we must provide a section on state space models before proceeding to TVP-VARs and other models of more direct relevance for empirical macroeconomics. We begin this section by first discussing Bayesian methods for the Normal linear state space model. These methods can be used to model evolution of the VAR coefficients in the TVPVAR. Unfortunately, stochastic volatility cannot be written in the form of a Normal linear state space model. Thus, after briefly discussing nonlinear state space modelling in general, we present Bayesian methods for particular nonlinear state space models of interest involving stochastic volatility.

We will adopt a notational convention commonly used in the state space literature where, if $a_{t}$ is a time $t$ quantity (i.e. a vector of states or data) then $a^{t}=\left(a_{1}^{\prime}, . ., a_{t}^{\prime}\right)^{\prime}$ stacks all the $a_{t} \mathrm{~s}$ up to time $t$. So, for instance, $y^{T}$ will denote the entire sample of data on the dependent variables and $\beta^{T}$ the vector containing all the states.

\subsection{The Normal Linear State Space Model}

A general formulation for the Normal linear state space model (which contains the TVP-VAR defined above as a special case) is:

$$
y_{t}=W_{t} \delta+Z_{t} \beta_{t}+\varepsilon_{t}
$$

and

$$
\beta_{t+1}=\Pi_{t} \beta_{t}+u_{t}
$$

where $y_{t}$ is an $M \times 1$ vector containing observations on $M$ time series variables, $\varepsilon_{t}$ is an $M \times 1$ vector of errors, $W_{t}$ is a known $M \times p_{0}$ matrix (e.g. this could contain lagged dependent variables or other explanatory variables with constant coefficients), $\delta$ is a $p_{0} \times 1$ vector of parameters. $Z_{t}$ is a known $M \times k$ matrix (e.g. this could contain lagged dependent variables or other explanatory variables with time varying coefficients), $\beta_{t}$ is a $k \times 1$ vector of parameters which evolve over time (these are known as states). We assume $\varepsilon_{t}$ to be independent $N\left(0, \Sigma_{t}\right)$ and $u_{t}$ to be a $k \times 1$ vector which is independent $N\left(0, Q_{t}\right) . \varepsilon_{t}$ and $u_{s}$ are independent of one another for all $s$ and $t$. $\Pi_{t}$ is a $k \times k$ matrix which is typically treated as known, but occasionally $\Pi_{t}$ is treated as a matrix of unknown parameters.

Equations (28) and (29) define a state space model. Equation (28) is called the measurement equation and (29) the state equation. Models such as this have been used for a wide variety of purposes in econometrics and many other fields. The interested reader is referred to West and Harrison (1997) and Kim and Nelson (1999) for a broader Bayesian treatment of state space models than that provided here. Harvey (1989) and Durbin and Koopman (2001) provide good non-Bayesian treatments of state space models. 
For our purpose, the important thing to note is that, for given values of $\delta, \Pi_{t}$, $\Sigma_{t}$ and $Q_{t}$ (for $t=1, . ., T$ ), various algorithms have been developed which allow for posterior simulation of $\beta_{t}$ for $t=1, . ., T$. Popular and efficient algorithms are described in Carter and Kohn (1994), Fruhwirth-Schnatter (1994), DeJong and Shephard (1995) and Durbin and Koopman (2002). ${ }^{11}$ Since these are standard and well-understood algorithms, we will not present complete details here. In the Matlab code on the website associated with this monograph, the algorithm of Carter and Kohn (1994) is used. These algorithms can be used as a block in an MCMC algorithm to provide draws from the posterior of $\beta_{t}$ conditional on $\delta, \Pi_{t}, \Sigma_{t}$ and $Q_{t}($ for $t=1, . ., T)$. The exact treatment of $\delta, \Pi_{t}, \Sigma_{t}$ and $Q_{t}$ depends on the empirical application at hand. The standard TVP-VAR fixes some of these to known values (e.g. $\delta=0, \Pi_{t}=I$ are common choices) and treats others as unknown parameters (although it usually restricts $Q_{t}=Q$ and, in the case of the homoskedastic TVP-VAR additionally restricts $\Sigma_{t}=\Sigma$ for all $t$ ). An MCMC algorithm is completed by taking draws of the unknown parameters from their posteriors (conditional on the states). The next part of this section elaborates on how this MCMC algorithm works. To focus on state space model issues, the algorithm is for the case where $\delta$ is a vector of unknown parameters, $Q_{t}=Q$ and $\Sigma_{t}=\Sigma$ and $\Pi_{t}$ is known.

An examination of (28) reveals that, if $\beta_{t}$ for $t=1, . ., T$ were known (as opposed to being unobserved), then the state space model would reduce to a multivariate Normal linear regression model:

$$
y_{t}^{*}=W_{t} \delta+\varepsilon_{t},
$$

where $y_{t}^{*}=y_{t}-Z_{t} \beta_{t}$. Thus, standard results for the multivariate Normal linear regression model could be used, except the dependent variable would be $y_{t}^{*}$ instead of $y_{t}$. This suggests that an MCMC algorithm can be set up for the state space model. That is, $p\left(\delta \mid y^{T}, \Sigma, \beta^{T}\right)$ and $p\left(\Sigma^{-1} \mid y^{T}, \delta, \beta^{T}\right)$ will typically have a simple textbook form. Below we will use the independent Normal-Wishart prior for $\delta$ and $\Sigma^{-1}$. This was introduced in our earlier discussion of VAR models.

Note next that a similar reasoning can be used for the covariance matrix for the error in the state equation. That is, if $\beta_{t}$ for $t=1, . ., T$ were known, then the state equation, (29), is a simple variant of multivariate Normal regression model. This line of reasoning suggests that $p\left(Q^{-1} \mid y^{T}, \delta, \beta^{T}\right)$ will have a simple and familiar form. ${ }^{12}$

\footnotetext{
${ }^{11}$ Each of these algorithms has advantages and disadvantages. For instance, the algorithm of DeJong and Shephard (1995) works well with degenerate states. Recently some new algorithms have been proposed which do not involve the use of Kalman filtering or simulation smoothing. These methods show great promise, see McCausland, Miller and Pelletier (2007) and Chan and Jeliazkov (2009).

${ }^{12}$ The case where $\Pi_{t}$ contains unknown parameters would involve drawing from $p\left(Q, \Pi_{1}, . ., \Pi_{T} \mid y, \beta_{1}, . ., \beta_{T}\right)$ which can usually be done fairly easily. In the time-invariant case where $\Pi_{1}=. .=\Pi_{T} \equiv \Pi, p\left(\Pi, Q \mid y, \beta_{1}, . ., \beta_{T}\right)$ has a form of the same structure as a VAR.
} 
Combining these results for $p\left(\delta \mid y^{T}, \Sigma, \beta^{T}\right), p\left(\Sigma^{-1} \mid y^{T}, \delta, \beta^{T}\right)$ and $p\left(Q^{-1} \mid y^{T}, \delta, \beta^{T}\right)$ with one of the standard methods (e.g. that of Carter and Kohn, 1994) for taking random draws from $p\left(\beta^{T} \mid y^{T}, \delta, \Sigma, Q\right)$ will completely specify an MCMC algorithm which allows for Bayesian inference in the state space model. In the following material we develop such an MCMC algorithm for a particular prior choice, but we stress that other priors can be used with minor modifications.

Here we will use an independent Normal-Wishart prior for $\delta$ and $\Sigma^{-1}$ and a Wishart prior for $Q^{-1}$. It is worth noting that the state equation can be interpreted as already providing us with a prior for $\beta^{T}$. That is, (29) implies:

$$
\beta_{t+1} \mid \beta_{t}, Q \sim N\left(\Pi_{t} \beta_{t}, Q\right) .
$$

Formally, the state equation implies the prior for the states is:

$$
p\left(\beta^{T} \mid Q\right)=\prod_{t=1}^{T} p\left(\beta_{t} \mid \beta_{t-1}, Q\right)
$$

where the terms on the right-hand side are given by (30). This is an example of a hierarchical prior, since the prior for $\beta^{T}$ depends on the $Q$ which, in turn, requires its own prior.

One minor issue should be mentioned: that of initial conditions. The prior for $\beta_{1}$ depends on $\beta_{0}$. There are standard ways of treating this issue. For instance, if we assume $\beta_{0}=0$, then the prior for $\beta_{1}$ becomes:

$$
\beta_{1} \mid Q \sim N(0, Q) .
$$

Similarly, authors such as Carter and Kohn (1994) simply assume $\beta_{0}$ has some unspecified distribution as its prior. Alternatively, in the TVP-VAR (or any TVP regression model) we can simply set $\beta_{1}=0$ and $W_{t}=Z_{t} \cdot{ }^{13}$

Combining these prior assumptions together, we have

$$
p\left(\delta, \Sigma, Q, \beta^{T}\right)=p(\delta) p(\Sigma) p(Q) p\left(\beta^{T} \mid Q\right)
$$

where

$$
\begin{gathered}
\delta \sim N(\underline{\delta}, \underline{V}), \\
\Sigma^{-1} \sim W\left(\underline{S}^{-1}, \underline{\nu}\right),
\end{gathered}
$$

and

$$
Q^{-1} \sim W\left(\underline{Q}^{-1}, \underline{\nu}_{Q}\right) .
$$

The reasoning above suggests that our end goal is an MCMC algorithm which sequentially draws from $p\left(\delta \mid y^{T}, \Sigma, \beta^{T}\right), p\left(\Sigma^{-1} \mid y^{T}, \delta, \beta^{T}\right), p\left(Q^{-1} \mid y^{T}, \delta, \beta^{T}\right)$

\footnotetext{
${ }^{13}$ This result follows from the fact that $y_{t}=Z_{t} \beta_{t}+\varepsilon_{t}$ with $\beta_{1}$ left unrestricted and $y_{t}=$ $Z_{t} \delta+Z_{t} \beta_{t}+\varepsilon_{t}$ with $\beta_{1}=0$ are equivalent models.
} 
and $p\left(\beta^{T} \mid y^{T}, \delta, \Sigma, Q\right)$. The first three of these posterior conditional distributions can be dealt with by using results for the multivariate Normal linear regression model. In particular,

$$
\delta \mid y^{T}, \Sigma, \beta^{T} \sim N(\bar{\delta}, \bar{V}) .
$$

where

$$
\bar{V}=\left(\underline{V}^{-1}+\sum_{t=1}^{T} W_{t}^{\prime} \Sigma^{-1} W_{t}\right)^{-1}
$$

and

$$
\bar{\delta}=\bar{V}\left(\underline{V}^{-1} \underline{\delta}+\sum_{t=1}^{T} W_{t}^{\prime} \Sigma^{-1}\left(y_{t}-Z_{t} \beta_{t}\right)\right) .
$$

Next we have

$$
\Sigma^{-1} \mid y^{T}, \delta, \beta^{T} \sim W\left(\bar{S}^{-1}, \bar{\nu}\right)
$$

where

$$
\bar{\nu}=T+\underline{\nu}
$$

and

$$
\bar{S}=\underline{S}+\sum_{t=1}^{T}\left(y_{t}-W_{t} \delta-Z_{t} \beta_{t}\right)\left(y_{t}-W_{t} \delta-Z_{t} \beta_{t}\right)^{\prime} .
$$

Next,

$$
Q^{-1} \mid y^{T}, \delta, \beta^{T} \sim W\left(\bar{Q}^{-1}, \bar{\nu}_{Q}\right)
$$

where

$$
\bar{\nu}_{Q}=T+\underline{\nu}_{Q}
$$

and

$$
\bar{Q}=\underline{Q}+\sum_{t=1}^{T}\left(\beta_{t+1}-\Pi_{t} \beta_{t}\right)\left(\beta_{t+1}-\Pi_{t} \beta_{t}\right)^{\prime} .
$$

To complete our MCMC algorithm, we need a means of drawing from $p\left(\beta^{T} \mid y^{T}, \delta, \Sigma, Q\right)$. But, as discussed previously, there are several standard algorithms that can be used for doing this. Accordingly, Bayesian inference in the Normal linear state space model can be done in a straightforward fashion. We will draw on these results when we return to the TVP-VAR in a succeeding section of this monograph. 


\subsection{Nonlinear State Space Models}

The Normal linear state space model discussed previously is used by empirical macroeconomists not only when working with TVP-VARs, but also for many other purposes. For instance, Bayesian analysis of DSGE models has become increasingly popular (see, e.g., An and Schorfheide, 2007 or Fernandes-Villaverde, 2009). Estimation of linearized DSGE models involves working with the Normal linear state space model and, thus, the methods discussed above can be used. However, linearizing of DSGE models is done through first order approximations and, very recently, macroeconomists have expressed an interest in using second order approximations. When this is done the state space model becomes nonlinear (in the sense that the measurement equation has $y_{t}$ being a nonlinear function of the states). This is just one example of how nonlinear state space models can arise in macroeconomics. There are an increasing number of tools which allow for Bayesian computation in nonlinear state space models (e.g. the particle filter is enjoying increasing popularity see, e.g., Johannes and Polson, 2009). Given the focus of this monograph on TVP-VARs and related models, we will not offer a general discussion of Bayesian methods for nonlinear state space models (see Del Negro and Schorfheide, 2010, and Giordani, Pitt and Kohn, 2010 for further discussion). Instead we will focus on an area of particular interest for the TVP-VAR modeler: stochastic volatility.

Broadly speaking, issues relating to the volatility of errors have obtained an increasing prominence in macroeconomics. This is due partially to the empirical regularities that are often referred to as the Great Moderation of the business cycle (i.e. that the volatilities of many macroeconomic variables dropped in the early 1980s and remained low until recently). But it is also partly due to the fact that many issues of macroeconomic policy hinge on error variances. For instance, the debate on why the Great Moderation occurred is often framed in terms of "good policy" versus "good luck" stories which involve proper modeling of error variances. For these reasons, volatility is important so we will spend some time describing Bayesian methods for handling it.

\subsubsection{Univariate Stochastic Volatility}

We begin with a discussion of stochastic volatility when $y_{t}$ is a scalar. Although TVP-VARs are multivariate in nature and, thus, Bayesian methods for multivariate stochastic volatility are required, these use methods for univariate stochastic volatility as building blocks. Accordingly, a Bayesian treatment of univariate stochastic volatility is a useful starting point. In order to focus the discussion, we will assume there are no explanatory variables and, hence, adopt a simple univariate stochastic volatility model $^{14}$ which can be written as:

\footnotetext{
${ }^{14}$ In this section we describe a method developed in Kim, Shephard and Chib (1998) which has become more popular than the pioneering approach of Jacquier, Polson and Rossi (1994). Bayesian methods for extensions of this standard stochastic volatility model (e.g. involving non-Normal errors or leverage effects) can be found in Chib, Nardari and Shephard (2002) and Omori, Chib, Shephard and Nakajima (2007).
} 


$$
y_{t}=\exp \left(\frac{h_{t}}{2}\right) \varepsilon_{t}
$$

and

$$
h_{t+1}=\mu+\phi\left(h_{t}-\mu\right)+\eta_{t},
$$

where $\varepsilon_{t}$ is i.i.d. $N(0,1)$ and $\eta_{t}$ is i.i.d. $N\left(0, \sigma_{\eta}^{2}\right) \cdot \varepsilon_{t}$ and $\eta_{s}$ are independent of one another for all $s$ and $t$.

Note that (34) and (35) is a state space model similar to (28) and (29) where $h_{t}$ for $t=1, . ., t$ can be interpreted as states. However, in contrast to (28), (34) is not a linear function of the states and, hence, our results for Normal linear state space models cannot be directly used.

Note that this parameterization is such that $h_{t}$ is the log of the variance of $y_{t}$. Since variances must be positive, in order to sensibly have Normal errors in the state equation (35), we must define the state equation as holding for $\log$-volatilities. Note also that $\mu$ is the unconditional mean of $h_{t}$.

With regards to initial conditions, it is common to restrict the log-volatility process to be stationary and impose $|\phi|<1$. Under this assumption, it is sensible to have:

$$
h_{0} \sim N\left(\mu, \frac{\sigma_{\eta}^{2}}{1-\phi^{2}}\right)
$$

and the algorithm of Kim, Shephard and Chib (1998) described below uses this specification. However, in the TVP-VAR literature it is common to have VAR coefficients evolving according to random walks and, by analogy, TVP-VAR papers such as Primiceri (2005) often work with (multivariate extensions of) random walk specifications for the log-volatilities and set $\phi=1$. This simplifies the model since, not only do parameters akin to $\phi$ not have to be estimated, but also $\mu$ drops out of the model. However, when $\phi=1$, the treatment of the initial condition given in (36) cannot be used. In this case, a prior such as $h_{0} \sim N\left(\underline{h}, \underline{V}_{h}\right)$ is typically used. This requires the researcher to choose $\underline{h}$ and $\underline{V}_{h}$. This can be done subjectively or, as in Primiceri (2005), an initial "training sample" of the data can be set aside to calibrate values for the prior hyperparameters.

In the development of an MCMC algorithm for the stochastic volatility model, the key part is working out how to draw the states. That is (in a similar fashion as for the parameters in the Normal linear state space model), $p\left(\phi \mid y^{T}, \mu, \sigma_{\eta}^{2}, h^{T}\right), p\left(\mu \mid y^{T}, \phi, \sigma_{\eta}^{2}, h^{T}\right)$ and $p\left(\sigma_{\eta}^{2} \mid y^{T}, \mu, \phi, h^{T}\right)$ have standard forms derived using textbook results for the Normal linear regression model and will not be presented here (see, e.g., Kim, Shephard and Chib, 1998 for exact formulae). To complete an MCMC algorithm, all that we require is a method for taking draws from $p\left(h^{T} \mid y^{T}, \mu, \phi, \sigma_{\eta}^{2}\right)$. Kim, Shephard and Chib (1998) provide an efficient method for doing this. To explain the basic ideas underlying this algorithm, note that if we square both sides of the measurement equation, (34), and then take logs we obtain: 


$$
y_{t}^{*}=h_{t}+\varepsilon_{t}^{*}
$$

where $^{15} y_{t}^{*}=\ln \left(y_{t}^{2}\right)$ and $\varepsilon_{t}^{*}=\ln \left(\varepsilon_{t}^{2}\right)$. Equations (37) and (35) define a state space model which is linear in the states. The only thing which prevents us from immediately using our previous results for the Normal linear state space model is the fact that $\varepsilon_{t}^{*}$ is not Normal. However, as well shall see, it can be approximated by a mixture of different Normal distributions and this allows us to exploit our earlier results.

Mixtures of Normal distributions are very flexible and have been used widely in many fields to approximate unknown or inconvenient distributions. In the case of stochastic volatility, Kim, Shephard and Chib (1998) show that the distribution of $\varepsilon_{t}^{*}, p\left(\varepsilon_{t}^{*}\right)$ can be well-approximated by:

$$
p\left(\varepsilon_{t}^{*}\right) \approx \sum_{i=1}^{7} q_{i} f_{N}\left(\varepsilon_{t}^{*} \mid m_{i}, v_{i}^{2}\right),
$$

where $f_{N}\left(\varepsilon_{t}^{*} \mid m_{i}, v_{i}^{2}\right)$ is the p.d.f. of a $N\left(m_{i}, v_{i}^{2}\right)$ random variable. ${ }^{16}$ Crucially, since $\varepsilon_{t}$ is $N(0,1)$ it follows that $\varepsilon_{t}^{*}$ involves no unknown parameters and neither does this approximation. Thus, $q_{i}, m_{i}, v_{i}^{2}$ for $i=1, . ., 7$ are not parameters to be estimated, but simply numbers given in Table 4 of Kim, Shephard and Chib (1998).

An equivalent way of writing (38) is to introduce component indicator variables, $s_{t} \in\{1,2, . ., 7\}$ for each element in the Normal mixture and writing:

$$
\begin{aligned}
& \varepsilon_{t}^{*} \mid s_{t}=i \sim N\left(m_{i}, v_{i}^{2}\right) \\
& \operatorname{Pr}\left(s_{t}=i\right)=q_{i}
\end{aligned},
$$

for $i=1, . ., 7$. This formulation provides insight into how the algorithm works. In particular, the MCMC algorithm does not simply draw the log-volatilities from $p\left(h^{T} \mid y^{T}, \mu, \phi, \sigma_{\eta}^{2}\right)$, but rather draws them from $p\left(h^{T} \mid y^{T}, \mu, \phi, \sigma_{\eta}^{2}, s^{T}\right)$. This may seem awkward, but has the huge benefit that standard results from the Normal linear state space models such as those described previously in this section can be used. That is, conditional on knowing $s_{1}, . ., s_{T}$, the algorithm knows which of the seven Normals $\varepsilon_{t}^{*}$ comes from at each $t=1, . ., T$ and the model becomes a Normal linear state space model. To complete the MCMC algorithm requires a method for drawing from $p\left(s^{T} \mid y^{T}, \mu, \phi, \sigma_{\eta}^{2}, h^{T}\right)$ but this is simple to do since $s_{t}$ is a discrete distribution with seven points of support. Precise details are given in Kim, Shephard and Chib (1998).

A Digression: Marginal Likelihood Calculation in State Space Models Marginal likelihoods are the most popular tool for Bayesian model comparison

\footnotetext{
${ }^{15}$ In practice, it is common to set $y_{t}^{*}=\ln \left(y_{t}^{2}+c\right)$ where $c$ is known as an off-set constant set to a small number (e.g. $c=0.001$ ) to avoid numerical problems associated with times where $y_{t}^{2}$ is zero or nearly so.

${ }^{16}$ Omori, Chib, Shephard and Nakajima (2007) recommend an even more accurate approximation using a mixture of 10 Normal distributions.
} 
(e.g. Bayes factors are ratios of marginal likelihoods). In this monograph, we focus on estimation and prediction as opposed to model comparison or hypothesis testing. This is partly because state space models such as TVP-VARs are very flexible and can approximate a wide range of data features. Thus, many researchers prefer to treat them as being similar in spirit to nonparametric models: capable of letting the data speak and uncovering an appropriate model (as opposed to working with several parsimonious models and using statistical methods to select a single one). Furthermore, a Bayesian rule of thumb is that the choice of prior matters much less for estimation and prediction than it does for marginal likelihoods. This is particularly true for high-dimensional models such as TVP-VARs where marginal likelihoods can be sensitive to the choice of prior. For this reason, many Bayesians avoid the use of marginal likelihoods in high dimensional models. Even those who wish to do model comparison often use other metrics (e.g. Geweke and Keane, 2007, uses cross-validation and Geweke, 1996, uses predictive likelihoods).

However, for the researcher who wishes to use marginal likelihoods, note that there are many methods for calculating them that involve the evaluation of the likelihood function at a point. For instance, information criteria are often approximations to marginal likelihoods and these involve calculating the maximum likelihood estimator. Popular methods of marginal likelihood calculation such as Chib (1995), Chib and Jeliazkov (2001, 2005) and Gelfand and Dey (1994) involve evaluating the likelihood function. In state space models, a question arises as to which likelihood function should be used. In terms of our notation for the Normal linear state space model, $p\left(y^{T} \mid \delta, \Sigma, Q, \beta^{T}\right)$ and $p\left(y^{T} \mid \delta, \Sigma, Q\right)$ can both be used as "likelihoods" and either could be used in any of the methods of marginal likelihood calculation just cited. ${ }^{17}$ However, using $p\left(y^{T} \mid \delta, \Sigma, Q, \beta^{T}\right)$ to define the likelihood function could potentially lead to very inefficient computation since the parameter space is of such high dimension. ${ }^{18}$ Thus, it is desirable to use $p\left(y^{T} \mid \delta, \Sigma, Q\right)$ to define the likelihood function. Fortunately, for the Normal linear state space model a formula for $p\left(y^{T} \mid \delta, \Sigma, Q\right)$ is available which can be found in textbooks such as Harvey (1989) or Durbin and Koopman (2001). Chan and Jeliazkov (2009) provide an alternative algorithm for calculating the marginal likelihood in the Normal linear state space model.

For the stochastic volatility model, for the same reasons either $p\left(y^{T} \mid \phi, \mu, \sigma_{\eta}^{2}, h^{T}\right)$ or $p\left(y^{T} \mid \phi, \mu, \sigma_{\eta}^{2}\right)$ could be used to define the likelihood function. It is desirable to to use $p\left(y^{T} \mid \phi, \mu, \sigma_{\eta}^{2}\right)$ but, unfortunately, an analytical expression for it does not exist. Several methods have been used to surmount this problem, but some of them can be quite complicated (e.g. involving using particle filtering methods to integrate out $h^{T}$ ). Berg, Meyer and Yu (2004) discuss these issues in detail and recommend a simple approximation called the Deviance Information

\footnotetext{
${ }^{17}$ Fruhwirth-Schnatter and Wagner (2008) refer to the former of these as the complete data likelihood and the latter as the integrated likelihood.

${ }^{18}$ The non-Bayesian seeking to find the maximum of this likelihood function would also often run into troubles optimizing in such a high dimensional parameter space.
} 
Criterion.

It is also worth noting that the MCMC algorithm for the stochastic volatility model is an example of an auxiliary mixture sampler. That is, it introduces an auxiliary set of states, $s^{T}$, which results in a mixture of Normals representation. Conditional on these states, the model is a Normal linear state space model. Fruhwirth-Schnatter and Wagner (2008) exploit this Normality (conditional on the auxiliary states) result to develop methods for calculating marginal likelihoods using auxiliary mixture samplers and such methods can be used with stochastic volatility models.

\subsubsection{Multivariate Stochastic Volatility}

Let us now return to the state space model of (28) and (29) where $y_{t}$ is an $M \times 1$ vector and $\varepsilon_{t}$ is i.i.d. $N\left(0, \Sigma_{t}\right)$. As we have stressed previously, in empirical macroeconomics it is often very important to allow for $\Sigma_{t}$ to be time varying. There are many ways of doing this. Note that $\Sigma_{t}$ is an $M \times M$ positive definite matrix with $\frac{M(M+1)}{2}$ distinct elements. Thus, the complete set of $\Sigma_{t}$ for $t=1, . ., T$ contains $\frac{T M(M+1)}{2}$ unknown parameters which is a huge number. In one sense, the literature on multivariate stochastic volatility can be thought of as mitigating this proliferation of parameters problems through parametric restrictions and/or priors and working in parameterizations which ensure that $\Sigma_{t}$ is always positive definite. Discussions of various approaches can be found in Asai, McAleer and Yu (2006), Chib, Nardari and Shephard (2006) and Chib, Omori and Asai (2009) and the reader is referred to these papers for complete treatments. In this section, we will describe two approaches popular in macroeconomics. The first was popularized by Cogley and Sargent (2005), the second by Primiceri (2005).

To focus on the issues relating to multivariate stochastic volatility, we will consider the model:

$$
y_{t}=\varepsilon_{t}
$$

and $\varepsilon_{t}$ is i.i.d. $N\left(0, \Sigma_{t}\right)$. Before discussing the specifications used by Cogley and Sargent (2005) and Primiceri (2005) for $\Sigma_{t}$ we begin with a very simple specification such that

$$
\Sigma_{t}=D_{t}
$$

where $D_{t}$ is a diagonal matrix with each diagonal element having a univariate stochastic volatility specification. That is, if $d_{i t}$ is the $i^{\text {th }}$ diagonal element of $D_{t}$ for $i=1, . ., M$, then we write $d_{i t}=\exp \left(h_{i t}\right)$ and

$$
h_{i, t+1}=\mu_{i}+\phi_{i}\left(h_{i t}-\mu_{i}\right)+\eta_{i t},
$$

where $\eta_{t}=\left(\eta_{1 t}^{\prime}, . ., \eta_{M t}^{\prime}\right)^{\prime}$ is i.i.d. $N\left(0, D_{\eta}\right)$ where $D_{\eta}$ is a diagonal matrix (so the errors in the state equation are independent of one another). This model is simple to work with in that it simply says that each error follows its own 
univariate stochastic volatility model, independent of all the other errors. Thus, the Kim, Shephard and Chib (1998) MCMC algorithm can be used one equation at a time.

This model is typically unsuitable for empirical macroeconomic research since it is not appropriate to assume $\Sigma_{t}$ to be diagonal. Many interesting macroeconomic features (e.g. impulse responses) depend on error covariances so assuming them to be zero may be misleading. Some researchers such as Cogley and Sargent (2005) allow for non-zero covariances in a simple way by writing:

$$
\Sigma_{t}=L^{-1} D_{t} L^{-1 \prime}
$$

where $D_{t}$ is a diagonal matrix with diagonal elements being the error variances and $L$ is a lower triangular matrix with ones on the diagonal. For instance, in the $M=3$ case we have

$$
L=\left[\begin{array}{lll}
1 & 0 & 0 \\
L_{21} & 1 & 0 \\
L_{31} & L_{32} & 1
\end{array}\right]
$$

This form is particularly attractive for computation since, even through $\varepsilon_{i t}$ and $\varepsilon_{j t}$ (which are the $i^{t h}$ and $j^{t h}$ elements of $\varepsilon_{t}$ ) are no longer independent of one another, we can transform (39) as

$$
L y_{t}=L \varepsilon_{t}
$$

and $\varepsilon_{t}^{*}=L \varepsilon_{t}$ will now have a diagonal covariance matrix. In the context of an MCMC algorithm involving $p\left(h^{T} \mid y^{T}, L\right)$ and $p\left(L \mid y^{T}, h^{T}\right)$ (where $h^{T}$ stacks all $h_{t}=\left(h_{1 t}^{\prime}, . ., h_{M t}^{\prime}\right)^{\prime}$ into a $M T \times 1$ vector $)$ we can exploit this result to run the Kim, Shephard and Chib (1998) algorithm one equation at a time. That is, conditional on an MCMC draw of $L$, we can transform the model as in (42) and use results for the univariate stochastic volatility model one transformed equation at a time.

Finally, to complete the MCMC algorithm for the Cogley-Sargent model we need to take draws from $p\left(L \mid y^{T}, h^{T}\right)$. But this is straightforward since (42) shows that this model can be written as a series of $M$ regression equations with Normal errors which are independent of one another. Hence, standard results for the Normal linear regression model can be used to draw from $p\left(L \mid y^{T}, h^{T}\right)$. The appendix to Cogley and Sargent (2005) provides precise formulae for the MCMC algorithm (although their paper uses a different algorithm for drawing from $p\left(h^{T} \mid y^{T}, L\right)$ than the algorithm in Kim, Shephard and Chib, 1998, discussed in this section).

It is worth stressing that the Cogley-Sargent model allows the covariance between the errors to change over time, but in a tightly restricted fashion related to the way the error variances are changing. This can be seen most clearly in the $M=2$ case where $\varepsilon_{1 t}$ and $\varepsilon_{2 t}$ are the errors in the two equations. In this, case (41) implies $\operatorname{cov}\left(\varepsilon_{1 t}, \varepsilon_{2 t}\right)=d_{1 t} L_{21}$ which varies proportionally with the error variance of the first equation. In impulse response analysis, it can be shown 
that this restriction implies that a shock to the $i^{t h}$ variable has an effect on the $j^{\text {th }}$ variable which is constant over time. In some macroeconomic applications, such a specification might be too restrictive.

Another popular approach (see, e.g., Primiceri, 2005) extends (41) to:

$$
\Sigma_{t}=L_{t}^{-1} D_{t} L_{t}^{-1 \prime}
$$

where $L_{t}$ is defined in the same way as $L$ (i.e. as being a lower-triangular matrix with ones on the diagonal), but is now time varying. This specification does not restrict the covariances and variances in $\Sigma_{t}$ in any way. The MCMC algorithm for posterior simulation from the Primiceri model is the largely same as for the model with constant $L$ (with the trivial change that the transformation in (42) becomes $\left.L_{t} y_{t}=L_{t} \varepsilon_{t}\right)$. The main change in the algorithm arises in the way $L_{t}$ is drawn.

To describe the manner in which $L_{t}$ evolves, we first stack the unrestricted elements by rows into a $\frac{M(M-1)}{2}$ vector as $l_{t}=\left(L_{21, t}, L_{31, t}, L_{32, t}, . ., L_{p(p-1), t}\right)^{\prime}$. These can be allowed to evolve according to the state equation:

$$
l_{t+1}=l_{t}+\zeta_{t},
$$

where $\zeta_{t}$ is i.i.d. $N\left(0, D_{\zeta}\right)$ and independent of the other errors in the model and $D_{\zeta}$ is a diagonal matrix.

We have seen how the measurement equation in this model can be written as:

$$
L_{t} y_{t}=\varepsilon_{t}^{*}
$$

and it can be shown that $\varepsilon_{t}^{*} \sim N\left(0, D_{t}\right)$. We can use the structure of $L_{t}$ to isolate $y_{t}$ on the left hand side and write:

$$
y_{t}=C_{t} l_{t}+\varepsilon_{t}^{*} \text {. }
$$

Primiceri (2005), page 845 gives a general definition of $C_{t}$. For $M=3$,

$$
C_{t}=\left[\begin{array}{lll}
0 & 0 & 0 \\
-y_{1 t} & 0 & 0 \\
0 & -y_{1 t} & -y_{2 t}
\end{array}\right] \text {, }
$$

where $y_{i t}$ is the $i^{\text {th }}$ element of $y_{t}$. But (44) and (45) is now in form of a Normal linear state space model of the sort we began this section with. Accordingly, in the context of an MCMC algorithm, we can draw $L_{t}$ (conditional on $h^{T}$ and all the other model parameters) using an algorithm such as that of Carter and Kohn (1994) or Durbin and Koopman (2002).

Note that we have assumed $D_{\zeta}$ to be a diagonal matrix. Even with this restriction, the resulting multivariate stochastic volatility model is very flexible. However, should the researcher wish to have $D_{\zeta}$ being non-diagonal, it is worth noting that if it is simply assumed to be a positive definite matrix then the simplicity of the MCMC algorithm (i.e. allowing for the use of methods for 
Normal linear state space models), breaks down. Primiceri (2005) assumes $D_{\zeta}$ to have a certain block diagonal structure such that it is still possible to use algorithms for Normal linear state space models to draw $L_{t}$. It is also possible to extend this model to allow for $D_{\eta}$ (the error covariance matrix in the state equation for $h_{t}$ defined after equation 40) to be any positive definite matrix (rather than the diagonal one assumed previously). Exact formulae are provided in Primiceri (2005) for both these extensions. The empirical illustration below uses these generalizations of Primiceri (2005).

\section{TVP-VARs}

VARs are excellent tools for modeling the inter-relationships between macroeconomic variables. However, they maintain the rather strong assumption that parameters are constant over time. There are many reasons for thinking such an assumption may be too restrictive in many macroeconomic applications. Consider, for instance, U.S. monetary policy and the question of whether the high inflation and slow growth of the 1970s were due to bad policy or bad luck. Some authors (e.g. Boivin and Giannoni, 2006, Cogley and Sargent, 2001 and Lubik and Schorfheide, 2004) have argued that the way the Fed reacted to inflation has changed over time (e.g. under the Volcker and Greenspan chairmanship, the Fed was more aggressive in fighting inflation pressures than under Burns). This is the "bad policy" story and is an example of a change in the monetary policy transmission mechanism. This story depends on having VAR coefficients different in the 1970s than subsequently. Others (e.g. Sims and Zha, 2006) have emphasized that the variance of the exogenous shocks has changed over time and that this alone may explain many apparent changes in monetary policy. This is the "bad luck" story (i.e. in the 1970s volatility was high, whereas later policymakers had the good fortune of the Great Moderation of the business cycle) which motivates the addition of multivariate stochastic volatility to VAR models . Yet others (e.g. Primiceri, 2005, Koop, Leon-Gonzalez and Strachan, 2009) have found that both the transmission mechanism and the variance of the exogenous shocks have changed over time.

This example is intended to motivate the basic point that an understanding of macroeconomic policy issues should be based on multivariate models where both the VAR coefficients and the error covariance matrix can potentially change over time. More broadly, there is a large literature in macroeconomics which documents structural breaks and other sorts of parameter change in many time series variables (see, among many others, Stock and Watson, 1996). A wide range of alternative specifications have been suggested, including Markov switching VARs (e.g. Paap and van Dijk, 2003, or Sims and Zha, 2006) and other regime-switching VARs (e.g. Koop and Potter, 2006). However, perhaps the most popular have been TVP-VARs. A very incomplete list of references which use TVP-VARs includes Canova (1993), Cogley and Sargent (2001, 2005), Primiceri (2005), Canova and Gambetti (2009), Canova and Ciccarelli (2009) and Koop, Leon-Gonzalez and Strachan (2009). In this monograph, we will not 
discuss regime-switching models, but rather focus on TVP-VARs.

\subsection{Homoskedastic TVP-VARs}

To discuss some basic issues with TVP-VAR modelling, we will begin with a homoskedastic version of the model (i.e. $\Sigma_{t}=\Sigma$ ). We will use the same definition of the dependent variables and explanatory variables as in (16) from Section 2. Remember that $y_{t}$ is an $M \times 1$ vector containing data on $M$ dependent variables and $Z_{t}$ is an $M \times k$ matrix. In Section 2, we saw how $Z_{t}$ could be set up to either define an unrestricted or a restricted VAR. $Z_{t}$ can also contain exogenous explanatory variables. ${ }^{19}$ The basic TVP-VAR can be written as:

$$
y_{t}=Z_{t} \beta_{t}+\varepsilon_{t}
$$

and

$$
\beta_{t+1}=\beta_{t}+u_{t}
$$

where $\varepsilon_{t}$ is i.i.d. $N(0, \Sigma)$ and $u_{t}$ is i.i.d. $N(0, Q) \cdot \varepsilon_{t}$ and $u_{s}$ are independent of one another for all $s$ and $t$.

This model is similar to that used in Cogley and Sargent (2001). Bayesian inference in this model can be dealt with quite simply since it is a Normal linear state space model of the sort discussed in Section 3 of this monograph. Thus, the MCMC methods described in Section 3 can be used for Bayesian inference in the homoskedastic TVP-VAR. In many cases, this is all the researcher needs to know about TVP-VARs and Bayesian TVP-VARs work very well in practice. However, in some cases, this basic TVP-VAR can lead to poor results in practice. In the remainder of this section, we discuss how these poor results can arise and various extensions of the basic TVP-VAR which can help avoid them.

The poor results just referred to typically arise because the TVP-VAR has so many parameters to estimate. In Section 1 we saw how, even with the VAR, worries about the proliferation of parameters led to the use of priors such as the Minnesota prior or the SSVS prior. With so many parameters and relatively short macroeconomic time series, it can be hard to obtain precise estimates of coefficients. Thus, features of interest such as impulse responses can have very dispersed posterior distributions leading to wide credible intervals. Furthermore, the risk of over-fitting can be serious in some applications. In practice, it has been found that priors which exhibit shrinkage of various sorts can help mitigate these problems.

With the TVP-VAR, the proliferation of parameters problems is even more severe since it has $T$ times as many parameters to estimate. In Section 3, we saw how the state equation in a state space model can be interpreted as a hierarchical prior (see equation 30). And, in many applications, this prior provides enough shrinkage to yield reasonable results. Although it is worth noting that it is often a good idea to use a fairly tight prior for $Q$. For instance, if (33) is used

\footnotetext{
${ }^{19}$ The TVP-VAR where some of the coefficients are constant over time can be dealt with by adding $W_{t}$ as in (28) and (29).
} 
as a prior, then a careful choice of $\underline{\nu}_{Q}$ and $Q^{-1}$ can be important in producing sensible results. ${ }^{20}$ However, in some applications, it is desirable to introduce more prior information and we will describe several ways of doing so.

\subsubsection{Empirical Illustration of Bayesian Homoskedastic TVP-VAR Methods}

To illustrate Bayesian inference in the homoskedastic TVP-VAR, we use the same U.S. data set as before (see Section 2.3), which contains three variables: inflation, unemployment and interest rate and runs from 1953Q1 to 2006Q3. We set lag length to 2 .

We use a training sample prior of the type used by Primiceri (2005) where prior hyperparameters are set to OLS quantities calculating using a training sample of size $\tau$ (we use $\tau=40$ ). Thus, data through 1962Q4 is used to choose prior hyperparameter values and then estimation uses data beginning in 1963Q1. To be precise, the training sample prior uses $\beta_{O L S}$ which is the OLS estimate of the VAR coefficients in a constant-coefficient VAR and $V\left(\beta_{O L S}\right)$ which is its covariance matrix.

In this model, we need a prior for the initial state, $\beta_{0}$, the measurement equation error covariance matrix $\Sigma$ and the state equation error covariance matrix, $Q$. The first of these is:

$$
\beta_{0} \sim N\left(\beta_{O L S}, 4 \cdot V\left(\beta_{O L S}\right)\right),
$$

whereas the latter two are based on (32) with $\underline{\nu}=M+1, \underline{S}=I$ and (33) with $\underline{\nu}_{Q}=\tau, \underline{Q}=0.0001 \cdot \tau \cdot V\left(\beta_{O L S}\right)$.

Since the VAR regression coefficients are time-varying, there will be a different set of them in every time period. This typically will lead to far too many parameters to present in a table or graph. Here we will focus on impulse responses (defined as in Section 2.3). But even for these we have a different impulse response function at each point in time. ${ }^{21}$ Accordingly, Figure 4 plots impulse responses for three representative times: 1975Q1, 1981Q3 and 1996Q1. For the sake of brevity, we only present the impulse responses of each variable to a monetary policy shock (i.e. a shock in the interest rate equation).

Using the prior specified above, we see that there are differences in these responses in three different representative periods (1975Q1, 1981Q3 and 1996Q1). In this figure the posterior median is the solid line and the dotted lines are the $10^{t h}$ and $90^{t h}$ percentiles. Note in particular, the responses of inflation to the monetary policy shock. These are substantially different in our three different

\footnotetext{
${ }^{20}$ Attempts to use flat "noninformative" priors on $Q$ can go wrong since such flat priors actually can be quite informative, attaching large amounts of prior probability to large values of $Q$. Large values of $Q$ are associated with a high degree of variation on the VAR coefficients (i.e. much prior weight is attached to regions of the parameter space where the opposite of shrinkage occurs).

${ }^{21} \mathrm{It}$ is common practice to calculate the impulse responses at time $t$ using $\beta_{t}$ and simply ignoring the fact that it will change over time and we do so below. For more general treatments of Bayesian impulse response analysis see Koop (1996).
} 
representative periods and it is only in 1996Q1 that this impulse response looks similar to the comparable one in Figures 2 or 3 . The fact that we are finding such differences relative to the impulse responses reported in Section 2.3 indicates the potential importance of allowing for time variation in parameters.
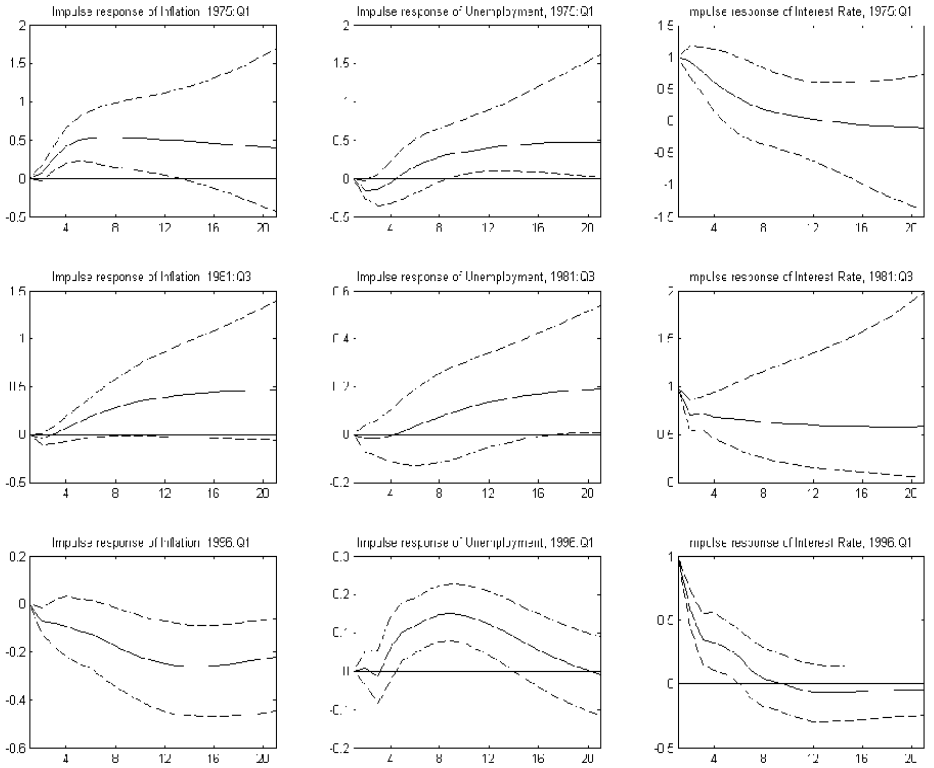

Figure 4: Posterior of impulse responses to monetary policy shock at different times

\subsubsection{Combining other Priors with the TVP Prior}

The Minnesota and SSVS Priors In Section 1, we described several priors that are commonly used with VARs and, when moving to TVP-VARs, an obvious thing to do is to try and combine them with the hierarchical prior defined by the state equation. This can be done in several ways. One approach, used in papers such as Ballabriga, Sebastian and Valles (1999), Canova and Ciccarelli (2004), and Canova (2007), involves combining the prior of the TVP-VAR with the Minnesota prior. This can be done by replacing (46) by

$$
\beta_{t+1}=A_{0} \beta_{t}+\left(I-A_{0}\right) \bar{\beta}_{0}+u_{t}
$$

where $A_{0}$ is a $k \times k$ matrix and $\bar{\beta}_{0}$ a $k \times 1$ vector. The matrices $A_{0}, \bar{\beta}_{0}$ and $Q$ can either be treated as unknown parameters or specific values of them can be chosen to reflect the Minnesota prior. For instance, Canova (2007, page 399) sets $\bar{\beta}_{0}$ and $Q$ to have forms based on the Minnesota prior and sets $A_{0}=c I$ 
where $c$ is a scalar. The reader is referred to Canova (2007) for precise details, but to provide a flavor of his recommendations, note that if $c=1$, then the traditional TVP-VAR prior implication that $E\left(\beta_{t+1}\right)=E\left(\beta_{t}\right)$ is obtained, but if $c=0$ then we have $E\left(\beta_{t+1}\right)=\bar{\beta}_{0}$. Canova (2007) recommends setting the elements of $\bar{\beta}_{0}$ corresponding to own lags to one and all other elements to zero, thus leading to the same prior mean as in the Minnesota prior. Canova (2007) sets $Q$ to values inspired by the prior covariance matrix of the Minnesota prior (similar to equation 8). The scalar $c$ can either be treated as an unknown parameter or a value can be selected for it (e.g. based on a training sample).

It is also possible to treat $A_{0}$ as a matrix of unknown parameters. To carry out Bayesian inference in such a model is usually straightforward since it requires only the addition of another block in an MCMC algorithm. In Section 3.2 we saw how an MCMC algorithm involving $p\left(\Sigma^{-1} \mid y^{T}, \beta^{T}\right), p\left(Q^{-1} \mid y^{T}, \beta^{T}\right)$ and $p\left(\beta^{T} \mid y^{T}, \Sigma, Q\right)$ could be used to carry out posterior simulation in the Normal linear state space model. If $A_{0}$ involves unknown parameters, then an MCMC algorithm involving $p\left(\Sigma^{-1} \mid y^{T}, \beta^{T}, A_{0}\right), p\left(Q^{-1} \mid y^{T}, \beta^{T}, A_{0}\right)$ and $p\left(\beta^{T} \mid y^{T}, \Sigma, Q, A_{0}\right)$ proceeds in exactly the same manner. Using the notation of Section 3.2, we can simply set $\Pi_{t}=A_{0}$ and the methods of that section can be directly used. To complete an MCMC algorithm requires draws from $p\left(A_{0} \mid y^{T}, \Sigma, Q, \beta^{T}\right)$. This will depend on the specification used for $A_{0}$, but typically this posterior conditional distribution is easy to derive using results for the VAR. That is, $p\left(A_{0} \mid y^{T}, \Sigma, Q, \beta^{T}\right)$ is a distribution which conditions on $\beta^{T}$, and (47) can be written in "VAR" form

$$
\beta_{t+1}-\bar{\beta}_{0}=A_{0}\left(\beta_{t}-\bar{\beta}_{0}\right)+u_{t},
$$

with "dependent variables" $\beta_{t+1}-\bar{\beta}_{0}$ and "lagged dependent variables" $\beta_{t}-\bar{\beta}_{0}$. In the context of the MCMC algorithm, these "dependent variables" and "lagged dependent variables" would be replaced with the drawn values.

Any prior can be used for $a_{0}=\operatorname{vec}\left(A_{0}\right)$ including any of the VAR priors described in Section 2. We will not provide exact formulae here, but note that they will be exactly as in Section 2 but with $y_{t}$ replaced by $\beta_{t+1}-\bar{\beta}_{0}$ and $x_{t}$ (or $Z_{t}$ ) replaced by $\beta_{t}-\bar{\beta}_{0}$.

One prior for $a_{0}$ of empirical interest is the SSVS prior of Section 2.2.3. It is interesting to consider what happens if we use the prior given in (22) and (23) for $a_{0}$ and set $\bar{\beta}_{0}=0 .{ }^{22}$ This implies that $a_{0 j}$ (the $j^{\text {th }}$ element of $a_{0}$ ) has a prior of the form:

$$
a_{0 j} \mid \gamma_{j} \sim\left(1-\gamma_{j}\right) N\left(0, \kappa_{0 j}^{2}\right)+\gamma_{j} N\left(0, \kappa_{1 j}^{2}\right),
$$

\footnotetext{
${ }^{22}$ If the variables in the VAR are in levels, then the researcher may wish to set the elements of $\bar{\beta}_{0}$ corresponding to own first lags to one, to reflect the common Minnesota prior belief in random walk behavior.
} 
where $\gamma_{j}$ is a dummy variable and $\kappa_{0 j}^{2}$ is very small (so that $a_{0 j}$ is constrained to be virtually zero), but $\kappa_{1 j}^{2}$ is large (so that $a_{0 j}$ is relatively unconstrained). The implication of combining the SSVS prior with the TVP prior is that we have a model which, with probability $\gamma_{j}$, says that $a_{0 j}$ is evolving according to a random walk in the usual TVP fashion, but with probability $\left(1-\gamma_{j}\right)$ is set to zero. Such a model is a useful one since it allows for change in VAR coefficients over time (which is potentially of great empirical importance), but also helps avoid over-parameterization problems by allowing for some lagged dependent variables to be deleted from the VAR. Another interesting approach with a similar methodology is given in Groen, Paap and Ravazzolo (2008).

Adding Another Layer to the Prior Hierarchy Another way to combine the TVP model with prior information from another source is by adding another state equation to the TVP-VAR (i.e. another layer in the prior hierarchy). The framework that follows is taken from Chib and Greenberg (1995) and has been used in macroeconomics by, among others, Ciccarelli and Rebucci (2002).

This involves writing the TVP-VAR as:

$$
\begin{aligned}
y_{t} & =Z_{t} \beta_{t}+\varepsilon_{t} \\
\beta_{t+1} & =A_{0} \theta_{t+1}+u_{t}, \\
\theta_{t+1} & =\theta_{t}+\eta_{t} .
\end{aligned}
$$

where all assumptions are as for the standard TVP-VAR, but we add the assumption that $\eta_{t}$ is i.i.d. $N(0, R)$ and $\eta_{t}$ is independent of the other errors in the model.

Note first that there is a sense in which this specification retains random walk evolution of the VAR coefficients since it can be written as:

$$
\begin{aligned}
y_{t} & =Z_{t} \beta_{t}+\varepsilon_{t} \\
\beta_{t+1} & =\beta_{t}+v_{t},
\end{aligned}
$$

where $v_{t}=A_{0} \eta_{t}+u_{t}-u_{t-1}$. In this sense, it is a TVP-VAR with random walk state equation but, unlike (46), the state equation errors have a particular MA(1) structure.

Another way of interpreting this model is by noting that, if $A_{0}$ is a square matrix, it expresses the conditional prior belief that

$$
E\left(\beta_{t+1} \mid \beta_{t}\right)=A_{0} \beta_{t}
$$

and, thus, is a combination of the random walk prior belief of the conventional TVP model with the prior beliefs contained in $A_{0} . A_{0}$ is typically treated as known.

Note that it is possible for $\theta_{t}$ to be of lower dimension than $\beta_{t}$ and this can be a useful way of making the model more parsimonious. For instance, Ciccarelli and Rebucci (2002) is a panel VAR application involving $G$ countries and, 
for each country, $k_{G}$ explanatory variables exist with time-varying coefficients. They specify

$$
A_{0}=\iota_{G} \otimes I_{k_{G}}
$$

which implies that there is a time-varying component in each coefficient which is common to all countries (rather than having $G$ different time-varying components arising from different components in each country). Thus, $\theta_{t}$ is $k_{G} \times 1$ whereas $\beta_{t} k_{G} G \times 1$.

Posterior computation in this model is described in Chib and Greenberg (1995). Alternatively, the posterior simulation methods for state space models described in Section 3 can be used, but with a more general specification for the state equation than that given there. Just as in the preceding section, if $A_{0}$ is to be treated as a matrix of unknown parameters any prior can be used and another block added to the MCMC algorithm. The form of this block will typically be simple since, conditional on the states, we have $\beta_{t+1}=A_{0} \theta_{t+1}+u_{t}$ which has the same structure as a multivariate regression model.

As one empirically-useful example, suppose we use the SSVS prior of Section 2.2.3 for $A_{0}$. Then we obtain a model where some VAR coefficients evolve according to random walks in the standard TVP fashion while others are (approximately) omitted from the model.

\subsubsection{Imposing Inequality Restrictions on the VAR Coefficients}

Empirical macroeconomists typically work with multivariate time series models that they believe to be non-explosive. Thus, in TVP-VAR models it can be desirable to impose stability on the TVP-VAR at each point in time. This has led papers such as Cogley and Sargent $(2001,2005)$ to restrict $\beta_{t}$ to satisfy the usual stability conditions for VARs for $t=1, . ., T$. This involves imposing the inequality restriction that the roots of the VAR polynomial defined by $\beta_{t}$ lie outside the unit circle. Indeed, in the absence of such a stability restriction (or a very tight prior), Bayesian TVP-VARs may place a large amount of a priori weight on explosive values for $\beta_{t}$ (e.g. the Minnesota prior is centered over a random walk which means it allocates prior weight to the explosive region of the parameter space). This can cause problems for empirical work. For instance, even a small amount of posterior probability in explosive regions for $\beta_{t}$ can lead to impulse responses or forecasts which have counter-intuitively large posterior means or standard deviations. Given that TVP-VARs have many parameters to estimate and the researcher often has relatively small data sets, the posterior standard deviation of $\beta_{t}$ can often be large. Thus, even if $\beta_{t}$ truly is stable and its posterior mean indicates stability, it is not unusual for large posterior variances to imply that appreciable posterior probability is allocated to the explosive region.

The preceding paragraph motivates one case where the researcher might wish to impose inequality restrictions on $\beta_{t}$ in order to surmount potential overparameterization problems which might arise in the TVP-VAR. Other inequality 
restrictions are also possible. In theory, imposing inequality restrictions is a good way of reducing over-parameterization problems. In practice, there is one problem with this strategy. This problem is that standard state space methods for the Normal linear state space models (see Section 3) cannot be used without some modification. Remember that MCMC methods for this model involved taking draws from $p\left(\beta^{T} \mid y^{T}, \Sigma, Q\right)$ and that there are many efficient algorithms for doing so (e.g. Carter and Kohn, 1994, Fruhwirth-Schnatter, 1994, DeJong and Shephard, 1995 and Durbin and Koopman, 2002). However, all of these algorithms are derived using properties of the multivariate Normal distribution which do not carry over to the multivariate Normal distribution subject to inequality constraints.

Two methods have been proposed to impose stability restrictions (or other inequality restrictions) on TVP-VARs. These are discussed and compared in Koop and Potter (2009). The first of these involves using a standard algorithm such as that of Carter and Kohn (1994) for drawing $\beta^{T}$ in the unrestricted VAR. If any drawn $\beta_{t}$ violates the inequality restriction then the entire vector $\beta^{T}$ is rejected. ${ }^{23}$ If every element of $\beta^{T}$ satisfies the inequality restriction then $\beta^{T}$ is accepted with a certain probability (the formula for this probability is given in Koop and Potter, 2009). A potential problem with this algorithm is that it is possible for it to get stuck, rejecting virtually every $\beta^{T}$. In theory, if enough draws are taken this MCMC algorithm can be highly accurate, in practice "enough draws" can be so many that the algorithm simply cannot produce accurate results in a feasible amount of computer time.

In the case where no inequality restrictions are imposed, the advantage of algorithms such as that of Carter and Kohn (1994) is that they are multi-move algorithms. This means that they provide a draw for the entire vector $\beta^{T}$ from $p\left(\beta^{T} \mid y^{T}, \Sigma, Q\right)$ directly. The logic of MCMC suggests that it would also be valid to draw $\beta_{t}$ for $t=1, . ., T$ one at a time from $p\left(\beta_{t} \mid y^{T}, \Sigma, Q, \beta_{-t}\right)$ where $\beta_{-t}=\left(\beta_{1}^{\prime}, . ., \beta_{t-1}, \beta_{t+1}, . ., \beta_{T}^{\prime}\right)^{\prime}$. It is indeed the case that this is also valid thing to do. However, it is rarely done in practice since such single-move algorithms will be slow to mix. That is, they will tend to produce a highly correlated series of draws which means that, relative to multi-move algorithms, more draws must be taken to achieve a desired level of accuracy. The second algorithm proposed for the TVP-VAR subject to inequality restrictions is a single-move algorithm. This algorithm does have the disadvantage just noted - that it is slow mixing. But it is possible that this disadvantage is outweighed by the advantage that the single-move algorithm does not run into the problem noted above for the multi-move algorithm (i.e. that the multi-move algorithm can get stuck and reject every draw).

Koop and Potter (2009) provide full details of both these algorithms and weigh their advantages and disadvantages in a macroeconomic application.

\footnotetext{
${ }^{23}$ It can be shown that the strategy of rejecting only individual $\beta_{t}$ which violate the inequality restriction is not a valid one.
} 


\subsubsection{Dynamic Mixture Models}

Another way of tightening the parameterization of the TVP-VAR is through the dynamic mixture model approach of Gerlach, Carter and Kohn (2000). This has recently been used in models of relevance for empirical macroeconomics in Giordani, Kohn and van Dijk (2007) and Giordani and Kohn (2008) and applied to TVP-VARs in Koop, Leon-Gonzalez and Strachan (2009).

To explain the usefulness of dynamic mixture modelling for TVP-VAR analysis, return to the general form for the Normal linear state space model given in (28) and (29) and remember that this model depended on so-called system matrices, $Z_{t}, Q_{t}, \Pi_{t}, W_{t}$ and $\Sigma_{t}$. Dynamic mixture models allow for any or all of these system matrices to depend on an $s \times 1$ vector $\widetilde{K}_{t}$. Gerlach, Carter and Kohn (2000) discuss how this specification results in a mixtures of Normals representation for $y_{t}$ and, hence, the terminology dynamic mixture (or mixture innovation) model arises. The contribution of Gerlach, Carter and Kohn (2000) is to develop an efficient algorithm for posterior simulation for this class of models. The efficiency gains occur since the states are integrated out and $\widetilde{K}=\left(\widetilde{K}_{1}, . ., \widetilde{K}_{T}\right)^{\prime}$ is drawn unconditionally (i.e. not conditional on the states). A simple alternative algorithm would involve drawing from the posterior for $\widetilde{K}$ conditional on $\beta^{T}$ (and the posterior for $\beta^{T}$ conditional on $\widetilde{K}$ ). Such a strategy can be shown to produce a chain of draws which is very slow to mix. The Gerlach, Carter and Kohn (2000) algorithm requires only that $\widetilde{K}_{t}$ be Markov (i.e. $\left.p\left(\widetilde{K}_{t} \mid \widetilde{K}_{t-1}, . ., \widetilde{K}_{1}\right)=p\left(\widetilde{K}_{t} \mid \widetilde{K}_{t-1}\right)\right)$ and is particularly simple if $\widetilde{K}_{t}$ is a discrete random variable. We will not provide details of their algorithm here, but refer the reader to Gerlach, Carter and Kohn (2000) or Giordani and Kohn (2008). This algorithm is available on the Matlab website associated with this monograph.

The dynamic mixture framework can be used in many ways in empirical macroeconomics, here we illustrate one useful way. Consider the following TVPVAR:

$$
y_{t}=Z_{t} \beta_{t}+\varepsilon_{t}
$$

and

$$
\beta_{t+1}=\beta_{t}+u_{t},
$$

where $\varepsilon_{t}$ is i.i.d. $N(0, \Sigma)$ and $u_{t}$ is i.i.d. $N\left(0, \widetilde{K}_{t} Q\right)$. This model is exactly the same as the TVP-VAR of Section 4.1, except for the error covariance matrix in the state equation. Let $\widetilde{K}_{t} \in\{0,1\}$ and assume a hierarchical prior for it of the following form:

$$
\begin{aligned}
& p\left(\widetilde{K}_{t}=1\right)=q . \\
& p\left(\widetilde{K}_{t}=0\right)=1-q
\end{aligned}
$$


where $q$ is an unknown parameter. ${ }^{24}$ that:

This is a simple example of a dynamic mixture model. It has the property

$$
\begin{aligned}
& \beta_{t+1}=\beta_{t}+u_{t} \text { if } \widetilde{K}_{t}=1 \\
& \beta_{t+1}=\beta_{t} \text { if } \widetilde{K}_{t}=0
\end{aligned} .
$$

In words, the VAR coefficients can change at time $t$ (if $\widetilde{K}_{t}=1$ ), but can also remain constant (if $\widetilde{K}_{t}=0$ ) and $q$ is the probability that the coefficients change. $\widetilde{K}_{t}$ and $q$ are estimated in a data-based fashion. Thus, this model can have the full flexibility of the TVP-VAR if the data warrant it (in the sense that it can select $\widetilde{K}_{t}=1$ for $\left.t=1, . ., T\right)$. But it can also select a much more parsimonious representation. In the extreme, if $\widetilde{K}_{t}=0$ for $t=1, . ., T$ then we have the VAR without time-varying parameters.

This simple example allows either for all the VAR coefficients to change at time $t$ (if $\widetilde{K}_{t}=1$ ) or none (if $\widetilde{K}_{t}=0$ ). More sophisticated models can allow for some parameters to change but not others. For instance, $\widetilde{K}_{t}$ could be a vector of $M$ elements, each being applied to one of the equations in the VAR. Such a model would allow the VAR coefficients in some equations to change, but remain constant in other equations. In models with multivariate stochastic volatility, $\widetilde{K}_{t}$ could contain elements which control the variation in the measurement error covariance matrix, $\Sigma_{t}$. This avenue is pursued in Koop, Leon-Gonzalez and Strachan (2009). Many other possibilities exist (see, e.g., the time-varying dimension models of Chan, Koop, Leon-Gonzalez and Strachan, 2009) and the advantage of the dynamic mixture framework is that there exists a well-developed, well-understood set of MCMC algorithms that make Bayesian inference straightforward.

\subsection{TVP-VARs with Stochastic Volatility}

Thus far, we have focussed on the homoskedastic TVP-VAR, assuming the error covariance matrix, $\Sigma$ to be constant. However, we have argued above (see Section 3.3) that volatility issues are often very important in empirical macroeconomics. Thus, in most cases, it is important to allow for multivariate stochastic volatility in the TVP-VAR. Section 3.3.2 discusses multivariate stochastic volatility, noting that there are many possible specifications for $\Sigma_{t}$. Particular approaches used in Cogley and Sargent (2005) and Primiceri (2005) are described in detail. All that we have to note here is that either of these approaches (or any other alternative) can be added to the homoskedastic TVP-VAR.

With regards to Bayesian inference using MCMC methods, we need only add another block to our algorithm to draw $\Sigma_{t}$ for $t=1, . ., T$. That is, with the homoskedastic TVP-VAR we saw how an MCMC algorithm involving $p\left(Q^{-1} \mid y^{T}, \beta^{T}\right), p\left(\beta^{T} \mid y^{T}, \Sigma, Q\right)$ and $p\left(\Sigma^{-1} \mid y^{T}, \beta^{T}\right)$ could be used. When

\footnotetext{
${ }^{24}$ This specification for $\widetilde{K}_{t}$ is Markov in the degenerate sense that an independent process is a special case of Markov process.
} 
adding multivariate stochastic volatility, the first of these densities is unchanged. The second, $p\left(\beta^{T} \mid y^{T}, \Sigma, Q\right)$, becomes $p\left(\beta^{T} \mid y^{T}, \Sigma_{1}, . ., \Sigma_{T}, Q\right)$ which can be drawn from using any of the algorithms (e.g. Carter and Kohn, 1994) for the Normal linear state space model mentioned previously. Finally, $p\left(\Sigma^{-1} \mid y^{T}, \beta^{T}\right)$ is replaced by $p\left(\Sigma_{1}^{-1}, . ., \Sigma_{T}^{-1} \mid y^{T}, \beta^{T}\right)$. Draws from this posterior conditional can be taken as described in Section 3.3.2.

\subsection{Empirical Illustration of Bayesian Inference in TVP- VARs with Stochastic Volatility}

We continue our empirical illustration of Section 4.1.1 which involved a homoskedastic TVP-VAR. All details of the empirical are as in Section 4.1.1 except we additionally allow for multivariate stochastic volatility as in Primiceri (2005). The prior for the parameters relating to the multivariate stochastic volatility are specified as in Primiceri (2005).

For the sake of brevity, we do not present impulse responses (these are similar to those presented in Primiceri, 2005). Instead we present information relating to the multivariate stochastic volatility. Figure 6 presents the time-varying standard deviations of the errors in the three equations of our TVP-VAR (i.e. the posterior means of the square roots of the diagonal element of $\Sigma_{t}$ ). Figure 5 shows that there is substantial time variation in the error variances in all equations. In particular, it can be seen that 1970s was a very volatile period for the US economy, while the monetarist experiment of the early 1980s is also with instability. However, after the early 1980s volatility is greatly reduced in all equations. This latter period has come to be known as the Great Moderation of the business cycle. 


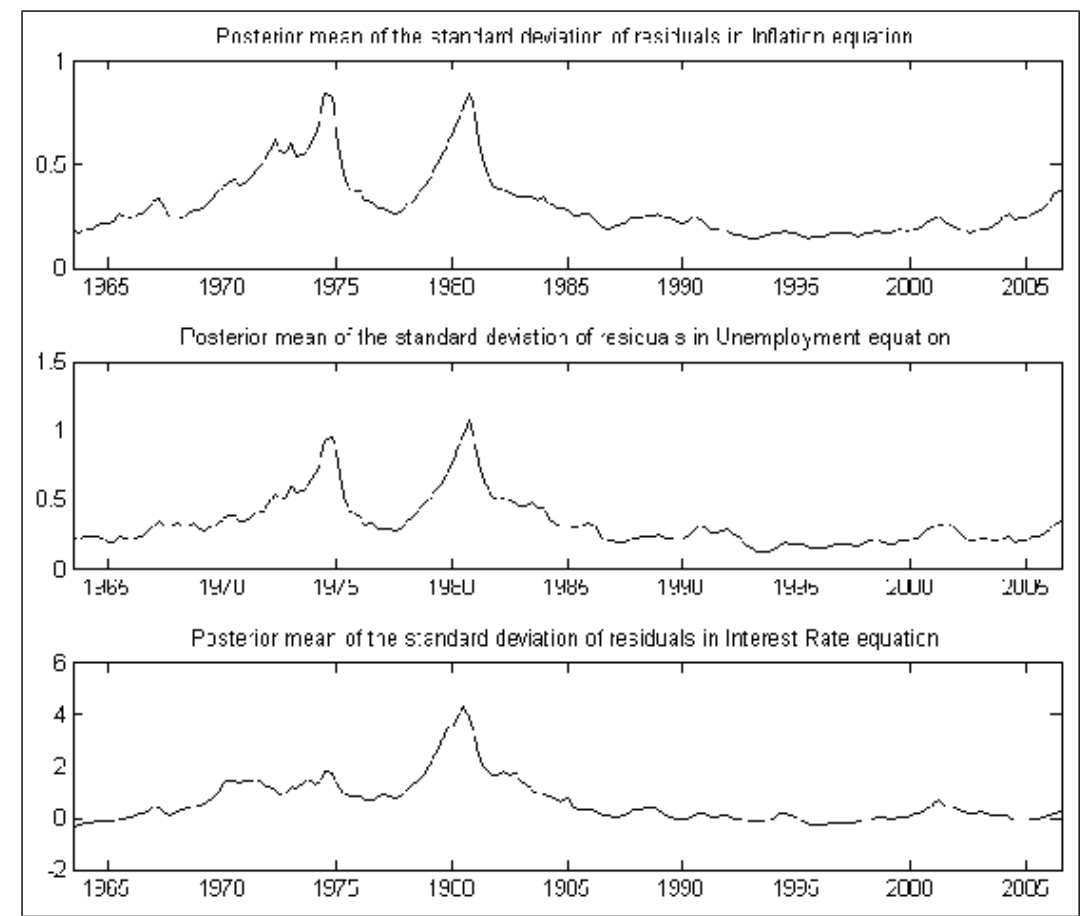

Figure 5: Time-varying volatilities of errors in the three equations of the TVP-VAR

\section{Factor Methods}

The VAR and TVP-VAR methods we have discussed so far are typically used when the number of variables in the model is relatively small (e.g. three or four and rarely more than ten). ${ }^{25}$ However, in most modern economies, the macroeconomist will potentially have dozens or hundreds of time series variables to work with. Especially when forecasting, the researcher wants to include as much information as possible, and it can be desirable to work with as many variables as possible. This can lead to models with a large number of variables and a proliferation of parameters. Accordingly, researchers have sought ways of extracting the information in data sets with many variables while, at the same time, keeping the model parsimonious. Beginning with Geweke (1977) factor models have been the most common way of achieving this goal (see Stock and Watson 2006 for a recent survey). Applications such as Forni and Reichlin (1998), Stock and Watson (1999, 2002), Bernanke and Boivin (2003), have popularized factor methods among macroeconomists and Geweke and Zhou (1996), Otrok

\footnotetext{
${ }^{25}$ An important exception is Banbura, Giannone and Reichlin (2008) which uses Bayesian VARs (with time-invariant coefficients) with up to 130 variables
} 
and Whiteman (1998) and Kose, Otrok and Whiteman (2003), among many others, stimulated the interest of Bayesians. Papers such as Bernanke, Boivin and Eliasz (2005) and Stock and Watson (2005) have combined factor methods with VAR methods. More recently, papers such as Del Negro and Otrok (2008) and Korobilis (2009a) provide further TVP extensions to these models. In this section, we will describe dynamic factor models and their extensions to factor augmented VARs (FAVARs) and TVP-FAVARs. As we shall see, these models can be interpreted as state space models and Bayesian MCMC algorithms that use our previously-discussed algorithms for state space models can be used.

\subsection{Introduction}

We will retain our notation where $y_{t}$ is an $M \times 1$ vector of time series variables, but now $M$ will be very large and let $y_{i t}$ denote a particular variable. A simple static factor model is (see Lopes and West, 2004):

$$
y_{t}=\lambda_{0}+\lambda f_{t}+\varepsilon_{t}
$$

The key aspect of the factor model is the introduction of $f_{t}$ which is a $q \times 1$ vector of unobserved latent factors (where $q<<M$ ) which contains information extracted from all the $M$ variables. The factors are common to every dependent variable (i.e. the same $f_{t}$ occurs in every equation for $y_{i t}$ for $i=1, \ldots, M$ ), but they may have different coefficients ( $\lambda$ which is a $M \times q$ matrix of so-called factor loadings). Also, the equation for every dependent variable has its own intercept (i.e. $\lambda_{0}$ is $M \times 1$ vector of parameters). $\varepsilon_{t}$ is i.i.d. $N(0, \Sigma)$ although, for reasons explained below, typically $\Sigma$ is restricted to be a diagonal matrix. Different factor models arise from the assumptions about the factors. For example, the simplest case would be to assume that the factors come from a standard Normal density, $f_{t} \sim N(0, I)$. This implies that the covariance matrix of the observed data can be written as:

$$
\operatorname{var}(y)=\lambda \lambda^{\prime}+\Sigma .
$$

Alternatively, if we assume that the factors have a (not necessarily diagonal) covariance matrix $\Sigma^{f}$, the decomposition becomes

$$
\operatorname{var}(y)=\lambda \Sigma^{f} \lambda^{\prime}+\Sigma .
$$

Even in this simple static framework, many extensions are possible. For example, Pitt and Shephard (1999) assume a factor stochastic volatility specification (i.e. a diagonal factor covariance matrix $\Sigma_{t}^{f}$ which varies over time, with diagonal elements following a geometric random walk). West (2003) uses SSVS on the parameters $\left(\lambda_{0}, \lambda\right)$.

We write out these covariance matrices to illustrate the identification issues which arise in factor models. In general, $\operatorname{var}(y)$ will have $\frac{M(M+1)}{2}$ elements

which can be estimated. However, without further restrictions, $\Sigma$ and $\lambda$ (or $\Sigma, \Sigma_{f}$ and $\lambda$ ) will have many more elements than this. The popular restriction that $\Sigma$ is a diagonal matrix implies that all the commonalities across variables 
occur through the factors and that the individual elements of $\varepsilon_{t}$ are purely shocks which are idiosyncratic to each variable. But additional identifying restrictions are typically required. Lopes and West (2004) and Geweke and Zhou (1996) give a clear explanation of why identification is not achieved in the simple factor model. Below we will discuss more flexible factor models, but we stress that this added flexibility requires the imposition of more identification restrictions. There is not a single, universally agreed-upon method for achieving identification in these models. Below, we make particular choices that have been used in the literature, but note that others are possible.

\subsection{The Dynamic Factor Model}

In macroeconomics, it is common to extend the static factor model to allow for the dynamic properties which characterize macroeconomic variables. This leads us to dynamic factor models (DFMs). A popular DFM is:

$$
\begin{aligned}
& y_{i t}=\lambda_{0 i}+\lambda_{i} f_{t}+\varepsilon_{i t} \\
& f_{t}=\Phi_{1} f_{t-1}+. .+\Phi_{p} f_{t-p}+\varepsilon_{t}^{f} \\
& \varepsilon_{i t}=\phi_{i 1} \varepsilon_{i t-1}+. .+\phi_{i p_{i}} \varepsilon_{i t-p_{i}}+u_{i t}
\end{aligned}
$$

where $f_{t}$ is defined as in (49), $\lambda_{i}$ which is a $1 \times q$ vector of factor loadings. Also, the equation for every dependent variable has its own intercept, $\lambda_{0 i}$. The error in each equation, $\varepsilon_{i t}$, may be autocorrelated, as specified in the third equation in (50) which assumes $u_{i t}$ to be i.i.d. $N\left(0, \sigma_{i}^{2}\right)$. The vector of factors is assumed to follow a VAR process with $\varepsilon_{t}^{f}$ being i.i.d. $N\left(0, \Sigma^{f}\right)$. The errors, $u_{i t}$ are independent over $i, t$ and of $\varepsilon_{t}^{f}$.

Many slight modifications of the DFM given in (50) have been used in the literature. But this specification is a popular one so we will discuss factor models using this framework. Note that it incorporates many identifying assumptions and other assumptions to ensure parsimony. In particular, the assumption that $\Sigma^{f}=I$ is a common identifying assumption. ${ }^{26}$ If $\Sigma^{f}$ is left unrestricted, then it is impossible to separately identify $\lambda_{i}$ and $\Sigma^{f}$. Similarly, the identifying assumption that $u_{i t}$ is uncorrelated with $u_{j t}$ (for $i \neq j$ ) and $\varepsilon_{t}^{f}$ is a standard one which assures that the co-movements in the different variables in $y_{t}$ arises from the factors. Even with these assumptions, there is a lack of identification since the first equation of (50) can be replaced by:

$$
y_{i t}=\lambda_{0 i}+\lambda_{i} C^{\prime} C f_{t}+\varepsilon_{i t},
$$

where $C$ is any orthonormal matrix. ${ }^{27}$ Thus, (50) is observationally equivalent to a model with factors $C f_{t}$ and factor loadings $\lambda_{i} C^{\prime}$. One way of surmounting this issue is to impose restrictions on $\lambda_{i}$ (as is done below in our empirical example). A further discussion of various DFMs and identification is provided,

\footnotetext{
${ }^{26}$ However, if (as below and in Pitt and Shephard, 1999) the researcher wishes to allow for factor stochastic volatility then this identifying assumption cannot be made and an alternative one is necessary.

${ }^{27}$ An orthonormal matrix has the property that $C^{\prime} C=I$.
} 
e.g., in Stock and Watson (2005). See also Sentana and Fiorentini (2001) for a deeper discussion of identification issues.

To simplify the following discussion, we will assume the errors in the measurement equation are not autocorrelated (i.e. $\varepsilon_{i t}$ are i.i.d. $N\left(0, \sigma_{i}^{2}\right)$ and, thus, $\left.\phi_{i 1}=. .=\phi_{i p_{i}}=0\right)$. We do this not because the extension to autocorrelated errors is empirically unimportant (it may be important), but because it involves a straightforward addition of other blocks to the MCMC algorithm of a standard form. That is, adding AR (or ARMA) errors to a regression model such as the first equation of (50) involves standard methods (see, e.g., Chib and Greenberg, 1994). To be precise, the Chib and Greenberg (1994) algorithm will produce draws of $\phi_{i 1}, . ., \phi_{i p_{i}}$ and these can be plugged into the usual quasi-differencing operator for AR models and this operator can be applied to the first equation in (50). The methods described below can then be used to draw all the other parameters of this model, except that $y_{i t}$ and $f_{t}$ will be replaced by quasi-differenced versions.

\subsubsection{Replacing Factors by Estimates: Principal Components}

Before discussing a full Bayesian analysis of the DFM which (correctly) treats $f_{t}$ as a vector of unobserved latent variables, it is worth noting a simple approximation that may be convenient in practice. This approximation is used, e.g., in Koop and Potter (2004). It involves noting that the DFM has approximately the same structure as the regression model:

$$
y_{i t}=\lambda_{0 i}+\widetilde{\lambda}_{0 i} f_{t}+. .+\widetilde{\lambda}_{p i} f_{t-p}+\widetilde{\varepsilon}_{i t} .
$$

Thus, if $f_{t}$ were known we could use Bayesian methods for the multivariate Normal regression model to estimate or forecast with the DFM.

However, it is common to use principal components methods to approximate $f_{t} .{ }^{28}$ Hence, approximate methods for Bayesian analysis of the DFM can be carried out by simply replacing $f_{t}(51)$ by a principal components estimate and using regression methods.

\subsubsection{Treating Factors as Unobserved Latent Variables}

It is also not difficult to treat the factors as unobserved latent variables using Bayesian methods for state space models that were discussed in Section 3. This is particularly easy to see if we ignore the AR structure of the errors in the measurement equation and write the DFM as

$$
\begin{aligned}
& y_{i t}=\lambda_{0 i}+\lambda_{i} f_{t}+\varepsilon_{i t} \\
& f_{t}=\Phi_{1} f_{t-1}+. .+\Phi_{p} f_{t-p}+\varepsilon_{t}^{f}
\end{aligned}
$$

for $i=1, . ., M$ where $\varepsilon_{i t}$ is i.i.d. $N\left(0, \sigma_{i}^{2}\right)$ and $\varepsilon_{t}^{f}$ is i.i.d. $N\left(0, \Sigma^{f}\right)$. In this form it can be seen clearly that the DFM is a Normal linear state space model of

\footnotetext{
${ }^{28}$ If $Y$ is the $T \times M$ matrix containing all the variables and $W$ is the $M \times q$ matrix containing the eigenvectors corresponding to the $q$ largest eigenvalues of $Y^{\prime} Y$, then $F=Y W$ produces an estimate of the matrix of the factors.
} 
the form given in (28) and (29). Thus all the methods for posterior simulation introduced in Section 3 can be used to carry out Bayesian inference. In the following we provide some additional detail about the steps involved.

Note first that, conditional on the model's parameters, $\Sigma^{f}, \Phi_{1}, . ., \Phi_{p}, \lambda_{0 i}, \lambda_{i}, \sigma_{i}^{2}$ for $i=1, . ., M$, any of the standard algorithms for state space models such as that of Carter and Kohn (1994) can be used to draw the factors. But conditional on the factors, the measurement equations are just $M$ Normal linear regression models. Next note that the assumption that $\varepsilon_{i t}$ is independent of $\varepsilon_{j t}$ for $i \neq j$ means that the posteriors for $\lambda_{0 i}, \lambda_{i}, \sigma_{i}^{2}$ in the $M$ equations are independent over $i$ and, hence, the parameters for each equation can be drawn one at a time. Finally, conditional on the factors, the state equation becomes a VAR and the methods for Bayesian analysis in VARs of Section 2 can be used. Thus, every block in the resulting Gibbs sampler takes a standard form (e.g. no Metropolis-Hastings steps are required). Details on the derivations for this or related models can be found in many places, including Geweke and Zhou (1996), Kim and Nelson (1999), Lopes and West (2004) or Del Negro and Schorfheide (2010). Lopes and West (2004) discusses the choice of $q$, the number of factors.

The preceding paragraph describes a correct MCMC algorithm that involves blocks taken from MCMC algorithms described previously in this monograph. However, the fact that this algorithm draws the model's parameters conditional on the factors has been found by Chib, Nardari, and Shephard (2006) and Chan and Jeliazkov (2009) to lead to poor MCMC performance. That is, the sequence of draws produced by the algorithm can be highly autocorrelated, meaning that a large number of draws can be required to achieve accurate estimates. These papers provide alternative algorithms which integrate out the factors and, thus, do not run into this problem.

\subsubsection{Impulse Response Analysis in the DFM}

In our discussion of impulse response analysis in VAR models, we emphasized how it is typically done based on a structural VAR:

$$
C_{0} y_{t}=c_{0}+\sum_{j=1}^{p} C_{j} y_{t-j}+u_{t}
$$

or $C(L) y_{t}=c_{0}+u_{t}$ where $u_{t}$ is i.i.d. $N(0, I), C(L)=C_{0}-\sum_{j=1}^{p} C_{j} L^{p}$ and $L$ is the lag operator. In fact, impulse responses are coefficients in the vector moving averaging (VMA) representation:

$$
y_{t}=\widetilde{c}_{0}+C(L)^{-1} u_{t},
$$

where $\widetilde{c}_{0}$ is a vector of intercepts $\left(\widetilde{c}_{0}=C(L)^{-1} c_{0}\right)$. To be precise, the response of the $i^{\text {th }}$ variable to the $j^{\text {th }}$ structural error $h$ periods in the future, will be the $(i j)^{t h}$ element of the VMA coefficient matrix on $u_{t-h}$. This makes clear that what is required for impulse response analysis is a VMA representation and a method for structurally identifying shocks through a choice of $C_{0}$. 
With the DFM, we can obtain the VMA representation for $y_{t}$. By substituting the state equation in (52) into the measurement equation we can obtain:

$$
\begin{aligned}
y_{t} & =\varepsilon_{t}+\lambda \Phi(L)^{-1} \varepsilon_{t}^{f} \\
& =B(L) \eta_{t}
\end{aligned}
$$

where $\Phi(L)=I-\Phi_{1} L-. .-\Phi_{p} L^{p}$ and, for notational simplicity, we have suppressed the intercept and are still assuming $\varepsilon_{t}$ to be serially uncorrelated. Adding such extensions is conceptually straightforward (e.g. implying $\varepsilon_{t-1}, . ., \varepsilon_{t-p}$ would be included in the VMA representation).

In the VMA form it can be seen that standard approaches to impulse response analysis run into trouble when the DFM is used since the errors in the VMA are a combination of the measurement equation errors, $\varepsilon_{t}$, and the state equation errors, $\varepsilon_{t}^{f}$. For instance, in the VAR it is common to identify the structural shocks by assuming $C_{0}$ to be a lower-triangular matrix. If the interest rate were the last element of $y_{t}$ this would ensure that the error in the interest rate equation had no immediate effect on the other variables thus identifying it as a monetary policy shock under control of the central bank (i.e. the shock will be proportional to the change in interest rates). With the DFM, a monetary policy shock defined by assuming $C_{0}$ to be lower triangular will not purely reflect the interest rate change, but will reflect the change in the interest rate and relevant element of $\varepsilon_{t}^{f}$. Thus, impulse response analysis in DFMs is problematic: it is difficult to identify an economically-sensible structural shock to measure impulse responses to. This motivates the use of FAVARs. In one sense, these are simply a different form for writing the DFM but implicitly involve a restriction which allows for economically-sensible impulse response analysis. ${ }^{29}$

\subsection{The Factor Augmented VAR (FAVAR)}

DFMs are commonly used for forecasting. However, interest in combining the theoretical insights provided by VARs with factor methods' ability to extract information in large data sets motivates the development of factor augmented VARs or FAVARs. For instance, a VAR with an identifying assumption which isolates a monetary policy shock can be used to calculate impulse responses which measure the effect of monetary policy. As we have seen, such theoretical insights are hard to obtain in the DFM. However, VARs typically involve only a few variables and it is possible that this means important economic information is excluded. ${ }^{30}$ This suggests that combining factor methods, which extract the information in hundreds of variables, with VAR methods might be productive.

\footnotetext{
${ }^{29}$ This is the interpretation of the FAVAR given by Stock and Watson (2005) who begin by writing the FAVAR as simply being a DFM written in VAR form.

${ }^{30}$ As an example, VARs with a small number of variables sometimes lead to counter-intuitive impulse responses such as the commonly noted price puzzle (e.g. where increases in interest rates seem to increase inflation). Such puzzles often vanish when more variables are included in the VAR suggesting that VARs with small numbers of variables may be mis-specified.
} 
This is done in papers such as Bernanke, Boivin and Eliasz (2005) and Belviso and Milani (2006).

The FAVAR modifies a DFM such as (52) by adding other explanatory variables to the $M$ measurement equations:

$$
y_{i t}=\lambda_{0 i}+\lambda_{i} f_{t}+\gamma_{i} r_{t}+\varepsilon_{i t}
$$

where $r_{t}$ is a $k_{r} \times 1$ vector of observed variables. For instance, Bernanke, Boivin and Eliasz (2005) set $r_{t}$ to be the Fed Funds rate (a monetary policy instrument) and, thus, $k_{r}=1$. All other assumptions about the measurement equation are the same as for the DFM.

The FAVAR extends the state equation for the factors to also allow for $r_{t}$ to have a VAR form. In particular, the state equation becomes:

$$
\left(\begin{array}{c}
f_{t} \\
r_{t}
\end{array}\right)=\widetilde{\Phi}_{1}\left(\begin{array}{c}
f_{t-1} \\
r_{t-1}
\end{array}\right)+. .+\widetilde{\Phi}_{p}\left(\begin{array}{c}
f_{t-p} \\
r_{t-p}
\end{array}\right)+\widetilde{\varepsilon}_{t}^{f}
$$

where all state equation assumptions are the same as for the DFM with the extension that $\widetilde{\varepsilon}_{t}^{f}$ is i.i.d. $N\left(0, \widetilde{\Sigma}^{f}\right)$.

We will not describe the MCMC algorithm for carrying out Bayesian inference in the FAVAR since it is very similar to that for the DFM. ${ }^{31}$ That is, (53) and (54) is a Normal linear state space model and, thus, standard methods (e.g. from Carter and Kohn, 1994) described in Section 3 can be used to draw the latent factors (conditional on all other model parameters). Conditional on the factors, the measurement equations are simply univariate Normal linear regression models for which Bayesian inference is standard. Finally, conditional on the factors, (54) is a VAR for which Bayesian methods have been discussed in this monograph.

\subsubsection{Impulse Response Analysis in the FAVAR}

With the FAVAR, impulse responses of all the variables in $y_{t}$ to the shocks associated with $r_{t}$ can be calculated using standard methods. For instance, if $r_{t}$ is the interest rate and, thus, the error in its equation is the monetary policy shock, then the response of any of the variables in $y_{t}$ to the monetary policy shock can be calculated using similar methods as for the VAR. To see this note that the FAVAR model can be written as:

$$
\begin{aligned}
& \left(\begin{array}{l}
y_{t} \\
r_{t}
\end{array}\right)=\left[\begin{array}{ll}
\lambda & \gamma \\
0 & 1
\end{array}\right]\left(\begin{array}{l}
f_{t} \\
r_{t}
\end{array}\right)+\widetilde{\varepsilon}_{t} \\
& \left(\begin{array}{l}
f_{t} \\
r_{t}
\end{array}\right)=\widetilde{\Phi}_{1}\left(\begin{array}{l}
f_{t-1} \\
r_{t-1}
\end{array}\right)+. .+\widetilde{\Phi}_{p}\left(\begin{array}{l}
f_{t-p} \\
r_{t-p}
\end{array}\right)+\widetilde{\varepsilon}_{t}^{f}
\end{aligned}
$$

\footnotetext{
${ }^{31}$ The working paper version of Bernanke, Boivin and Eliasz (2005) has an appendix which provides complete details. See also the Matlab manual on the website associated with this monograph.
} 
where $\widetilde{\varepsilon}_{t}=\left(\varepsilon_{t}^{\prime}, 0\right)^{\prime}$ and $\gamma$ is an $M \times k_{r}$ matrix containing the $\gamma_{i} \mathrm{~s}$. As for the DFM model, for notational simplicity, we have suppressed the intercept and assumed $\varepsilon_{t}$ to be serially uncorrelated. Adding such extensions is straightforward.

If we write the second equation in VMA form as $\left(\begin{array}{c}f_{t} \\ r_{t}\end{array}\right)=\widetilde{\Phi}(L)^{-1} \widetilde{\varepsilon}_{t}^{f}$ (where $\widetilde{\Phi}(L)=I-\widetilde{\Phi}_{1} L-. .-\widetilde{\Phi} L^{p}$ ) and substitute into the first equation, we obtain:

$$
\begin{aligned}
\left(\begin{array}{c}
y_{t} \\
r_{t}
\end{array}\right) & =\left[\begin{array}{cc}
\lambda & \gamma \\
0 & 1
\end{array}\right] \widetilde{\Phi}(L)^{-1} \widetilde{\varepsilon}_{t}^{f}+\widetilde{\varepsilon}_{t} \\
& =\widetilde{B}(L) \eta_{t} .
\end{aligned}
$$

Thus, we have a VMA form which can be used for impulse response analysis. But consider the last $k_{r}$ elements of $\eta_{t}$ which will be associated with the equations for $r_{t}$. Unlike with the DFM, these VMA errors are purely the errors associated with the VAR for $r_{t}$. This can be seen by noting that the last $k_{r}$ elements of $\widetilde{\varepsilon}_{t}$ are zero and thus the corresponding elements of $\eta_{t}$ will only reflect corresponding elements of $\widetilde{\varepsilon}_{t}^{f}$ which are errors in equations having $r_{t}$ as dependent variables. Unlike in the DFM, they do not combine state equation errors with measurement equation errors. For instance, if $r_{t}$ is an interest rate and structural identification is achieved by assuming $C_{0}$ (see equation 14) to be lower-triangular, then the structural shock to the interest rate equation is truly proportional to a change in the interest rate and the response to such a monetary policy shock has an economically-sensible interpretation.

Remember that, as with any factor model, we require identification restrictions (e.g. principal components methods implicitly involve an identification restriction that the factors are orthogonal, but other restrictions are possible). In order to do structural impulse response analysis, additional identification restrictions are required (e.g. that $C_{0}$ is lower-triangular). Note also that the restrictions such as $C_{0}$ being lower triangular are timing restrictions and must be thought about carefully. For instance, Bernanke, Boivin and Eliasz (2005) divide the elements of $y_{t}$ into blocks of "slow variables" (i.e. those which are slow to respond to a monetary policy shock) and "fast variables" as part of their identification scheme.

\subsection{The TVP-FAVAR}

In this monograph, we began by discussing Bayesian VAR modelling, before arguing that it might be desirable to allow the VAR coefficients to vary over time. This led us to the homoskedastic TVP-VAR. Next, we argued that it is usually empirically important to allow for multivariate stochastic volatility. We can go through exactly the same steps with the FAVAR resulting in a TVPFAVAR. Several specifications have been proposed, including Del Negro and Otrok (2008) and Korobilis (2009a). However, just as with TVP-VARs, it is 
worth stressing that TVP-FAVARs can be over-parameterized and that careful incorporation of prior information or the imposing of restrictions (e.g. only allowing some parameters to vary over time) can be important in obtaining sensible results. Completely unrestricted versions of them can be difficult to estimate using MCMC methods since they involve so many different state equations (i.e. one set for the factors and others to model the evolution of the parameters).

A very general specification for the TVP-FAVAR is given in Korobilis (2009a) ${ }^{32}$ who replaces (53) and (54) by

$$
y_{i t}=\lambda_{0 i t}+\lambda_{i t} f_{t}+\gamma_{i t} r_{t}+\varepsilon_{i t}
$$

and

$$
\left(\begin{array}{c}
f_{t} \\
r_{t}
\end{array}\right)=\widetilde{\Phi}_{1 t}\left(\begin{array}{c}
f_{t-1} \\
r_{t-1}
\end{array}\right)+. .+\widetilde{\Phi}_{p t}\left(\begin{array}{c}
f_{t-p} \\
r_{t-p}
\end{array}\right)+\widetilde{\varepsilon}_{t}^{f}
$$

and assumes each $\varepsilon_{i t}$ follows a univariate stochastic volatility process and $\operatorname{var}\left(\widetilde{\varepsilon}_{t}^{f}\right)=$ $\widetilde{\Sigma}_{t}^{f}$ has a multivariate stochastic volatility process of the form used in Primiceri (2005). Finally, the coefficients (for $i=1, . ., M) \lambda_{0 i t}, \lambda_{i t}, \gamma_{i t}, \widetilde{\Phi}_{1 t}, . ., \widetilde{\Phi}_{p t}$ are allowed to evolve according to random walks (i.e. state equations of the same form as 46 complete the model). All other assumptions are the same as for the FAVAR.

We will not describe the MCMC algorithm for this model other than to note that it simply adds more blocks to the MCMC algorithm for the FAVAR. These blocks are all of forms previously discussed in this monograph. For instance, the error variances in the measurement equations are drawn using the univariate stochastic volatility algorithm of Section 3.3.1. The algorithm of Section 3.3.2 can be used to draw $\widetilde{\Sigma}_{t}^{f}$. The coefficients $\lambda_{0 i t}, \lambda_{i t}, \gamma_{i t}, \widetilde{\Phi}_{1 t}, . ., \widetilde{\Phi}_{p t}$ are all drawn using the algorithm of Section 3.2, implemented in a very similar fashion as in the TVP-VAR. In short, as with so many models in empirical macroeconomics, Bayesian inference in the TVP-FAVAR proceeds by putting together an MCMC algorithm involving blocks from several simple and familiar algorithms.

\subsection{Empirical Illustration of Factor Methods}

To illustrate Bayesian inference in FAVARs and TVP-FAVAR models, we use a data set of 115 quarterly US macroeconomic variables spanning 1959Q1 though 2006Q3. Following common practice in this literature, we transform all variables to be stationary. For brevity, we do not list these variables here nor describe the required stationarity transformations. These details are provided in the manual on the website associated with this monograph.

The FAVAR is given in (53) and (54). It requires the choice of variables to isolate in $r_{t}$ and we use the same variables as in our previous VAR and

\footnotetext{
${ }^{32}$ The model of Korobilis (2009a) is actually a slight extension of this since it includes a dynamic mixture aspect similar to that presented in Section 4.1.3.
} 
TVP-VAR empirical illustrations: inflation, unemployment and the interest rate. Consequently, our FAVAR is the same tri-variate VAR used in previous empirical illustrations, augmented with factors, $f_{t}$, which are extracted from a large set of macroeconomic and financial variables.

We use principal components methods to extract the first two factors which are used in the FAVAR $(q=2)$ and two lags in the factor equation $(p=2) .{ }^{33}$ The use of principal components methods ensures identification of the model since it normalizes all factors to have mean zero and variance one. For the FAVAR we require a prior for the parameters $\Sigma^{f}, \Phi_{1}, . ., \Phi_{p}, \lambda_{0 i}, \lambda_{i}, \sigma_{i}^{2}$ for $i=1, . ., M$. Full details of this (relatively noninformative) prior are provided in the manual on the website associated with this monograph.

To carry out impulse response analysis we require additional identifying assumptions. With regards to the equations for $r_{t}$, we adopt the same identifying assumptions as in our previous empirical illustrations. These allow us to identify a monetary policy shock. With regards to the variables in $y_{t}$, suffice it to note here is that we adopt the same assumptions as Bernanke, Boivin and Eliasz (2005). The basic idea of their identifying scheme is described in Section 5.2.3 above.

Figures 6 and 7 plot impulse responses to the monetary policy shock for the FAVAR. Figure 6 plots impulse responses for the main variables which are included in $r_{t}$. The patterns in Figure 6 are broadly similar to those obtained in our empirical illustration using VARs (compare Figure 6 to Figure 4). However, the magnitudes of the impulse responses are somewhat different. Here we are finding more evidence that a monetary policy shock will decrease inflation. Figure 7 plots the response of a few randomly selected variables to the monetary policy shock. ${ }^{34}$

\footnotetext{
${ }^{33}$ These choices are only illustrative. In a substantive empirical exercise, the researcher would select these more carefully (e.g. using model selection methods such discussed previously in this monograph).

${ }^{34}$ See the manual on the website associated with this monograph for a definition of the abbreviations used in Figures 7 and 10. Briefly GDPC96 is GDP, GSAVE is savings, PRFI is private residential fixed investment, MANEMP is employment in manufacturing, AHEMAN is earnings in manufacturing, HOUST is housing starts, GS10 is a 10 year interest rate, EXJPUS is the Japanese-US exchange rate, PPIACO is a producer price index, OILPRICE is the oil price, HHSNTN is an index of consumer expectations and PMNO is the NAPM orders index. All impulse responses are to the original untransformed versions of these variables.
} 

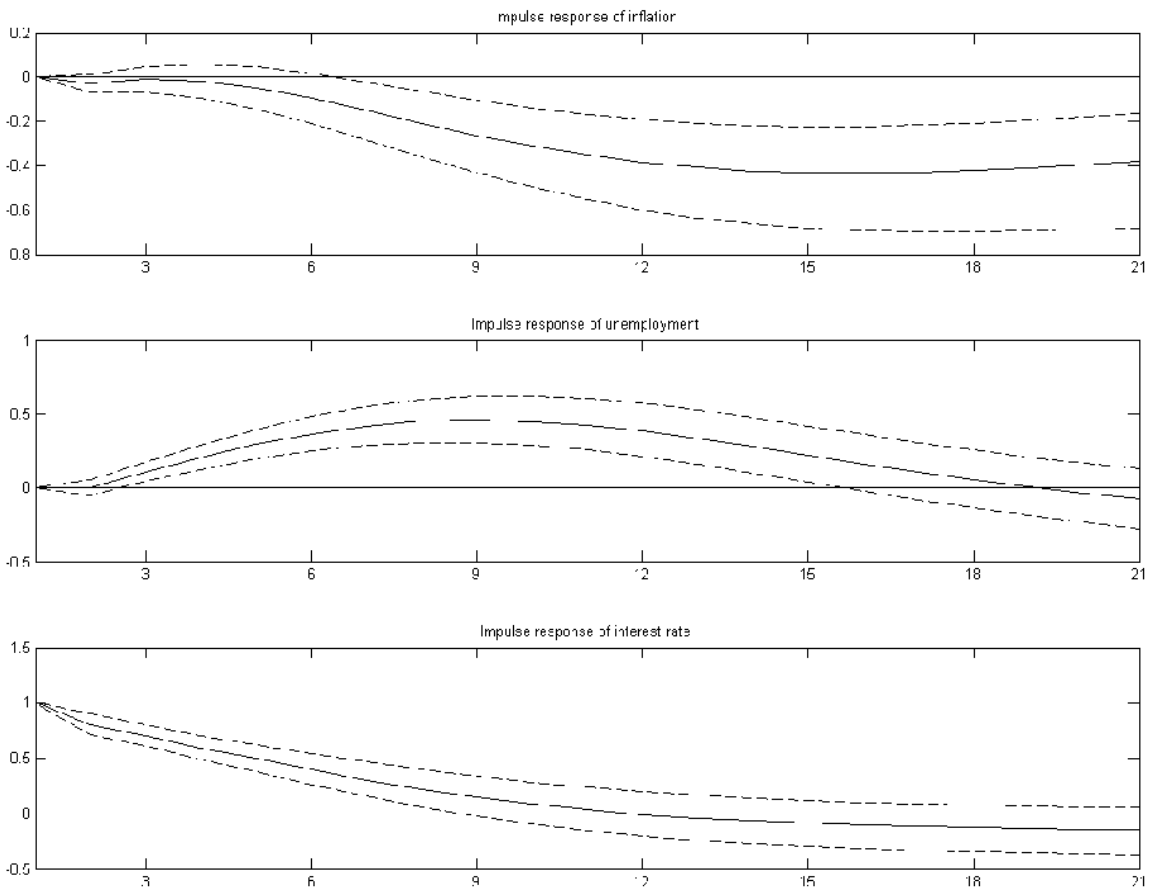

Figure 6: Posterior of impulse responses of main variables to monetary policy shock 

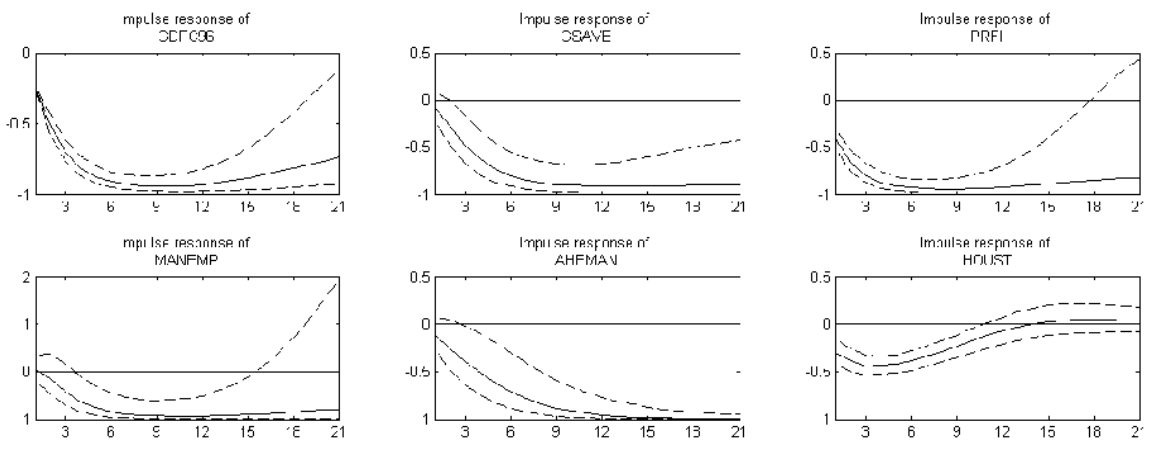

mpllse ressonse of

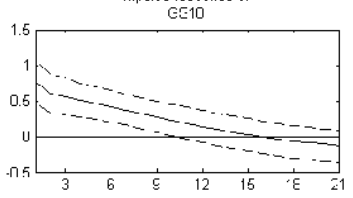

Inpu se reskonse o
[K.PUIS

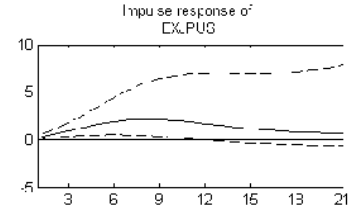

PPIACC
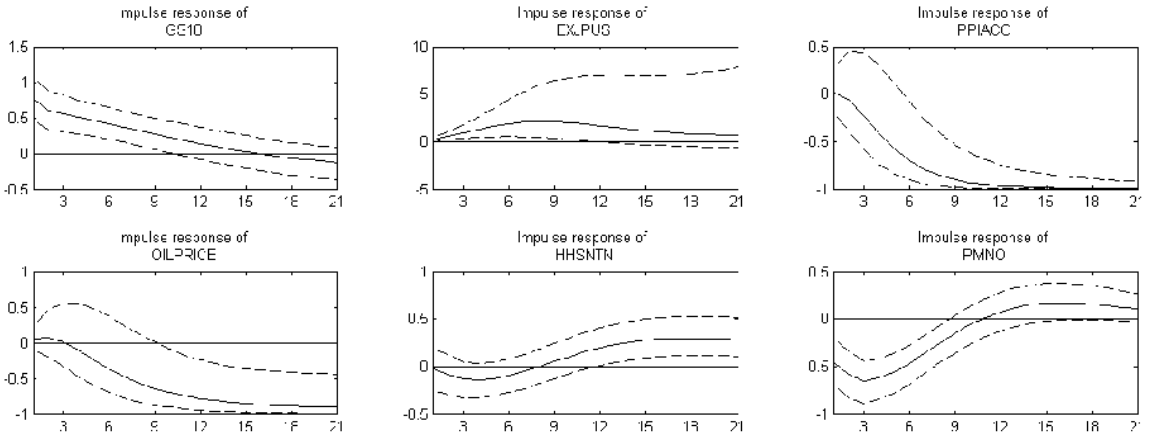

Imวulsa respoาs of
PMNO

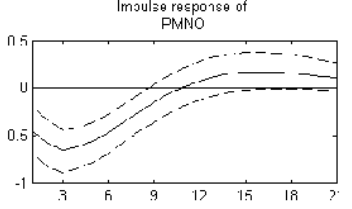

Figure 7: Posterior of impulse responses of selected variables to monetary policy shock

We now present results for a TVP-FAVAR defined in (55) and (56), but with the restriction that the parameters in the measurement equation are constant over time. ${ }^{35}$ The (relatively noninformative) prior we use is described in the manual on the website associated with this monograph.

Figure 8 plots the posterior means of the standard deviations of the errors in the two equations where the factors are the dependent variable and the three equations where $r_{t}$ are the dependent variables. It can be seen that there is substantive evidence of variation in volatilities (in particular, for equation for the first factor). The bottom three panels of Figure 8 look similar to Figure 5 and indicate the substantial increase in volatility associated with the 1970s and early 1980s preceding the Great Moderation of the business cycle. However, this pattern is somewhat muted relative to Figure 5 since the inclusion of the factors means that the standard deviation of the errors becomes smaller.

\footnotetext{
${ }^{35}$ We do this since it is difficult to estimate time variation in coefficients in both the measurement and state equation without additional restrictions or very strong prior information.
} 

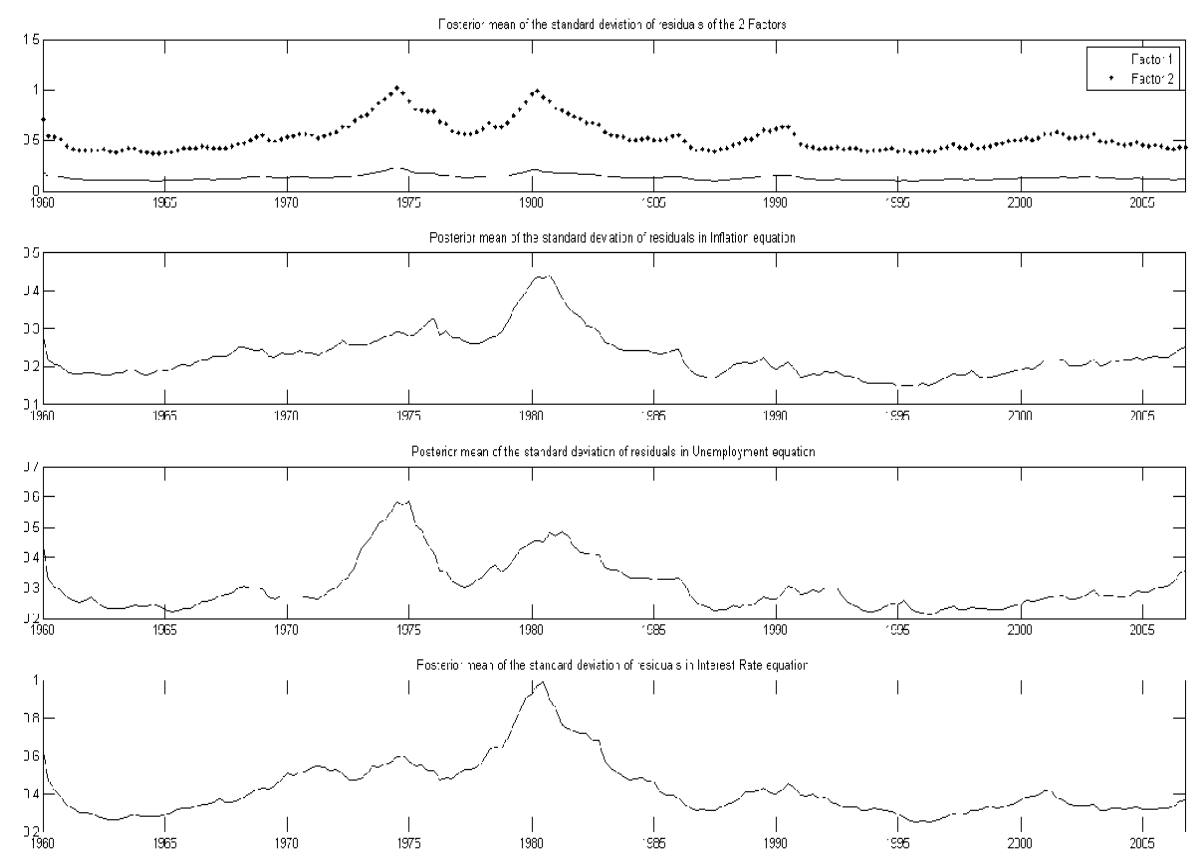

Figure 8: Time-varying volatilities of errors in five key equations of the TVP-FAVAR

Figures 9 and 10 plot impulse responses in the same format as Figures 6 and 7 . With the TVP-FAVAR these will be time varying so we plot them for three different time periods. A comparison of Figure 9 to Figure 4 (which presents the same impulse responses using a TVP-VAR) indicate that broad patterns are roughly similar, but there are some important differences between the two figures. Similarly, a comparison of Figure 10 to Figure 8 indicates broad similarities, but in some specific cases important differences can be found. 

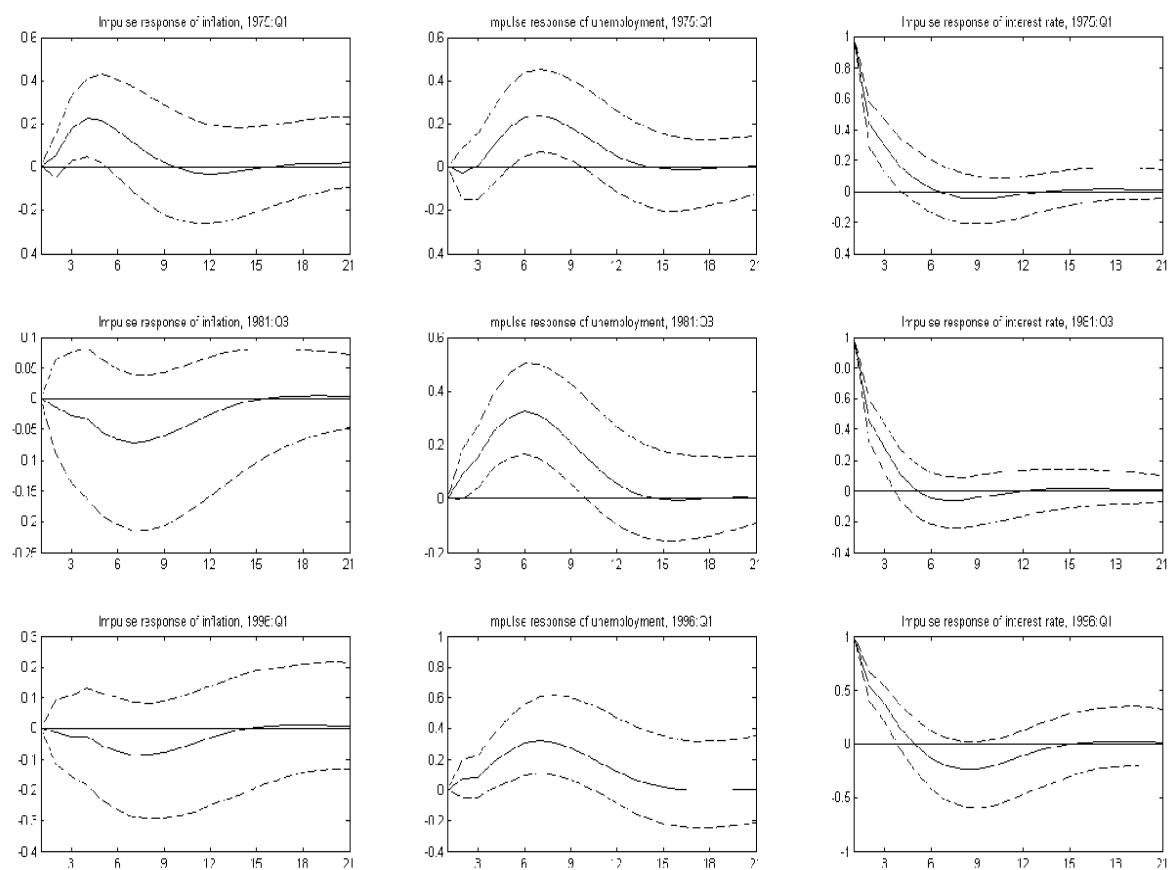

Figure 9: Posterior of impulse responses of main variables to monetary policy shock at different times 

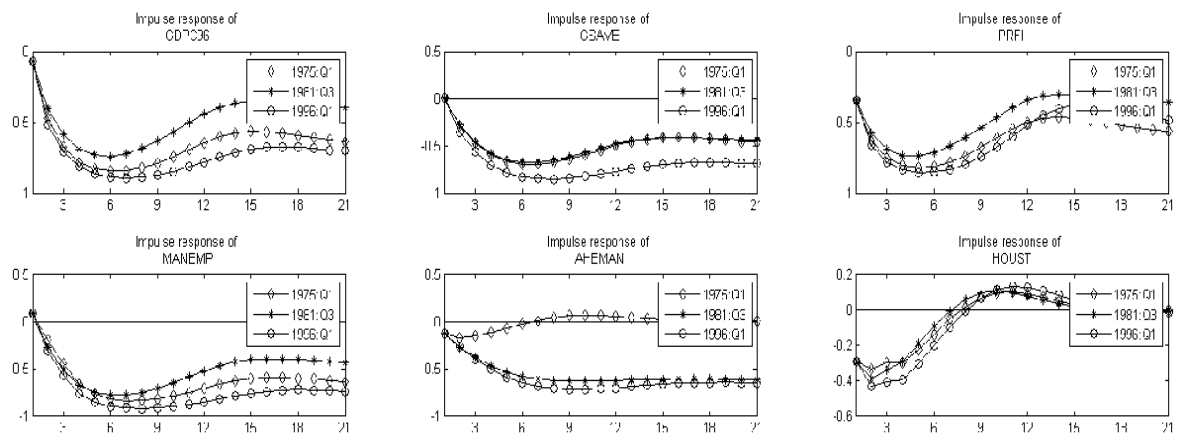

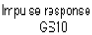

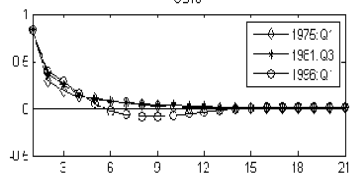

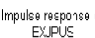

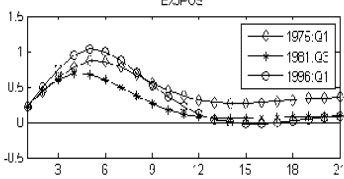

Impulsog ressonse
FFIACD

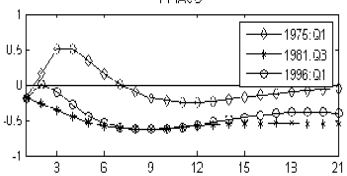

Inpuse issponse of
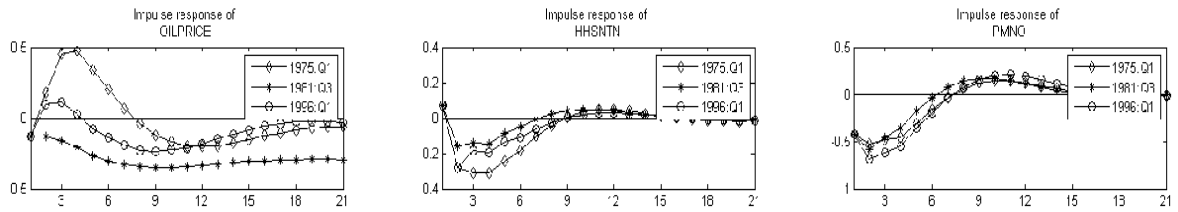

Figure 10: Posterior means impulse responses of selected variables to monetary policy shock at different times

\section{Conclusion}

In this monograph, we have discussed VARs, TVP-VAR, FAVARs and TVPFAVARs, including versions of these models with multivariate stochastic volatility. These classes of models have become very popular with empirical macroeconomists since they allow for insight into the relationships between macroeconomic variables in a manner which lets the data speak in a relatively unconstrained manner. However, the cost of working with such unconstrained models is that they risk being over-parameterized. Accordingly, researchers have found it desirable to impose soft or hard restrictions on these models. Soft restrictions typically involve shrinking coefficients towards a particular value (usually zero) whereas hard ones involve the imposition of exact restrictions. Bayesian methods have been found to be an attractive and logically consistent way of handling such restrictions.

We have shown have Bayesian inference can be implemented in a variety of ways in these models, with emphasis on specifications that are of interest to the practitioner. Apart from the simplest of VARs, MCMC algorithms are required. This monograph describes these algorithms in a varying degree of detail. We 
draw the reader's attention to the website associated with this monograph which contains Matlab code for most of the models described above. A manual on this website provides a complete listing of all formulae used in each MCMC algorithm. Thus, our aim has been to provide a complete set of Bayesian tools for the practitioner interested in a wide variety of models commonly used in empirical macroeconomics. 


\section{References}

An, S. and Schorfheide, F. (2007). "Bayesian analysis of DSGE models," Econometric Reviews, 26, 113-172.

Asai, M., McAleer, M. and Yu, J. (2006). "Multivariate stochastic volatility: A review," Econometric Reviews, 25, 145-175.

Ballabriga, F., Sebastian, M. and Valles, J. (1999). "European asymmetries," Journal of International Economics, 48, 233-253.

Banbura, M., Giannone, D. and Reichlin, L. (2010). "Large Bayesian VARs," Journal of Applied Econometrics, 25, 71-92.

Belviso, F. and Milani, F. (2006). "Structural factor augmented VARs (SFAVARs) and the effects of monetary policy," Topics in Macroeconomics, $6,2$.

Berg, A., Meyer, R. and Yu, J. (2004). "Deviance information criterion for comparing stochastic volatility models," Journal of Business and Economic Statistics, 22, 107-120.

Bernanke, B. and Boivin, J. (2003). "Monetary policy in a data-rich environment," Journal of Monetary Economics, 50, 525-546.

Bernanke, B., Boivin, J. and Eliasz, P. (2005). "Measuring monetary policy: A Factor augmented vector autoregressive (FAVAR) approach," Quarterly Journal of Economics, 120, 387-422.

Bernanke, B. and Mihov, I. (1998). "Measuring monetary policy," Quarterly Journal of Economics, 113, 869-902.

Boivin, J. and Giannoni, M. (2006). "Has monetary policy become more effective?" Review of Economics and Statistics, 88, 445-462.

Canova, F. (2007). Methods for Applied Macroeconomic Research published by Princeton University Press.

Canova, F. (1993). "Modelling and forecasting exchange rates using a Bayesian time varying coefficient model," Journal of Economic Dynamics and Control, 17, 233-262.

Canova, F. and Ciccarelli, M. (2009). "Estimating multi-country VAR models," International Economic Review, 50, 929-959.

Canova, F. and Ciccarelli, M. (2004). "Forecasting and turning point predictions in a Bayesian panel VAR model," Journal of Econometrics, 120, 327-359.

Canova, F. and Gambetti, L. (2009). "Structural changes in the US economy: Is there a role for monetary policy?" Journal of Economic Dynamics and Control, 33, 477-490.

Carlin, B. and Chib, S. (1995). "Bayesian model choice via Markov chain Monte Carlo methods," Journal of the Royal Statistical Society, Series B, 57, 473-84.

Carter, C. and Kohn, R. (1994). "On Gibbs sampling for state space models," Biometrika, 81, 541-553.

Chan, J.C.C. and Jeliazkov, I. (2009). "Efficient simulation and integrated likelihood estimation in state space models," International Journal of Mathematical Modelling and Numerical Optimisation, 1, 101-120.

Chan, J.C.C., Koop, G., Leon-Gonzalez, R. and Strachan, R. (2010). "Time varying dimension models," manuscript available at http://personal.strath.ac.uk/gary.koop/. 
Chib, S. and Greenberg, E. (1994). "Bayes inference in regression models with ARMA(p,q) errors," Journal of Econometrics, 64, 183-206.

Chib, S. and Greenberg, E. (1995). "Hierarchical analysis of SUR models with extensions to correlated serial errors and time-varying parameter models," Journal of Econometrics, 68, 339-360.

Chib, S. and Jeliazkov, I. (2001). "Marginal likelihood from the MetropolisHastings output," Journal of the American Statistical Association, 96, 270-281.

Chib, S. and Jeliazkov, I. (2005). "Accept-reject Metropolis-Hastings sampling and marginal likelihood estimation," Statistica Neerlandica, 59, 30-44.

Chib, S., Nardari, F. and Shephard, N. (2002). "Markov chain Monte Carlo methods for stochastic volatility models," Journal of Econometrics, 108, 281316.

Chib, S., Nardari, F. and Shephard, N. (2006). "Analysis of high dimensional multivariate stochastic volatility models," Journal of Econometrics, 134, 341371.

Chib, S., Omori, Y. and Asai, M. (2009). "Multivariate stochastic volatility," pages 365-400 in Handbook of Financial Time Series, edited by T. Andersen, R. David, J. Kreiss and T. Mikosch. Berlin: Springer-Verlag.

Chib, S. and Ramamurthy, S. (2010). "Tailored Randomized Block MCMC Methods with Application to DSGE Models," Journal of Econometrics, 155, 19-38.

Chipman, H., George, E. and McCulloch, R. (2001). "Practical implementation of Bayesian model selection," pages 67-116 in Model Selection, edited by P. Lahiri. Volume 38, IMS Lecture Notes.

Christiano, L., Eichenbaum, M. and Evans, C. (1999). "Monetary shocks: What have we learned and to what end?", pages 65-148 in Handbook of Macroeconomics, vol. 1A, edited by J. Taylor and M. Woodford. New York: Elsevier.

Ciccarelli, M. and Rebucci, A., (2002). "The transmission mechanism of European monetary policy: Is there heterogeneity? Is it changing over time?," International Monetary Fund working paper, WP 02/54.

Cogley, T. and Sargent, T. (2001). "Evolving post-World War II inflation dynamics," NBER Macroeconomic Annual, 16, 331-373.

Cogley, T. and Sargent, T. (2005). "Drifts and volatilities: Monetary policies and outcomes in the post WWII U.S," Review of Economic Dynamics, 8, 262302.

DeJong, P. and Shephard, N. (1995). "The simulation smoother for time series models," Biometrika, 82, 339-350.

Del Negro, M. and Otrok, C. (2008). "Dynamic factor models with time varying parameters: Measuring changes in international business cycles," Federal Reserve Bank of New York Staff Report no. 326.

Del Negro, M. and Schorfheide, F. (2004). "Priors from general equilibrium models for VARs," International Economic Review, 45, 643-673.

Del Negro, M. and Schorfheide, F. (2010). "Bayesian macroeconometrics," to appear in the Handbook of Bayesian Econometrics, edited by J. Geweke, G. Koop and H. van Dijk. Oxford: Oxford University Press. 
Doan, T., Litterman, R. and Sims, C. (1984). "Forecasting and conditional projection using realistic prior distributions," Econometric Reviews, 3, 1-144.

Doucet, A., Godsill, S. and Andrieu, C. (2000). "On sequential Monte Carlo sampling methods for Bayesian filtering," Statistics and Computing, 10, 197-208.

Durbin, J. and Koopman, S. (2001). Time Series Analysis by State Space Methods. Oxford: Oxford University Press.

Durbin, J. and Koopman, S. (2002). "A simple and efficient simulation smoother for state space time series analysis," Biometrika, 89, 603-616.

Fernandes-Villaverde, J. (2009). "The econometrics of DSGE models," Penn Institute for Economic Research working paper 09-008.

Fernandez, C., Ley, E. and Steel, M. (2001). "Benchmark priors for Bayesian model averaging," Journal of Econometrics, 100, 381-427.

Forni, M. and Reichlin, L. (1998). "Let's get real: A factor analytic approach to disaggregated business cycle dynamics," Review of Economic Studies, 65, 453473.

Fruhwirth-Schnatter, S. (1994). "Data augmentation and dynamic linear models," Journal of Time Series Analysis, 15, 183-202.

Fruhwirth-Schnatter, S. and Wagner, H. (2008). "Marginal likelihoods for non-Gaussian models using auxiliary mixture sampling," Computational Statistics and Data Analysis, 52, 4608-4624.

Gelfand, A. and Dey, D. (1994). "Bayesian model choice: Asymptotics and exact calculations," Journal of the Royal Statistical Society Series B, 56, 501514.

George, E., Sun, D. and Ni, S. (2008). "Bayesian stochastic search for VAR model restrictions," Journal of Econometrics, 142, 553-580.

Geweke, J. (1977). "The dynamic factor analysis of economic time series," in Latent Variables in Socio-economic Models, edited by D. Aigner and A. Goldberger, Amsterdam: North Holland.

Geweke, J. (1996). "Bayesian reduced rank regression in econometrics," Journal of Econometrics, 75, 121-146.

Geweke, J. and Amisano, J. (2010). "Hierarchical Markov normal mixture models with applications to financial asset returns," Journal of Applied Econometrics, forthcoming.

Geweke, J. and Keane, M. (2007). "Smoothly mixing regressions," Journal of Econometrics, 138, 252-291.

Geweke, J. and Zhou, G. (1996). "Measuring the pricing error of the arbitrage pricing theory," Review of Financial Studies, 9, 557-587.

Giordani, P. and Kohn, R. (2008). "Efficient Bayesian inference for multiple change-point and mixture innovation models," Journal of Business and Economic Statistics, 26, 66-77.

Giordani, P., Pitt, M. and Kohn, R. (2010). "Time series state space models," to appear in the Handbook of Bayesian Econometrics, edited by J. Geweke, G. Koop and H. van Dijk. Oxford: Oxford University Press.

Giordani, P., Kohn, R. and van Dijk, D. (2007). "A unified approach to nonlinearity, structural change and outliers," Journal of Econometrics, 137, 112133. 
Green, P. (1995). "Reversible jump Markov chain Monte Carlo computation and Bayesian model determination," Biometrika, 82, 711-32.

Groen, J., Paap, R. and Ravazzolo, F. (2008). "Real-time inflation forecasting in a changing world," Erasmus University manuscript, 2008.

Harvey, A. (1989). Forecasting, Structural Time Series Models and the Kalman Filter, Cambridge University Press: Cambridge.

Ingram, B. and Whiteman, C. (1994). "Supplanting the Minnesota prior Forecasting macroeconomic time series using real business cycle model priors," Journal of Monetary Economics, 49, 1131-1159.

Jacquier, E., Polson, N. and Rossi, P. (1994). "Bayesian analysis of stochastic volatility," Journal of Business and Economic Statistics, 12, 371-417.

Johannes, M. and Polson, N. (2009). "Particle filtering," pages 1015-1030 in Handbook of Financial Time Series, edited by T. Andersen, R. David, J. Kreiss and T. Mikosch. Berlin: Springer-Verlag.

Kadiyala, K. and Karlsson, S. (1997). "Numerical methods for estimation and inference in Bayesian VAR models," Journal of Applied Econometrics, 12, 99-132.

Kim, C. and Nelson, C. (1999). State Space Models with Regime Switching. Cambridge: MIT Press.

Kim, S., Shephard, N. and Chib, S. (1998). Stochastic volatility: likelihood inference and comparison with ARCH models, Review of Economic Studies, 65, 361-93.

Koop, G. (1992). "Aggregate shocks and macroeconomic fluctuations: A Bayesian approach," Journal of Applied Econometrics, 7, 395-411.

Koop, G. (1996). "Parameter uncertainty and impulse response analysis," Journal of Econometrics, 72, 135-149.

Koop, G. (2010). "Forecasting with medium and large Bayesian VARs," manuscript available at http://personal.strath.ac.uk/gary.koop/.

Koop, G., Leon-Gonzalez, R. and Strachan, R. (2009). "On the evolution of the monetary policy transmission mechanism," Journal of Economic Dynamics and Control, 33, 997-1017.

Koop, G., Poirier, D. and Tobias, J. (2007). Bayesian Econometric Methods. Cambridge: Cambridge University Press.

Koop, G. and Potter, S. (2004). "Forecasting in dynamic factor models using Bayesian model averaging," The Econometrics Journal, 7, 550-565.

Koop, G. and Potter, S. (2006). "The Vector floor and ceiling model," Chapter 4 in Nonlinear Time Series Analysis of the Business Cycle, edited by C. Milas, P. Rothman and D. van Dijk in Elsevier's Contributions to Economic Analysis series.

Koop, G. and Potter, S. (2009). "Time varying VARs with inequality restrictions," manuscript available at http://personal.strath.ac.uk/gary.koop/koop_potter14.pdf.

Korobilis, D. (2009a). "Assessing the transmission of monetary policy shocks using dynamic factor models," University of Strathclyde, Discussion Papers in Economics, No. 09-14.

Korobilis, D. (2009b). "VAR forecasting using Bayesian variable selection," manuscript. 
Kose, A., Otrok, C. and Whiteman, C. (2003). "International business cycles: World, region and country-specific factors," American Economic Review, 93, 1216-1239.

Kuo, L. and B. Mallick. (1997). "Variable selection for regression models," Shankya: The Indian Journal of Statistics (Series B), 60, 65-81.

Litterman, R. (1986). "Forecasting with Bayesian vector autoregressions Five years of experience," Journal of Business and Economic Statistics, 4, 25-38.

Lopes, H. and West, M. (2004). "Bayesian model assessment in factor analysis," Statistica Sinica, 14, 41-67.

Lubik, T. and Schorfheide, F. (2004). "Testing for indeterminacy: An application to U.S. monetary policy," American Economic Review, 94, 190-217.

McCausland, W., Miller, S. and Pelletier, D. (2007). "A new approach to drawing states in state space models," working paper 2007-06, Université de Montréal, Département de sciences économiques.

Omori, Y., Chib, S., Shephard, N. and Nakajima, J. (2007). "Stochastic volatility with leverage: Fast and efficient likelihood inference," Journal of Econometrics, 140, 425-449.

Otrok, C. and Whiteman, C. (1998). "Bayesian leading indicators: Measuring and predicting economic conditions in Iowa," International Economic Review, 39, 997-1014.

Paap, R. and van Dijk, H. (2003). "Bayes estimates of Markov trends in possibly cointegrated series: An application to US consumption and income," Journal of Business and Economic Statistics, 21, 547-563.

Pitt, M. and Shephard, N. (1999). "Time varying covariances: a factor stochastic volatility approach," pages 547-570 in Bayesian Statistics, Volume 6, edited by J. Bernardo, J.O. Berger, A.P. Dawid and A.F.M. Smith, Oxford: Oxford University Press.

Primiceri. G., (2005). "Time varying structural vector autoregressions and monetary policy," Review of Economic Studies, 72, 821-852.

Rubio-Ramirez, J., Waggoner, D. and Zha, T. (2010). "Structural vector autoregressions: Theory of identification and algorithms for inference," Review of Economic Studies, forthcoming.

Sentana, E. and Fiorentini, G. (2001). "Identification, estimation and testing of conditionally heteroskedastic factor models," Journal of Econometrics, 102, 143-164.

Sims, C. (1993). "A nine variable probabilistic macroeconomic forecasting model," pp. 179-204 in J. Stock and M. Watson, eds., Business Cycles Indicators and Forecasting. University of Chicago Press for the NBER).

Sims, C. (1980). "Macroeconomics and reality," Econometrica, 48, 1-48.

Sims, C. and Zha, T. (1998). "Bayesian methods for dynamic multivariate models," International Economic Review, 39, 949-968.

Sims, C. and Zha, T. (2006). "Were there regime switches in macroeconomic policy?" American Economic Review, 96, 54-81.

Stock, J. and Watson, M., (1996). "Evidence on Structural Instability in Macroeconomic Time Series Relations," Journal of Business and Economic Statistics, 14, 11-30. 
Stock, J. and Watson, M., (1999). "Forecasting inflation," Journal of Monetary Economics, 44, 293-335.

Stock, J. and Watson, M., (2002). "Macroeconomic forecasting using diffusion indexes," Journal of Business and Economic Statistics, 20, 147-162.

Stock, J. and Watson, M., (2005). "Implications of dynamic factor models for VAR analysis," National Bureau of Economic Research working paper 11467.

Stock, J. and Watson, M., (2006). "Forecasting using many predictors," pp. 515-554 in Handbook of Economic Forecasting, Volume 1, edited by G. Elliott, C. Granger and A. Timmerman, Amsterdam: North Holland.

Stock, J. and Watson, M., (2008). "Forecasting in dynamic factor models subject to structural instability," in The Methodology and Practice of Econometrics, A Festschrift in Honour of Professor David F. Hendry, edited by J. Castle and N. Shephard, Oxford: Oxford University Press.

Villani, M. (2009). "Steady-state prior for vector autoregressions," Journal of Applied Econometrics, 24, 630-650.

West, M. (2003). "Bayesian factor regression models in the 'large p, small n' paradigm," pages 723-732 in Bayesian Statistics, Volume 7, edited by J.M. Bernardo, M. Bayarri, J.O. Berger, A.P. Dawid, D. Heckerman, A.F.M. Smith, and M. West, Oxford: Oxford University Press.

West, M. and Harrison, P. (1997). Bayesian Forecasting and Dynamic Models. Second edition. Berlin: Springer. 